\title{
Selective transfer of exosomes from oligodendrocytes to microglia by macropinocytosis
}

\author{
Doctoral Thesis \\ In partial fulfillment of the requirements \\ for the degree 'Doctor of rerum naturalium (Dr. rer. nat.)' \\ in the Molecular Medicine Study Program \\ at the Georg August University Göttingen, \\ Faculty of Medicine
}

submitted by

Mareike Schnaars

born in

Bremen, Germany

Göttingen 2010 


\section{Thesis Committee:}

Supervisor:

Prof. Mikael Simons, Zelluläre Neurobiologie

Max-Planck-Institut für Experimentelle Medizin, Göttingen

Second member of the thesis committee:

Prof. Uwe-Karsten Hanisch, Abteilung Neuropathologie

Universitätsmedizin Göttingen, Georg-August-Universität

Third member of the thesis committee:

Prof. Jürgen Wienands, Abteilung Zelluläre und Molekulare Immunologie Universitätsmedizin Göttingen, Georg-August-Universität

\section{Date of Disputation:}




\section{Affidavit}

I hereby declare that this Doctoral thesis entitled 'Selective transfer of exosomes from oligodendrocytes to microglia by macropinocytosis' has been written independently with no other aids or sources than quoted. This thesis (wholly or in part) has not been submitted elsewhere for any academic award or qualification.

Mareike Schnaars

December 2010

Göttingen, Germany 


\section{Related publications}

The results of this project are communicated in the following publication. I am grateful to all co-authors for the stimulating and fruitful collaborations.

- Dirk Fitzner*, Mareike Schnaars*\#, Denise van Rossum, Gurumoorthy Krishnamoorthy, Payam Dibaj, Mostafa Bakhti, Tommy Regen, Uwe-Karsten Hanisch, Mikael Simons" . 'Selective transfer of exosomes from oligodendrocytes to microglia by macropinocytosis'. Journal of Cell Science, accepted *equal contribution to first authorship

\# corresponding authors

Parts of the work described in this thesis have been presented at international meetings and symposia. I want to sincerely thank all people who contributed to these studies.

- Mareike Schnaars*, Dirk Fitzner*, Denise van Rossum, Gurumoorthy Krishnamoorthy, Payam Dibaj, Tommy Regen, Uwe-Karsten Hanisch, Mikael Simons. 'Selective transfer of exosomes from oligodendrocytes to microglia', Glia in Health and Disease 2010, Cold Spring Habour NY, USA, oral presentation

- Mareike Schnaars*, Dirk Fitzner*, Denise van Rossum, Gurumoorthy Krishnamoorthy, Payam Dibaj, Tommy Regen, Uwe-Karsten Hanisch, Mikael Simons. 'Selective transfer of vesicles containing encephalitogenic antigens from oligodendrocytes to microglia', Protein Trafficking in Health and Disease 2010, Hamburg, Germany, Poster presentation awarded 'Poster Prize 2010' 
- Mareike Schnaars*, Dirk Fitzner*, Denise van Rossum, Tommy Regen, UweKarsten Hanisch, Mikael Simons. 'Clearance of oligodendrocyte-derived exosomes by microglia via macropinocytosis', Neurizons 2010, Göttingen, Germany, Poster presentation 


\section{Contents}

Contents vi

List of Figures . . . . . . . . . . . . . . . . . . . xi

List of Tables . . . . . . . . . . . . . . . . . . . . . xiii

Abbreviations $\quad$ xiv

Acknowledgements $\quad$ xviii

Abstract $\quad$ xx

1 Introduction $\quad 1$

1.1 Exosomes .......................... . . 1

1.1.1 Formation of exosomes . . . . . . . . . . . . . 2

1.1.2 Lipid and protein composition of exosomes . . . . . . . . . 3

1.1.3 Release of exosomes . . . . . . . . . . . . . . . . . . 5

1.1.4 Function of exosomes . . . . . . . . . . . . . . . . 6

Pathway of protein and lipid disposal . . . . . . . . . . . 6

Intercellular communication . . . . . . . . . . . . . . 7

Exosomes in vivo and their application in diagnostics . . . . . 8

1.2 Glia cells in the central nervous system . . . . . . . . . . . . . . . . . 10

1.2.1 Oligodendrocytes . . . . . . . . . . . . . . . . . 11

Composition of the myelin membrane . . . . . . . . . . 11

Origin of oligodendrocytes . . . . . . . . . . . . . . 12

Oligodendroglial exosomes . . . . . . . . . . . . . . 12

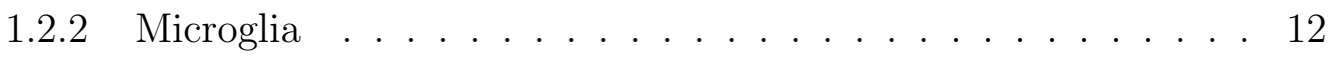

1.2.3 Innate immunity mediated by microglia . . . . . . . . . . . 13 
1.2.4 Antigen presentation by microglia . . . . . . . . . . . 15

Antigen-presenting cells . . . . . . . . . . . . . 15

Major histocompatibility complexes . . . . . . . . . 16

Antigen presentation by microglia . . . . . . . . . . . 17

Chemokines and microglia . . . . . . . . . . . 18

Cytokines and microglia . . . . . . . . . . . . . . . 19

1.2.5 Microglia and multiple sclerosis . . . . . . . . . . . . . 20

1.3 Endocytic pathways in mammalian cells . . . . . . . . . . . . . 22

1.3.1 Phagocytosis . . . . . . . . . . . . . . 23

1.3.2 Macropinocytosis .................. 24

1.4 Aims and objectives . . . . . . . . . . . . . . . . 25

2 Materials \& methods $\quad 26$

2.1 Materials ........................ 26

2.1.1 Chemicals and consumables . . . . . . . . . 26

2.1.2 Antibodies and fluorophore-coupled compounds . . . . . . . 26

2.1.3 DNA constructs . . . . . . . . . . . . . . . . . 27

2.1.4 Buffers and solutions . . . . . . . . . . . . . . . 28

2.1.5 Cell culture media . . . . . . . . . . . . . . . . . . 28

Super SATO Medium . . . . . . . . . . . . . . . . . . 28

SATO Medium .................. . 29

2.1.6 Inhibitors, sugars and peptides for cell culture assays . . . . . 29

2.1.7 Commercial kits . . . . . . . . . . . . . . . . . . . . . . . 29

2.1 .8 Specific software . . . . . . . . . . . . . . . . . 29

2.2 Methods . . . . . . . . . . . . . . . . . . 30

2.2.1 Biochemistry . . . . . . . . . . . . . 30

Preparation of cell lysates . . . . . . . . . . . . . 30

Determination of protein concentration . . . . . . . . . 31

Sodium dodecyl sulfate polyacrylamide gel electrophoresis (SDSPAGE) . . . . . . . . . . . . . . . 32

Western blotting .................. . . 33

2.2.2 Cell culture techniques . . . . . . . . . . . . . . . . . . 34 
Primary glial culture . . . . . . . . . . . . . . . 34

Primary neuronal culture . . . . . . . . . . . . . . . 35

Growth and maintenance of EOC-20 cell line . . . . . . . . . 35

Growth and maintenance of L929 cell line . . . . . . . . . . 36

Growth and maintenance of Oli-neu cell line . . . . . . . . . 36

2.2.3 Transfection of mammalian cells . . . . . . . . . . . . 36

2.2.4 Exosome purification and labeling . . . . . . . . . . . 37

Exosome purification . . . . . . . . . . . . . 37

Analysis of exosomes on continuous sucrose gradients . . . . . 37

Fluorescent labeling of exosomes . . . . . . . . . . . . . 38

2.2.5 Exosome internalization assay . . . . . . . . . . . . 38

In vitro exosome uptake assay . . . . . . . . . . . . . . . . 38

Liposome preparation . . . . . . . . . . . . . . . . . 39

Live imaging of exosome internalization . . . . . . . . . . 39

2.2.6 $\mathrm{S}^{35}$ Metabolic labeling of proteins . . . . . . . . . . . . . . 40

2.2.7 Myelin preparation and internalization assay . . . . . . . . 40

Myelin purification on discontinuous sucrose gradients . . . . . 41

Fluorescent labeling of myelin . . . . . . . . . . . . . 41

Myelin internalization assay . . . . . . . . . . . 41

2.2.8 Cytokine release assays . . . . . . . . . . . . . . . . . 42

2.2.9 Flow cytometric measurements . . . . . . . . . . . . . 43

Flow cytometric analysis of primary microglia . . . . . . . 43

Flow cytometric analysis of exosomes . . . . . . . . . . 43

2.2.10 T cell activation assay . . . . . . . . . . . . . . . . . 44

2.2.11 Stereotactic injection into the murine brain . . . . . . . . . . 44

Injection of IFN $\gamma$ into the hippocampus . . . . . . . . . . 44

Cryosectioning of mouse brains . . . . . . . . . . . . 45

2.2 .12 Immunohistochemistry . . . . . . . . . . . . . . . 45

2.2.13 Immunocytochemistry . . . . . . . . . . . . . 46

2.2.14 Microscopy and image processing . . . . . . . . . . . 47

Two photon in vivo imaging of microglia . . . . . . . . . 47

Confocal microscopy . . . . . . . . . . . . . . . . . . . . . . . 48 


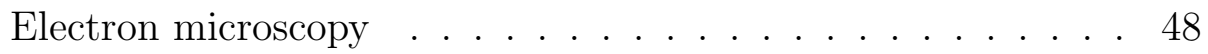

$\begin{array}{lll}3 & \text { Results } & 49\end{array}$

3.1 Exosomes are released by oligodendrocytes . . . . . . . . . . . . . 49

3.1.1 Isolation and characterization of oligodendroglial exosomes . . 49

Sucrose gradient analysis of the $100,000 \times$ g pellet . . . . . . 50

3.1.2 Quantitative analysis of oligodendroglial exosome release . . . 51

3.2 Exosomes are specifically internalized by microglia . . . . . . . . . . . 52

3.2.1 Internalization of oligodendrocyte-derived exosomes by microglia . . . . . . . . . . . . . . . . 5 52

3.2.2 Exosomes are internalized by microglia in vivo . . . . . . . . . 60

3.3 Microglia internalize exosomes by macropinocytosis . . . . . . . . . . 62

3.3.1 Which endocytic pathway conveys exosome internalization by microglia? . . . . . . . . . . . . . . . . 62

3.3.2 Is the internalization of exosomes receptor dependent? . . . . 65

3.3.3 Uptake of exosomes is reduced in the presence of phosphatidylserine containing liposomes . . . . . . . . . . . . . 66

3.3.4 Exosome internalization is increased in presence of recombinant milk-fat globulin E 8 . . . . . . . . . . . . . . . 68

3.3.5 Fluid phase uptake versus phagocytosis - Internalization of dextran and myelin . . . . . . . . . . . . . 70

3.4 Exosome uptake occurs in an immunologically silent manner. . . . . . 75

3.5 Inflammatory stimuli downregulate macropinocytosis of exosomes in microglia . . . . . . . . . . . . . . . . 80

3.6 Functional heterogeneity in microglial population of the CNS? . . . . 84

$\begin{array}{llr}4 & \text { Discussion } & 87\end{array}$

4.1 Exosomes are selectively internalized by microglia - in vitro and in vivo 87

4.1.1 Which key players of endocytic mechanisms are involved in exosome internalization? . . . . . . . . . . . . . . . 88

4.1.2 Is oligodendroglial exosome internalization a receptor mediated process? . . . . . . . . . . . . . . . . . . . 90 
Phosphatidylserine - a link to specific microglial receptors for exosome internalization? . . . . . . . . . . . . . 91

4.1.3 Oligodendroglial exosomes are cleared by macropinocytosis . . 92

4.2 Exosome internalization by microglia: an immunologically silent process? . . . . . . . . . . . . . . . . . . . . 93

4.2.1 Cytokine release by microglia after exosome internalization . . 93

4.3 Is exosomal antigen presented by microglia? . . . . . . . . . . . . . . 94

Cellular responses to $\mathrm{IFN}_{\gamma} \ldots \ldots$. . . . . . . . . . . 94

4.3.1 Macropinocytosis and antigen presentation by microglia . . . . 95

Processing of exosomal antigens for presentation on $\mathrm{MHC}$ molecules?. . . . . . . . . . . . . . 96

Exosomal lipids as putative antigens for presentation by microglia? . . . . . . . . . . . . . 97

4.3.2 Regulation of macropinocytosis and antigen presentation by microglia . . . . . . . . . . . . . . . . . . 98

4.4 Functional heterogeneity of microglia . . . . . . . . . . . . . . . 99

4.4.1 Microglia vs. macrophages . . . . . . . . . . . . . . . 99

4.4.2 Microglial heterogeneity - cellular microenvironment or intrinsic cues? . . . . . . . . . . . . . . . . . . . . 101

4.5 Function of oligodendroglial exosomes . . . . . . . . . . . . . . . 102

4.5.1 Exosomes and autoimmunity? . . . . . . . . . . . . 103

Exosomes - shuttle of infectious agents and autoantigens? . . . 104

$\begin{array}{llr}5 & \text { Summary and conclusions } & 106\end{array}$

$\begin{array}{ll}\text { Bibliography } & 108\end{array}$

$\begin{array}{lr}\text { Curriculum Vitae } & 140\end{array}$ 


\section{List of Figures}

1.1 Multivesicular bodies fuse with the plasma membrane to release exosomes . . . . . . . . . . . . . . . . . . 3

1.2 Representative protein composition of exosomes . . . . . . . . . . 4

1.3 Microglial cell in the hippocampal area of the adult murine brain . . 14

1.4 Endocytic pathways in mammalian cells . . . . . . . . . . . . . . 22

3.1 Exosomes of rat primary oligodendrocytes contain exosomal marker and major myelin proteins . . . . . . . . . . . . . . 50 50

3.2 Exosomes of the oligodendroglial cell line Oli-neu contain typical exosomal marker proteins . . . . . . . . . . . . . . . . . . . 51

3.3 Quantitative analysis of exosome release by oligodendrocytes . . . . 52

$3.4 \quad$ Oligodendroglial exosomes are selectively internalized by microglia. 53

3.5 Oligodendroglial-derived exosomes are transferred to the late endosomes $/$ lysosomes . . . . . . . . . . . . . . . 54

3.6 Exosomes of rat primary oligodendrocytes contain exosomal marker and mayor myelin proteins . . . . . . . . . . . . . . 55

3.7 The lipophilic dye PKH67 does not diffuse into the plasma membrane of microglia . . . . . . . . . . . . . . . . . . 56

3.8 Internalization pattern of sucrose-gradient purified vesicles in microglia is identical to exosomes . . . . . . . . . . . . . . . . 57

3.9 Exosomes are cleared by microglia but not by astrocytes . . . . . . 58

3.10 Exosomes are internalized by microglia in vivo. . . . . . . . . . . . . 61

3.11 Macropinocytosis of exosomes, visualized by live cell imaging of microglial EOC cells . . . . . . . . . . . . . . . . . 63

3.12 Microglia internalize exosomes via macropinocytosis . . . . . . . . . 64

3.13 Microglia internalize exosomes independent of phagocytic receptors 65 
3.14 Exosomes do not show tethering on the plasma membrane of microglia at $4^{\circ} \mathrm{C} \ldots \ldots \ldots \ldots$. . . . . . . . . . . . . 66

3.15 Uptake of exosomes is reduced in the presence of phosphatidylserine containing liposomes . . . . . . . . . . . . . . . . 67

3.16 MFGE8 is expressed by astrocytes in the murine brain . . . . . . . 69

3.17 Recombinant MFGE8 facilitates exosome internalization into microglia . . . . . . . . . . . . . . . . . 70

3.18 Exosomes and dextran are partially co-internalized into microglia . 71

3.19 Microglia internalize FITC-labeled myelin . . . . . . . . . . . . . . . 72

3.20 Internalized FITC-myelin is found in the late endosomal/ lysosomal compartment of microglia . . . . . . . . . . . . . . . . . 73

3.21 Exosomes and FITC-myelin partially co-localize after internalization by microglia . . . . . . . . . . . . . . . . . . . . . . 74

3.22 Exosomes do not lead to pro-inflammatory nor anti-inflammatory cytokine release of microglia . . . . . . . . . . . . . . . 76

3.23 Exosomes do not alter IFN $\gamma$ induced MHCII expression of microglia 77

3.24 Exosomal proteins are not presented via MHCII . . . . . . . . . . . 79

3.25 Inflammatory stimuli downregulate macropinocytosis of exosomes in microglia . . . . . . . . . . . . . . . . . . . . . 81

3.26 Microglia expressing MHCII show decreased exosome internalization 82

3.27 IFN $\gamma$ stimulated microglia show differential expression of B7-1 and $\mathrm{B} 7-2 \ldots \ldots \ldots \ldots \ldots \ldots$

3.28 Microglial subpopulation(s) show IFN $\gamma$ inducible MHCII expression in vitro . . . . . . . . . . . . . . . . . . . . . . . . . . . . . . . 85

3.29 Stimulation of MHCII expression in vivo by injection of IFN $\gamma$. . . 86 


\section{List of Tables}

1.1 List of cytokines and chemokines released by microglia [1] . . . . . 19

$2.1 \quad$ Antibodies . . . . . . . . . . . . . . . 27

2.2 Inhibitors, sugars and peptides . . . . . . . . . . . . 30

2.3 Commercial kits . . . . . . . . . . . . . . . . . . . . . . . 31

2.4 List of software . . . . . . . . . . . . . . . . 31

2.5 Directly-coupled antibodies (FACS) . . . . . . . . . . . 43 


\title{
List of Symbols and Abbreviations
}

\author{
aa Amino acid \\ AG Acetylglucosamine \\ ap Anterior-posterior axis \\ APS Ammoniumperoxodisulfat \\ BBB Blood brain barrier \\ BSA Bovine serum albumin \\ BCA Bicinchoninic acid \\ BME Basal medium eagle \\ APC Antigen presenting cell \\ APS Ammonium persulfate \\ CNPase 2',3'-cyclic-nucleotide 3'-phosphodiesterase \\ CNS Central nervous system \\ d $\quad \operatorname{Day}(\mathrm{s})$ \\ DC Dendritic cell \\ DMEM Dulbecco's modified eagle medium \\ DNA Deoxyribonucleic acid \\ dv Dorsoventral axis \\ EAE Experimental autoimmune encephalomyelitis \\ EBV Epstein-Barr-Virus \\ EDTA Ethylenediaminetetraacetic acid \\ EGF Epidermal growth factor \\ eGFP Enhanced green fluorescent protein \\ EGFR Epidermal growth factor receptor \\ ELISA Enzyme-linked immunosorbent assay
}


ER Endoplasmic reticulum

ESCRT Endosomal sorting complex required for transport

EtBr Ethidium bromide

FACS Fluorescence activated cell sorting

FcR Fc receptor

FCS Fetal calf serum

FDC Follicular dendritic cell

FITC Fluoresceinisothiocyanat

FU Fucoidan

GalC Galactosylceramide

GEF Guanosine exchange factor

GFP Green fluorescent protein

GM130 Golgi matrix protein, $130 \mathrm{kDa}$

GTPase Guanine triphosphatases

GTP Guanosine triphosphate

HBSS Hank's buffered salt solution

HEPES 4-(2-hydroxyethyl)-1-piperazineethanesulfonic acid

h $\quad \operatorname{Hour}(\mathrm{s})$

HRP Horse radish peroxidase

Iba1 Ionized calcium binding adaptor molecule 1

ICAM1 Inter cellular adhesion molecule 1

IFN $\gamma \quad$ Interferon gamma

IL Interleukin

ILV Intraluminal vesicle

i.p. Intra-peritoneal

IP-10 Interferon gamma-induced protein $10 \mathrm{kDa}$

i.v. Intra-venous

$\mathrm{kDa} \quad$ Kilodalton

Lamp1 Lysosomal-associated membrane protein 1

LDL Low density lipoprotein

LPS Lipopolysaccharide

LRPAP1 LDL-receptor-related protein associated protein1 


\begin{tabular}{|c|c|}
\hline MA & Mannosamine \\
\hline MAC1 & Macrophage antigen 1 \\
\hline MAG & Myelin-associated glycoprotein \\
\hline MBP & Myelin basic protein \\
\hline MCP1 & Monocyte chemoattractant protein 1 \\
\hline MCP2 & Monocyte chemoattractant protein 2 \\
\hline MCSF & Macrophage colony stimulating factor \\
\hline MFGE8 & Milk fat globule-EGF factor 8 protein \\
\hline $\mathrm{ml}$ & Medio-lateral axis \\
\hline $\mathrm{MOG}$ & Myelin oligodendrocyte glycoprotein \\
\hline $\min$ & Minute(s) \\
\hline $\mathrm{MIP} 1 \alpha$ & Macrophage inflammatory protein 1 alpha \\
\hline $\operatorname{MIP} 1 \beta$ & Macrophage inflammatory protein 1 beta \\
\hline $\mathrm{MHC}$ & Major histocompatibility complex \\
\hline MS & Multiple sclerosis \\
\hline MVB & Multivesicular body \\
\hline $\mathrm{P} 0$ & Postnatal day 0 \\
\hline PAGE & Polyacrylamide gel electrophoresis \\
\hline PAMP & Pathogen associated molecular pattern(s) \\
\hline PBS & Phosphate buffered saline \\
\hline $\mathrm{PC}$ & Phosphatidylcholine \\
\hline PCR & Polymerase chain reaction \\
\hline PFA & Paraformaldehyde \\
\hline pi & Post-injection \\
\hline PI & Phosphatidylinositol \\
\hline PLL & Poly-L-lysine \\
\hline PLP & Proteolipid protein \\
\hline pOLs & Primary oligodendrocytes \\
\hline PS & Phosphatidylserine \\
\hline Rab & Ras like protein in brain \\
\hline RANTES & Regulated upon activation, normal T-cell expressed and secreted \\
\hline RNA & Ribonucleic acid \\
\hline
\end{tabular}


ROS Reactive oxigen species

RT Room Temperature

SD Standard deviation

SDS Sodium dodecyl sulphate

SEM Standard error of the mean

SNARE Soluble NSF attachment protein receptor

$\mathrm{S}$

Second(s)

TAMRA Carboxytetramethylrhodamine

TAP Transporter associated with antigen processing

TCR T cell receptor

TEMED N'N'N'N'-tetramethylethylene diamine

TLR Toll-like receptor

TNF $\alpha \quad$ Tumor necrosis factor alpha

VAMP Vesicle-associated membrane protein

WGA Wheat germ agglutinin 


\section{Acknowledgments}

I want to thank Prof. Mikael Simons for giving me the opportunity to join his lab and for the supervision of my doctoral project. Without his scientific guidance and technical advisory this study would not have been possible. I also want to acknowledge my thesis committee members Prof. Uwe-Karsten Hanisch and Prof. Jürgen Wienands for their interest in my projects and inspiring discussions. To Dirk Fitzner, I express my gratitude for starting the exosome project and his contributions to its success.

I sincerely thank our collaborators: Denise van Rossum for FACS measurements and fruitful discussions on microglia, Tommy Regen for ELISA measurements, Gurumoorthy Krishnamoorthy for the T cell assays and Payam Dibaj for the 2-photon experiments. Special thanks go to Mostafa Bakhti for his last minute primary exosomes. I also want to acknowledge the Molecular Medicine PhD program for their administrative support during my doctoral thesis.

I am grateful to all members of AG Simons for their support and the inspiring atmosphere in the lab: Larisa Yurlova, Katrin Strauss, Sebastian Schmitt, Chieh Hsu, Angelika Kippert, Shweta Aggarwal, Natalia Manrique, Aniket Ghosh, Mostafa Bakhti, Johannes Zimmermann, Holger Budde, Christina Reetz, Olena Steshenko, Nicolas Snaidero, Hannes Treiber, Anja Schneider and Kirstin Fladung. I enjoyed the time I spent with you very much! Special thanks to Giselheid Schulz for primary cells and the creative application of "motivase".

I am indebted to my friends for their support. I especially want to thank Katrin Strauss, Larisa Yurlova and Sebastian Schmitt for welcoming me in Göttingen. Thank you for your company and joy during homeostasis and for good advice in pro-inflammatory times. I am grateful to Theresa Waldmann for her unshaken be- 
lief in the goodness of people and Tina Scheffler and Janis Schuller for keeping me in touch with the world outside of science. I thank my friends from my years of study, especially Lara Danielsen and Maike Laussmann, and my childhood friends sill holding the fort in Bremen.

Special thanks go to my entire family. I am particularly grateful to my parents Ursula and Manfred for their love and unlimited support, to my sister Kerstin for her reasoning powers in rough times, to my brother Henryk for taking care of our family and to my sister Wiebke for reminding me of the important things in life. 


\section{Abstract}

Oligodendrocytes are the myelin forming cells of the central nervous system. These cells synthesize vast amounts of membrane during the active phase of myelination. In addition, membrane components are released into the extracellular space as small vesicles, exosomes, with a size of 50 to $100 \mathrm{~nm}$. In this study we address the fate and function of oligodendroglial exosomes in the extracellular space. We find that exosomes purified from oligodendroglial precursor cell line and primary oligodendrocyte cultures were efficiently and selectively internalized by microglia without inducing a pro-inflammatory response. The internalization occurred by macropinocytosis that was partially dependent on exosomal lipid phosphatidylserine and autonomous of typical phagocytic receptors. Interestingly, we identified two subpopulations of microglia after stimulation with inflammatory cytokines. One subpopulation of microglia displayed antigen-presenting capacity due to major histocompatibility complex (MHC) class II expression, whereas the other microglial pool remained MHCII negative. However, exosome internalization occurred preferentially by microglia without antigen-presenting capacity. Based on these results, we propose that oligodendrocytes secrete exosomes into the extracellular space to employ microglia for the degradation of membrane components during myelination and membrane turnover. Moreover, the functional specialization of microglia supports the notion of heterogeneity in the microglial population. Yet, it remains to be elucidated whether the functional specialization of microglia is intrinsic or mediated by the microglial microenvironment. 


\section{Chapter 1}

\section{Introduction}

\subsection{Exosomes}

Eukaryotic cells use vesicles both for the intracellular transport and for secretion of cargo into the extracellular milieu. Besides the direct shedding of vesicles from the plasma membrane, vesicles can also arise from multivesicular endosomes by inward budding into their lumen and the subsequent fusion with the plasma membrane. After the release of internal vesicles, they are typically referred to as exosomes.

Exosomes were first discovered by Pan and Johnstone during reticulocyte differentiation $[2,3]$. Their group described vesicles present in large multivesicular endosomes containing transferrin receptors, which were previously internalized from the plasma membrane. The large endosomal structures fused with the plasma membrane and released the internal vesicles into the extracellular space. To date, various other studies have revealed the existence of exosomes in multiple cell types including oligodendrocytes [4-6], B lymphocytes [7], dendritic cells [8], platelets [9], epithelial cells [10] and neurons [11].

Exosomes have a size of 50 to $100 \mathrm{~nm}$ and a characteristic "saucer-shape", as visualized by electron microscopy [12], which is consistent with the size and morphology of internal vesicles in multivesicular endosomes [7]. Exosomes can easily be purified in vitro by sequential centrifugation of cell culture medium. This includes several centrifugation steps with increasing forces to eliminate cell debris, followed by ultracentrifugation at $100.000 \times \mathrm{g}$ for their pelletation [13]. In addition, exosomes exert a specific flotation pattern on sucrose gradients $(1.13 \mathrm{~g} / \mathrm{mL}$ to $1.19 \mathrm{~g} / \mathrm{mL})$, allowing 
their preparative differentiation from small sized vesicles of other origin [13]. The abundance of specific marker proteins represents an additional purity control (see Section 1.1.2).

The following subsections will elaborate on the formation and the composition of exosomes. Furthermore, their release and their functions in vivo will be addressed.

\subsubsection{Formation of exosomes}

Exosomes are thought to arise from the multivesicular endosomal compartments of the cell. In these multivesicular bodies (MVBs) membrane components are usually further transported to lysosomes for degradation of cargo (see Figure 1.1.1). Endosomes are classified according to their morphology, their distinct protein and lipid composition and their cargo as early, late or recycling endosomes $[14,15]$. In the endocytic pathway, internalized proteins and lipids are first incorporated into early endosomes $[16,17]$. During the maturation of the early to the late endosome, proteins and lipids are sorted into vesicles, which bud into the lumen of the endosome. These intraluminal vesicles (ILVs) accumulate in the endosomal compartment, forming a distinct late-endosomal compartment, the multivesicular body. Based on their biochemical properties, MVBs can either traffic to lysosomes for degradation of contents or fuse with the plasma membrane to release the intraluminal vesicles into the extracellular milieu, which are than called exosomes [13]. The docking/fusion process of the MVBs is likely to be mediated by SNARE proteins and synaptotagmin family members, since e.g. VAMP7, syntaxin7 and synaptotagmin7 are known to be implicated in the fusion of conventional lysosomes with the plasma membrane [18].

One of the mechanisms that underly the sorting of proteins into MVBs includes mono- and oligo-ubiquitination of the target proteins [19, 20]. The ubiquitinated proteins are recognized by the endosomal-sorting-complex-required-fortransport machinery (ESCRT), which promotes their inclusion into the ILVs [21]. The ESCRT-0, ESCRT-1 and ESCRT-2 complexes recognize and sort out the ubiquitinated proteins, while ESCRT-3 complex appears to be responsible for inward 


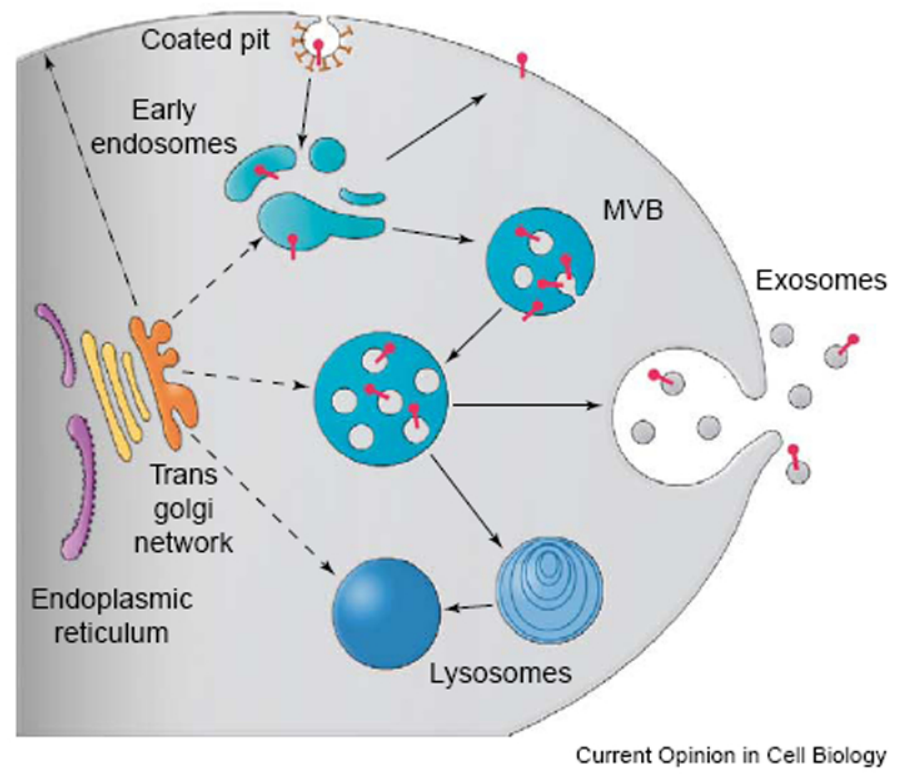

Figure 1.1: Multivesicular bodies fuse with the plasma membrane to release exosomes.

In early endosomes, membrane proteins (red) are either recycled to the plasma membrane or sequestered into internal vesicles. The budding of vesicles from the limiting membrane of endosomes leads to formation of multivesicular bodies (MVBs) containing intraluminal vesicles (ILVs). In the degradation pathway, MVBs fuse with the lysosomal system for degradation of proteins and lipids. In the alternative secretory pathway, MVBs fuse with the plasma membrane and release ILVs into the extracellular milieu, which are then called exosomes. Exosomes display the same orientation as the plasma membrane. The figure is adapted from Fevrier and Raposo (2004). Reprint by permission from Elsevier Limited: Current Opinion in Cell Biology, copyright (2004).

membrane budding $[22,23]$. However, also non-ubiquitinated proteins are sorted into intraluminal vesicles $[24,25]$.

\subsubsection{Lipid and protein composition of exosomes}

Lipid analysis of exosomes has been performed only on a small set of cell types including dendritic cells [40], mast cells [40], reticulocytes [41], B cells [35] and oligodendrocytes [5]. Nevertheless, it was shown that the lipid composition of exosomes 


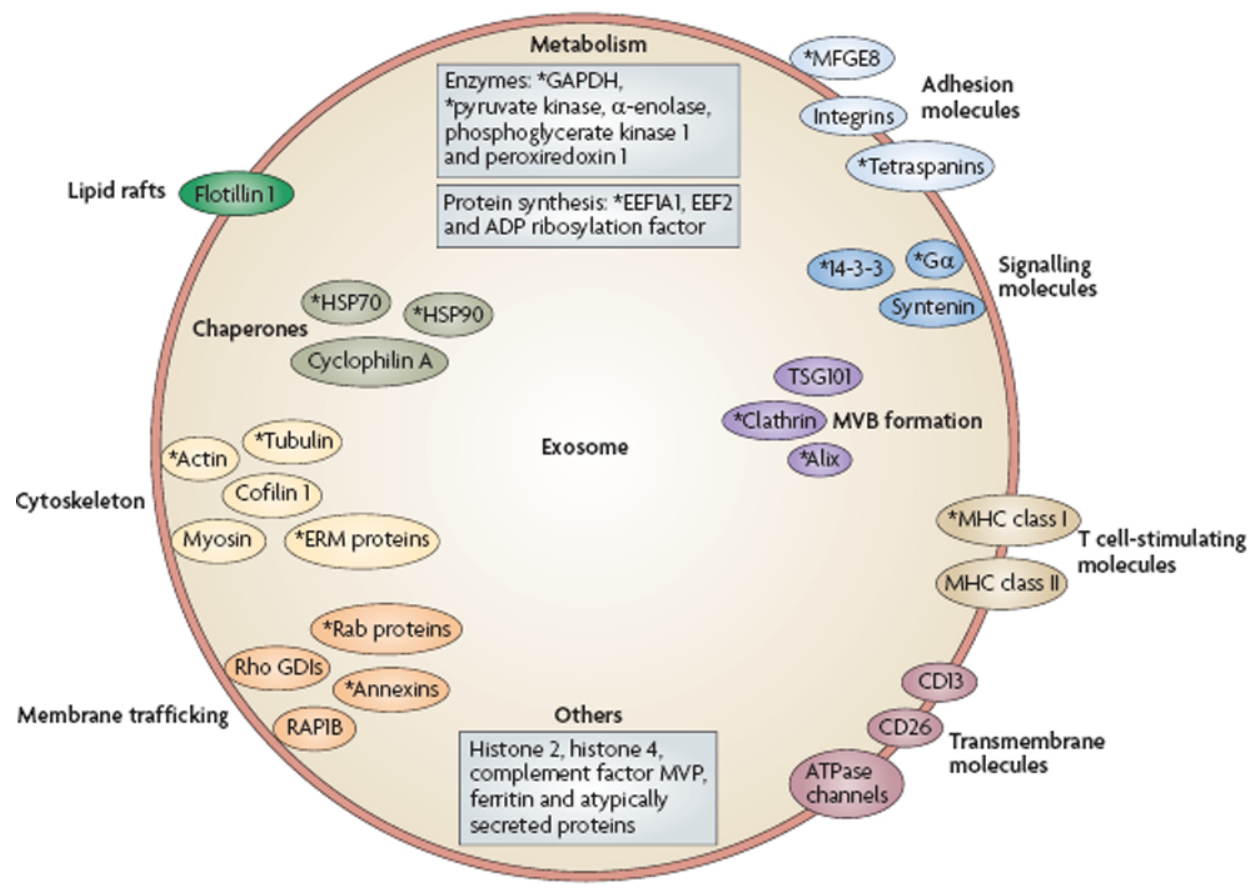

\section{Figure 1.2: Representative protein composition of exosomes.}

Protein composition of a typical exosome containing data retrieved from 15 proteomic analysis on exosomes [12, 26-39]. Proteins found in at least $30 \%$ of analyzed exosomes are indicated, proteins present in at least $50 \%$ are additionally marked by an asterix. The figure is adapted from Thery et al. (2009). Reprint by permission from Nature Publishing Group: Nature Reviews Immunology, copyright (2009).

reflects the lipid composition of their parenting cell type. In addition, exosomes are enriched in raft-lipids like cholesterol, sphingolipids, ceramide and glycophospholipids $[5,35,42]$.

Exosomes typically do not contain endoplasmatic reticulum, mitochondria or nuclear proteins, which is consistent with their endosomal origin [43]. The actual protein content of exosomes varies according to their cell type of origin, nonetheless they contain various common protein components $[33,44]$. In proteomic studies conducted on exosomes of 19 different cell types, a conserved set of proteins was identified [45]. Among them were cytosolic proteins as well as transmembrane proteins. Most abundant proteins are Alix and Tsg101, as part of the MVB biogenesis 
machinery, identified in $68 \%$ and $37 \%$ of the studies, respectively. Similarily, HSP70 was identified in $89 \%$ of the proteomic studies [46] (see Figure 1.2). Another class of cytosolic proteins commonly found in exosomes are Rab proteins, which play a role in the docking of exosomes and membrane fusion events . In total 40 different Rab proteins were found in exosomes [33]. Furthermore, tetraspanins including CD9, CD63, CD81 and CD82 are typical exosomal proteins [47]. As they can also form a protein network, tetraspanins could be involved in the generation of ILVs [24]. Even the recruitment of other membrane proteins from the limiting membrane of endosomes into ILVs could be mediated by early incorporation into tetraspanincontaining detergent-resistant membrane domains [35]. Moreover, exosomes carry lipid raft proteins like Flotillin-1 [43] and cell adhesion proteins like intercellular adhesion molecule-1 (ICAM-1), CD146, CD9, MFGE8 and CD18 [33, 44].

Besides the protein set found in almost all released exosome types, exosomes also contain proteins, which are highly specific for their parenting cell. Exosome derived from antigen presenting cells carry MHCII and CD86 molecules necessary for antigen presentation and $\mathrm{T}$ cell stimulation [47]. $\mathrm{T}$ cell receptors are also specifically enriched in T cell-derived exosomes [48].

Oligodendrocytes, as the myelinating cells of the CNS, secrete exosomes enriched in myelin proteins, like the proteolipid protein (PLP) [4-6] (see Section 1.2.1).

\subsubsection{Release of exosomes}

Depending on the parenting cell type the secretion of exosomes can be spontaneous or induced. While reticulocytes [2], T cells [48, 49] and resting B cells [50] secrete detectable levels of exosomes only after stimulation of a cell surface receptor, dendritic cells [8] and macrophages [51] show a developmentally regulated or a constitutive exosome secretion, respectively.

The molecular machinery regulating the fusion of MVBs with the plasma membrane is still under investigation. It is also not known whether there are different kinds of multivesicular bodies, destined for different fates or whether MVBs contain intermixed classes of intraluminal vesicles, which later on segregate.

The release of exosomes could use similar mechanisms as applied during the fusion 
of secretory lysosomes with the plasma membrane [52]. As shown for lysosomal exocytosis, the treatment of oligodendrocytes with the calcium-ionophore ionomycin increased the release of exosomes [4]. This has also been shown for the exosome release of other cell types including epithelial cells and neurons [31, 53]. Therefore exosome release might be regulated via intracellular calcium levels [4].

Calcium-induced lysosomal exocytosis requires Rab27a, which primes vesicles for fusion with the plasma membrane [54]. Both small GTPases Rab27a and Rab27b, are involved in docking of MVBs with the plasma membrane. The knock-down of Rab27a strongly increases MVB size, Rab27b silencing results in MVB redistribution towards the perinuclear region, while both lead to a decrease in exosome secretion. This suggests a role of both homologous proteins in the exosomal pathway [55]. In addition, Rab11-GTPase activity was implicated in the fusion of MVBs in response to $\mathrm{Ca}^{2+}$ [56]. Studies using overexpression of Rab11 and citron kinase, a RhoA effector, could show an upregulation of exosome release $[56,57]$. In oligodendrocytes, exosome release is dependent on the Rab GTPase-activating protein family, TBC1D10, and its target Rab35. Inhibition of Rab35 leads to the intracellular accumulation of vesicles and a decrease in exosome secretion [6].

Furthermore, the decrease of membrane cholesterol or the inhibition of cholesterol biosynthesis results in an increase of exosome release [58-60]. This might be due to the cholesterol-dependent regulation of membrane-associated Rab7 influencing the rate of MVB transport to the plasma membrane [61]. Cellular stress, such as DNA damage or heat shock, has been shown to stimulate exosome secretion via tumor supressor p53 [59, 62].

The developmental regulation of exosome release was described in dendritic cells. Several groups could show that LPS-stimulated, mature DCs reduce exosome release by up to $75 \%$ in compairison to immature DCs $[47,63,64]$.

\subsubsection{Function of exosomes}

\section{Pathway of protein and lipid disposal}

Different functions of exosomes have been hypothesized in the course of their discovery in various cell types. The first function was described by Pan and colleagues, who 
showed the implication of exosomes in the elimination of proteins such as transferrin receptor or integrins during the differentiation of reticulocytes [2, 25]. Already in 1984, they revealed that reticulocytes lose their surface transferrin receptors during maturation into erythrocytes in vitro. This removal is executed via receptor-enriched multivesicular domains, which bud into the extracellular milieu [65]. But exosomes are not only an alternative pathway for the degradation of proteins. A study on oligodendroglial cells illustrated that exosomes contribute to the cellular cholesterol homeostasis. Especially in lysosomal storage diseases, exosomes might constitute a pathway which partially bypasses the toxic accumulation of lipids in the endosomal system [66]. Therefore, the release of proteins and lipids via the exosomal route might represent an alternative pathway of disposal for the parenting cells. However, this fact raises even more questions regarding their fate in the extracellular milieu and putative signaling functions of exosomes.

\section{Intercellular communication}

As exosomes contain proteins and lipids of their parenting cells, it is highly likely that they are involved in signaling events. Beyond the clearing function of exosomes, the presence of adhesion proteins on exosomes may points to other roles of exosomes. Phenotypic and functional analysis of exosomes from immune cells support the idea that exosomes establish a novel mode of cellular communication. This includes both extracellular docking of exosomes to receptors of the target cells and the exchange of exosome cargo between exosome-producing and the respective target cell.

The first studies showing an alternative function of exosomes were performed by Raposo and colleagues, who demonstrated that exosomes released by EBV-transformed B cells contained functional MHCII molecules, which were capable to stimulate human $\mathrm{CD}^{+} \mathrm{T}$ cells in vitro [7]. Thus, exosomes might carry both peptide-MHC complexes and antigenic material itself. Due to the presentation of biologically relevant antigens exosomes have been used in vaccination studies [67]. Zitvogel and colleagues showed in 1998, that exosomes produced by mouse DCs pulsed with tumor peptides induce the rejection of established tumors in vivo in a $\mathrm{T}$ cell mediated fashion [8]. Moreover, exosomes are even capable of transferring antigens from tumor cells to dendritic cells [68], which then could serve as source of antigen for 
cross-presentation by DCs. This became obvious in experiments, where tumor cellderived exosomes internalized by DCs induced tumor rejection in vivo [68].

Exosomes have also been implicated in immune suppression, for example in a delay of allograft rejection in rats [69]. This delay is likely to be due to a decrease in $\mathrm{CD} 4^{+}$ $\mathrm{T}$ cells in the exosome treated recipient. Another application inducing tolerance are exosomes produced by intestinal epithelial cells. These "Tolerosomes" are described to induce tolerance to oral antigens $[26,70,71]$.

Finally, exosomes have been implicated in the the transfer of non-protein cargo from parenting to target cells. Valadi and colleagues described exosomes derived from mast cell lines, which were enriched in messenger RNA and microRNA. These exosomes were able to shuttle RNA to neighboring cells where they were transcribed and biologically active [72]. Exosomes released by astrocytes and gliobastoma cells even carry mtDNA [73].

\section{Exosomes in vivo and their application in diagnostics}

Exosomes can not only be purified from cell cultures, but are also found in multiple body fluids including urine [38], amniotic fluid [59], bronchoalveolar lavage fluid [74] and plasma [75]. The physiological significance of exosomes is still not fully understood, since the in vivo description of exosome release is a challenging task. Nevertheless, interesting findings in tonsil germinal centers described MHCIIbearing exosomes attached to follicular DCs (FDCs), which are most likely derived from B cells [76]. This observation is of particular interest, because FDCs contain MHCII molecules but do not synthesize them [77]. These findings suggests a potential transfer of MHCII from exosomes to FDCs in vivo.

The presence of exosomes in body fluids lead to their application in diagnostic purposes. In studies comparing the exosome abundance in the plasma of cancer patients, the amount of exosomes was increased four times in comparison to healthy individuals $[75,78,79]$. Besides the increase of exosome levels in patients with advanced cancer, these findings also revealed that exosomes can travel far from their secreting cells [78]. In a recent study, prostate cancer biomarkers were detected in exosomes isolated from the urine of prostate cancer patients [80]. In addition, 
proteomic analysis of urine derived exosomes described 1132 proteins out of which 177 proteins are linked to disease-related genes [81]. 


\subsection{Glia cells in the central nervous system}

The mammalian central nervous system (CNS) consists of two major cell types, glia cells and neurons. The glial population can be classified into macroglia, namely oligodendrocytes and astrocytes, and microglia. All types of glia have a specialized function supporting the functional integrity and the long-term survival of the neuronal network [82].

Oligodendrocytes are the myelin forming cells in the CNS. After expanding their plasma membrane into multiple cellular processes, oligodendrocytes subsequently wrap their membrane around axons to form multilamellar sheaths. These sheaths are composed of a unique, lipid-rich membrane containing a limited set of myelin proteins. Each oligodendrocyte extends numerous cell processes that terminate in up to 50 myelin internodes on multiple axons. These membrane sheaths have insulating capacities facilitating the rapid saltatory signal conduction [82, 83]. Moreover, oligodendrocytes exert a neurotrophic function [84] (see Section 1.2.1). Perturbations of the myelin sheath results in several severe brains diseases, including multiple sclerosis (see Section 1.2.5).

The second type of macroglia, astrocytes, provide scaffolding functions for migrating neurons during fetal brain development. In the adult brain, astrocytes are found to enclose synaptic junctions and nodes of Ranvier and to secrete trophic factors. Furthermore, astrocytes take an active part in the supply of ions and metabolites for neurons and the regulation of the extracellular environment [85]. Similar to neurons, astrocytes are functionally coupled over large areas of the brain. Besides cell-contact-mediated communication via gap-junctions, astrocytes also communicate by extracellular signaling molecules [84].

Microglia represent the third type of glial cells in the CNS. They are the principal immune effector cell in the brain and are competent phagocytes [86]. Microglia monitor their microenvironment and notice changes in the brain homeostasis. In response to perturbations, microglial cells alter their activation status by up-regulating the expression of ion channels, cell surface molecules for adhesion, complement binding and antigen presentation. Furthermore, an increase in motility and phagocytic activity is commonly associated with transition, as well as the synthesis of a set 
of soluble factors, including cytokines and chemokines with potent immuno- and neuroregulatory effects [87, 88] (see Section 1.2.2).

The following subsections will elaborate on different aspects of development, function and interrelation of oligodendrocytes and microglia.

\subsubsection{Oligodendrocytes}

Oligodendrocytes in the CNS generate large extensions of their plasma membrane, which are wrapped around multiple axonal segments in a multilayered fashion. The myelin membrane thus forms a tightly compacted and insulating entity with a characteristic composition [82].

\section{Composition of the myelin membrane}

The myelin membrane is highly enriched in lipids (approx. $70 \%$ of its dry weight), in particular in cholesterol and the glycosphingolipids galactosylceramide (GalC) and sulfatide [89]. Additionally, several hundreds of different proteins have been detected by gel-based proteome analysis [90]. The major myelin proteins are the proteolipid protein (PLP) and its alternative splice form DM20, representing 50\% of total myelin proteins. The second most abundant myelin protein is the myelin basic protein (MBP) constituting 20 to $30 \%$ of the CNS myelin protein content. Further myelin proteins are the 2'-3'-cyclic nucleotide 3'-phosphohydrolase (CNP) [91] and the myelin-associated glycoprotein (MAG), which has two isoforms of 64 and $69 \mathrm{kDa}$. Another minor myelin component is the myelin/oligodendrocyte glycoprotein (MOG) [92]. The myelin sheath is characterized by both compact and non-compact membrane regions, which also differ in their protein composition [89]. Typical compact-myelin proteins are PLP and MBP, the latter facing the cytosolic membrane surface. MBP is instrumental in the process of myelination, as it mediates the compaction of the myelin membrane [89]. Both are found in the compacted internodal regions. $\mathrm{CNP}$ and MAG are localized in the non-compacted areas of myelin [93]. 


\section{Origin of oligodendrocytes}

Oligodendrocytes originate as neuroectodermal cells of the subventricular zones and mature, after migration, into postmitotic myelin-producing cells [94]. The earliest precursor Pre-GD3 differentiates into the proliferative and migratory bipolar O2A precursors which can differnetiate into both astrocytes and oligodendrocytes [95]. Lineage progression proceeds via postmigratory pro-oligodendroblasts [96] to immature oligodendrocyte stage, identified by synthesis of GalC, sulphatide and CNP. Finally, mature oligodendrocytes develop that express terminal differentiation markers MBP, PLP and MOG [83].

\section{Oligodendroglial exosomes}

Oligodendrocytes secrete exosomes enriched in myelin proteins PLP, CNP, MBP and MOG [4-6]. In oligodendrocytes, the lipid ceramide has been implicated in the budding of exosome-associated membrane domains into the lumen of endosomes [5]. It is thought that the formation of ILVs is fascilitated by membrane invaginations due to the cone shaped structure of ceramide $[5,97]$. Whereas ceramide was shown to be necessary for the sorting of cargo into the exosomal pathway, membrane destined for lysosomal degradation is sorted by the ESCRT machinery [5]. Furthermore, the treatment of oligodendrocytes with the calcium-ionophore ionomycin increases the release of exosomes [4].

\subsubsection{Microglia}

Microglia constitute 10 to $20 \%$ of the total population of glial cells in the adult mammalian CNS [98]. They are suggested to originate from the hematopoietic cell population and invade the CNS during development [99]. Postnatally, they are found in all regions of the CNS in a non-overlapping territorial fashion [100]. Microglia ptake part in tissue repair after injury, similar to that of resident macrophages in peripheral organs. Micoglial cells express most common macrophage markers including the calcium-binding adaptor molecule 1 (Iba1), Fc receptor (FcR), integrin- $\alpha \mathrm{M}$ (MAC-1/CD11b) and F4/80 [101, 102]. Based on their spatial localization within the CNS, microglia are divided into two groups, parenchymal microglia and perivas- 
cular microglia. They have a highly branched morphology, with each cell soma decorated by long processes with fine termini [103] (see Figure 1.3). Under physiological CNS conditions microglia continually survey their microenvironment through motile processes and membrane protrusions. The dynamic motility of resting microglia is partly directed towards synapses, to which they send out their processes with a frequency dependent on neuronal activity. Recent findings propose that microglia monitor and respond to the functional status of synapses [104]. While the parenchymal microglia are a stable self-renewing population $[105,106]$, the perivascular microglia population is rapidly replenished by bone marrow stem cells [107].

Under pathological conditions like infectious diseases or neurodegenerative processes, microglia change their "surveying status" [108]. They migrate to and within the lesion site while releasing a wide range of soluble factors. This includes cytotoxins, such as free oxigen intermediates or NO, neurotrophins and immunemodulatory factors [108-110]. Disruption of the blood-brain-barrier results in focal activation and active shielding of the injured site $[111,112]$. Studies using transcranial twophoton microscopy identified highly dynamic and rapid responses of microglia towards a site of traumatic injury, which were attracted by local release of ATP [103]. Importantly, peripheral macrophages and immune cells of the adaptive system are infiltrating the CNS during neuroinflammation to support the innate immune system. As professional phagocytes microglia can engulf invading microorganisms, remove potentially deleterious debris, promote ensuing tissue repair by secreting wound-healing factors and thus facilitate the return to tissue homeostasis.

\subsubsection{Innate immunity mediated by microglia}

The brain has a very effective barrier system based on tight junctions of the vasculature, the choroid plexus and the meningeal interfaces that prohibits free access of serum components and blood cells to the brain tissue. As long as the blood brain barrier $(\mathrm{BBB})$ is intact, the CNS remains largely separated from the peripheral immune system [110]. In the intact brain the direct interaction of microglia with 


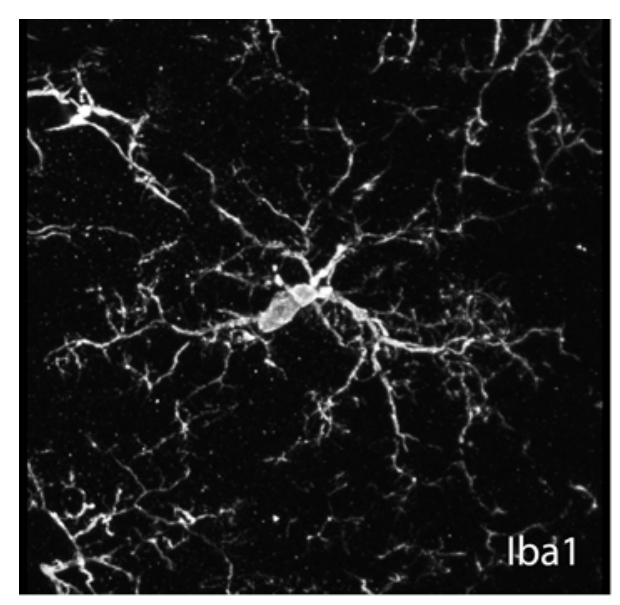

Figure 1.3: Morphology of a microglial cell in the murine hippocampus.

3D-reconstruction of a microglial cell in the adult murine hippocampus with highly branched processes. Mareike Schnaars, unpublished data.

pathogens and other immune cells is however limited. During pathological processes the blood-brain barrier becomes leaky and immune cells can infiltrate the CNS to mount the adaptive immune response. Whereas naive $\mathrm{T}$ cells are largely excluded from the CNS, activated T cells can migrate across the BBB, regardless of antigen specificity [113]. However, the resident microglia immediately support the initiation of the innate immune response.

Pathogens invading the CNS are recognized through receptors activated by pathogen associated molecular patterns (PAMPs). The largest class of pattern recognition receptors are the Toll-like receptors (TLRs), which are also expressed by microglia [114]. A large family of TLRs, consisting of 10 TLRs in human and 12 TLRs in mice has been characterized, which recognize patterns produced by bacteria, viruses, parasite and fungi [115]. In addition to ligands produced by infectious microorganisms, few proteins of the host are thought to be ligands for TLRs. Recognition of the endogenous ligands, such as HSP70, is likely to be a natural defense mechanism for the host to initiate an immune reaction in presence of damaged tissue [116]. Besides TLRs, microglia express nucleotide-binding oligomerization domain (NOD) proteins and non-TLR receptors, that are implicated in the recognition of PAMPs [117]. The binding of PAMPs to the respective microglia initiates the phagocytic 
removal of the pathogen (see Section 1.3). Moreover, the ligand-receptor interaction is triggering a pro-inflammatory phenotype of the cell and leads ultimately to the release of pro-inflammatory cytokines, as well as chemokines, proteases and redox proteins that help in tissue defense [117]. Another important facet of TLR ligation is the subsequent upregulation of co-stimulatory molecules, necessary to recruit and activate the cells of the peripheral immune system. This constitutes a way of bridging the innate and the adaptive immune responses [118].

\subsubsection{Antigen presentation by microglia}

Three populations of cells have the potential to act as antigen presenting cells (APCs) in the CNS: parenchymal microglia and perivascular macrophages, both resident APCs, and CNS-infiltrating inflammatory macrophages/dendritic cells [102], which originate in the bone marrow [119].

\section{Antigen-presenting cells}

Since whole proteins are not recognized by T cells, antigen-presenting cells must process the captured antigen by cleaving the protein into antigenic peptides. Among the most efficient APCs are dendritic cell (DC), which are found in all peripheral tissues and accumulate at the sites of of pathogen entry [120]. Immature DCs express a variety of phagocytic receptors, as well as Toll-like receptors and have a high phagocytic activity [121]. Early immature DCs in peripheral tissue concentrate their MHC class II molecules intracellularly [122]. After ingestion of pathogens, they undergo maturation and differentiate through an intermediate state, into late DCs, which exhibit almost all of MHCII on their plasma membrane [122]. As competent antigen presenting cells, DCs process antigens derived from pathogens, apoptotic cells or infected cells into peptides and load them onto MHC class I or class II molecules [120] (see below).

After capture of antigenic proteins derived from pathogens or cellular debris, tissue macrophages/ DCs migrate to the draining lymph nodes to present the antigen to $\mathrm{T}$ cells and initiate the primary immune response. While $\mathrm{CD} 4^{+} \mathrm{T}$ cells are activated 
by antigen presented on MHCII molecules, $\mathrm{CD} 8^{+} \mathrm{T}$ cells are stimulated by antigenloaded MHCI molecules [102]. Upon activation, T cells leave the lymph node and circulate trough the body searching for their target antigen. Within the target tissue, local APCs present the target antigen and retain the activated $\mathrm{T}$ cells at the site of damage or pathology. Many factors, including the affinity of the TCR for its antigen, the expression levels of MHCII, co-stimulatory molecules and chemokines define their future fate. This can lead to $\mathrm{T}$ cell proliferation or differentiation of effector functions [102]. Like other APCs, DC are specialized in linking the innate immune system with the adaptive immune response [120].

\section{Major histocompatibility complexes}

Presentation of protein-derived antigens to the immune effector cells is mainly accomplished by two protein complexes expressed by antigen-presenting cells. The major histocompatibility complex class I (MHCI), which is largely mediating the presentation of endogenous antigens and the major histocompatibility complex class II (MHCII), used for presentation of exogenous antigenic material [123-125].

MHCI complexes are comprised of a membrane-linked heavy chain, a soluble light chain and a short peptide bound to a groove within the heavy chain. All subunits are typically assembled in the ER through a elaborate assembly pathway. In the classical pathway, endogenous proteins from the cytosol are processed to peptides by the proteasome and transported into the ER lumen by the transporter associated with antigen-processing (TAP) [123]. The peptide loading complex supports the loading of peptide onto the MHCI complex and the peptide-occupied MHCI exits from the ER and transits to the cell surface where $\mathrm{T}$ cell activation takes place. In the non-classical pathway, exogenous antigens can be taken up by APCs trough endocytic mechanisms (see Section 1.3) and further processed for presentation via MHCI molecules. This process called "cross-presentation" is triggering a $\mathrm{CD}^{+}$ $\mathrm{T}$ cell response to exogenous antigens [123].

The second class of major histocompatibility complexes, MHC class II molecules, is comprised of heterodimers of two transmembrane proteins. After biosynthesis, both subunits assemble in the ER together with a short invariant peptide chain [124, 125]. 
Following the transport to the Golgi complex, most of the MHC class II molecules are transfered to the endocytic compartments of the cell $[126,127]$. After processing of exogenous antigens within the endocytic compartments, peptides are loaded on MHC class II molecules and further processed to 10 to 20 amino acid peptides. Finally, the peptide-loaded complex is translocated to the cell surface, where it is recognized by $\mathrm{CD} 4^{+} \mathrm{T}$ cells.

\section{Antigen presentation by microglia}

The microglia within the CNS parenchyma are long lived and have a low turnover rate as compared to perivascular macrophages [128]. In healthy adult humans, these parenchymal microglia are shown to be MHC class I and II negative and thus incapable of acting as antigen-presenting cells [110, 129]. However, the MHC class II expression is upregulated in human parenchymal microglia after damage or during immune reaction [130]. In vitro studies on unstimulated microglia showed, that these cells can not present antigens. Upon activation by interferon- $\gamma(\operatorname{IFN} \gamma)$ or by viral infection, microglia are stimulated and subsequently able to process and present viral antigens and exogenous myelin antigens to T cells [131]. Further studies revealed that after stimulation with $\operatorname{IFN} \gamma$ or tumor necrosis factor- $\alpha$ (TNF $\alpha$ ), the expression of T cell costimulatory receptors like ICAM-1, CD40, B7-2 and B7-1 is dramatically upregulated $[129,132]$. Hence, microglia are under certain circumstances capable to interact with T cells in an antigen-specific manner [102].

In addition to parenchymal microglia, macrophage populations localize to the perivascular space, the choroid plexus and the meninges. These microglia/ macrophage populations display a different phenotype compared to parenchymal microglia, probably due to their distinct microenvironment [133]. Perivascular macrophages constitutively express MHC class II $[134,135]$ and can present presentation, even without preceding stimulation.

Although in vitro studies suggest that microglia can present antigen, there is no evidence yet that microglia are capable of moving to the cervical lymph nodes like mature DCs. This step is crucial in the initiation of the primary immune response, since $\mathrm{T}$ cells need to be stimulated by antigen bearing APCs prior to their circulation to the site of pathology [102]. So far it could only be shown that the 
antigen-presenting microglia is awaiting the already primed $\mathrm{T}$ cell within the CNS. Despite the blood-brain-barrier, activated T cells easily infiltrate the CNS [105, 136], where microglia can retain and restimulate already primed T cells [137]. Depending on the microenvironment, this can lead to $\mathrm{T}$ cell proliferation, $\mathrm{T}$ cell anergy or even T cell apoptosis [102].

\section{Chemokines and microglia}

To fulfill their role in the innate immunity, microglia are equipped with appropriate sensors to monitor alterations in soluble and insoluble factors in their microenvironment [1]. The interaction of chemokines and their receptors is complex and reflects some redundancy in the system, as individual chemokines can activate several different chemokine receptors and conversely, individual chemokine receptors can often be activated by several different chemokines [138].

The production of chemokines by resident cells is crucial for the recruitment of $\mathrm{T}$ cells into the inflamed CNS. Among the cell types identified as sources of chemokines are microglia, astrocytes, neurons and endothelial cells [138]. A large set of different chemokines are upregulated during pathological states. Among these are fractalkine/CX3CL1, as well as the inflammatory chemokines MCP-1/CCL2, MCP2/CCL8, RANTES/CCL5, MIP1 $\alpha /$ CCL3, MIP1 $\beta$ /CCL4, IL-8/CXCL8 and IP-10/ CXCL10 [138].

The chemokine fraktalkine is widely expressed in the brain and localizes principally to neurons, as a membrane-bound ligand, whereas its receptor is found exclusively in microglia and astrocytes [138]. Interestingly, fractalkine induces proliferation of microglial cultures. The chemokines CCL2, CCL3, CCL4, CCL5 and CXCL10 have a role in the attraction of $\mathrm{T}$ cells and monocytes/ macrophages [119]. In contrast to its pro-inflammatory functions, the chemokine RANTES/CCL5 seems to exert additionally a vital role in the brain development by influencing the development of astrocytes. A proliferative activity on astrocytes has been found for MIP1 $\alpha$ and MIP1 $\beta$ as well [138]. 
Table 1.1: List of cytokines and chemokines released by microglia [1]

\begin{tabular}{llll}
\hline Cytokine/ chemokine & Abbreviation & Cytokine/ Chemokine & Abbreviation \\
\hline Growth regulated oncogene $\alpha$ & Gro $\alpha$ & gamma interferon inducible protein-10 & IP-10 \\
Interleukin-1 $\alpha /-1 \beta$ & IL-1 $\alpha /$ IL-1 $\beta$ & monocyte chemattractant protein-1 & MCP-1 \\
Interleukin-1 receptor antagonist & IL-1ra & macrophage colony stimulating factor & M-CSF \\
Interleukin-3 & IL-3 & macrophage derived chemokine & MDC \\
Interleukin-6 & IL-6 & macrophage inflammatory protein-1 $\alpha$ & MIP-1 $\alpha$ \\
Interleukin-8 & IL-8 & macrophage inflammatory protein-1 $\beta$ & MIP-1 $\beta$ \\
Interleukin-10 & IL-10 & macrophage inflammatory protein-2 & MIP-2 \\
Interleukin-12 & IL-12 & macrophage inflammatory protein-3 $\beta$ & MIP-3 $\beta$ \\
Interleukin-15 & IL-15 & regulated on activation, & RANTES \\
Interleukin-18 & IL-18 & normal T cell expressed and secreted & \\
Transforming growth factor $\beta$ & TGF $\beta$ & Tumor necrosis factor $\alpha$ & TNF $\alpha$ \\
\hline
\end{tabular}

\section{Cytokines and microglia}

Besides chemoattractive chemokines, further cytokines were found to play a role in the intercellular communication within the CNS. Under physiological conditions, cytokines are produced in low levels in the CNS, however after injury cytokines are transiently upregulated and secreted by many cell types including microglia [139]. In addition, viral envelopes, bacterial wall components and other infectious agents are efficient inducers of cytokine release [1]. Multiple cytokines and their receptors have been found to be present and functional in the CNS. Among them are TNF $\alpha$, interferons, IL-1, IL-2, IL-3, IL-4, IL-6, IL-10, IL-12, IL-15 and IL-18, $\mathrm{TGF} \beta, \mathrm{MCSF}, \mathrm{PDGF}, \mathrm{EGF}$ and neurotrophic factors [1]. IFN $\gamma$ and $\mathrm{TNF} \alpha$ are potent pro-inflammatory cytokines produced by key participants of the adaptive immune response (e.g. NK cells, CD4 ${ }^{+} \mathrm{T}$ cells) and known to shift the microglial phenotype towards pro-inflammatory state [140]. Interestingly, TNF $\alpha$ is also secreted by microglia, as well as pro-inflammatory cytokines IL-1 $\beta$ and IL-6. Another example are the colony-stimulating factors (CSFs), including IL-3, macrophage-CSF and granulocyte-macrophage-CSF. They have been implicated as key determinants of pro-inflammatory microglial activity [141-143]. To limit the course of an induced pro-inflammatory response, several negative feedback mechanisms are operating. The conversion of microglia from pro-inflammatory into an immun-modulatory phenotype is a critical component due to the switch from the production of pro- 
inflammatory to anti-inflammatory cytokines [139]. The interleukins IL-4, IL-10, IL-13 and TGF $\beta$ for example act on microglia and suppress many IFN $\gamma$ - induced functions including the expression of MHCII and costimulatory molecules CD40 and B7-2 as well as the production of pro-inflammatory TNF $\alpha$ and IL-1 $\beta$. All cytokines and chemokines released by microglia are summarized in Table 1.1 [1].

\subsubsection{Microglia and multiple sclerosis}

Multiple sclerosis (MS) is an inflammatory demyelinating autoimmune disease of the CNS [144]. Its onset is usually between 20 and 40 years of age [145] and the disease leads to substantial neurological disability in the majority of the patients [146]. There are two major forms of MS: the relapsing-remitting form, with 85 to $90 \%$ of all cases, and the primary progressive MS. Most patients with relapsing-remitting MS develop a secondary progressive MS later on. Both environmental and genetic factors have been implicated in the susceptibility to multiple sclerosis. For example, one or more susceptibility genes are located on chromosome 6p21 in the area of the major histocompatibilty complex, which is thought to account for 10 to $60 \%$ of the genetic risks of MS [147, 148].

Microglia contribute to the pathology of MS and its model experimental autoimmune encephalitis (EAE) through antigen presentation and secretion of pro-inflammatory cytokines [149]. Heppner at al. revealed that the paralysis of microglia inhibits the development and maintenance of inflammatory lesions. In their studies, they used transgenic mice that express the suicide gene HSVTK under the CD11b promoter. After application of ganciclovir, they observed ablation of macrophages, while parenchymal microglia were still vital but unable to release immun-modulatory molecules that either damage tissue directly or attract immune cells including autoreactive T cells in EAE [112].

Findings by Becher and colleagues described the importance of one microglial signaling molecule in the development of MOG induced EAE. In transgenic mice lacking microglial expression of IL-23, the clinical symptoms of MOG-EAE were drastically reduced. Although there was little impact on the degree of inflammation, the $\mathrm{T}$ cell 
cytokine profile shifted from a pro-inflammatory Th1 response to a protective Th2 response [150].

Besides the negative contributions of microglial cells in MS and EAE pathology, there is also evidence emphasizing a potential beneficial role of microglia in the course of MS. Next to secretion of neurotrophic factors [151], microglia also release anti-inflammatory cytokines (IL-10 and TGF $\beta$ ) [152]. Microglia are able to change the inflammatory state of the microenvironment and promote neurogenesis. Thus, microglial cells may perform both neuro-destructive and neuro-protective functions [153]. 


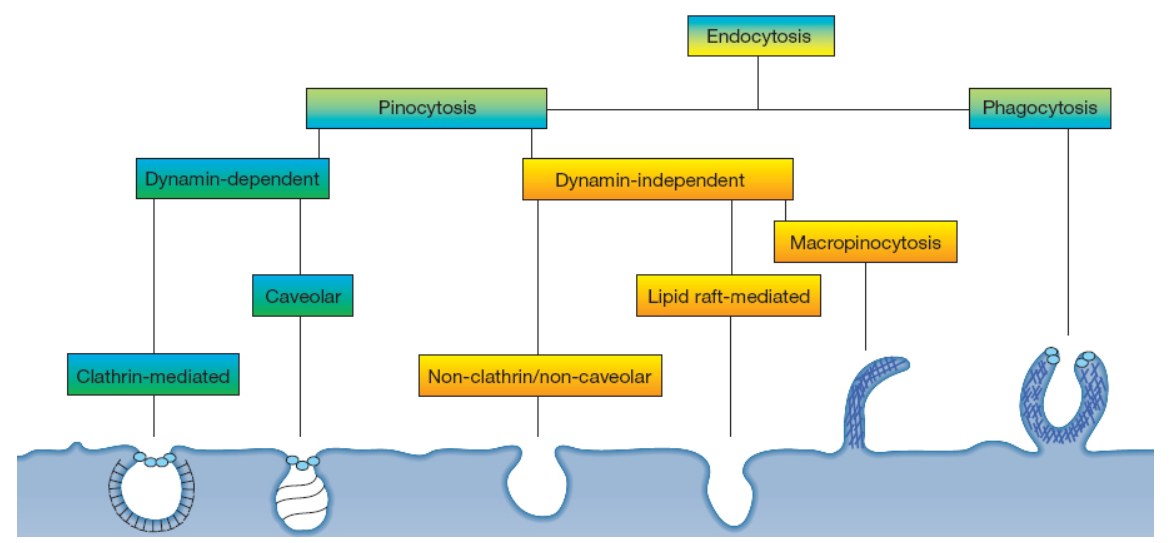

Figure 1.4: Endocytic pathways in mammalian cells.

Endocytic mechanisms are divided into two main classes depending on whether uptake involves mainly fluid and solutes (pinocytosis) or large particles (phagocytosis). Phagocytosis is restricted to phagocytic cells, while pinocytosis encompasses several distinct mechanisms that can be further divided by the implication of distinct proteins. Note that not the entire range of endocytic mechanisms and active proteins is depicted. The figure is adapted from Mercer and Helenius (2009). Reprint by permission from Nature Publishing Group: Nature Cell Biology, copyright (2009).

\subsection{Endocytic pathways in mammalian cells}

There are many distinct endocytic pathways that coexist in mammalian cells. A common feature of all pathways is the coordinated action of proteins capable of deforming the plasma membrane to form highly curved endocytic intermediates and proteins that can induce scission of these intermediates from the plasma membrane [154]. Nevertheless, the processes differ in the nature of the cargo, the cellular factors involved and the fate of the internalized material. Among the variety of strategies to internalize particles and fluids are phagocytosis and a group of pinocytotic mechanisms, including clathrin- or caveolin-mediated endocytosis and macropinocytosis (see Figure 1.4). The following subsections will elaborate on phagocytosis and macropinocytosis, both are endocytic mechanisms active in microglia. 


\subsubsection{Phagocytosis}

Phagocytosis by macrophages is critical for the uptake and degradation of infectious agents and participates in development, tissue remodeling, immune response and inflammation. Since phagocytosis of apoptotic cells is a constitutive physiological process, the mechanisms enabling marcrophages to recognize, bind, internalize and degrade apoptotic cells need to function without activating the pro-inflammatory responses of the macrophage. In order to phagocytose apoptotic cells, macrophages must encounter a ligand, which is not present on live cells. Ligands known so far to fit these criteria are for example phosphatidylserine in the outer leaflet of the membrane, changes in pattern of glycosylation of cell surface proteins and surface charge $[155,156]$. Several receptors were found to act in the phagocytosis of apoptotic cells. Those include scavenger-receptor-AI/II [157], class B scavenger R (CD36), vitronectin receptor [158] and CD14 [159]. Scavenger receptor A binds acetylated and oxidized low-density lipoprotein [160]. CD36 has been identified as one of the receptors for collagen type I, thrombospondin, oxidized LDL and phosphatidylserine [161].

In addition, the Fc receptor and the complement receptor are involved in phagocytosis [162]. The type I phagocytosis [163, 164] initiated by the Fc-receptor via immune IgG binding is characterized by extension of pseudopodia and envelopment of the phagocytic target. This mechanism relies on the activity of tyrosine kinases and small G-proteins Rac and Cdc42. In contrast, type II phagocytosis results from ligation of CR3/ Mac-1 by complement opsonized particles [163]. Complement proteins, present in serum, opsonize bacteria for phagocytosis [165]. This mechanism depends on the activation of RhoA and requires microtubules $[163,164,166]$.

Particle internalization is initiated by the interaction of specific surface receptors of the phagocyte with ligands on the surface of a particle, leading to the polymerization of actin at the site of ingestion. The particle is internalized via an actin-based mechanism, and finally actin is removed from the phagosome and maturation culminates in the phagolysosome [167]. Besides PI-3 kinase, the family of RhoGTPases, protein kinase $\mathrm{C}$ and also motor proteins appear to participate in the stimulation of actin polymerization and induce the formation of phagosomes [168, 169]. Rho family 
GTPases cycle between an inactive GDP bound state and the active GTP-bound state whilst their activation is mediated by specific guanine-nucleotide-exchange factors (GEFs). Inhibition of Rac1 and Cdc42 blocks phagocytosis by preventing the accumulation of F-actin in the phagocytic cup [170]. ARF6 inhibition, implicated in endocytosis and membrane recycling, blocks Fc $\gamma$ R-mediated phagocytosis in macrophages [171]. Often antibody-mediated blocking of specific receptors or genetic ablation of a single receptor have only a partial effect on the engulfment of cell debris. Apoptotic cell recognition appears to be dependent on the target cell, the set of receptors expressed by the phagocyte and the state of activation of the phagocyte during engulfment $[172,173]$.

\subsubsection{Macropinocytosis}

Unlike receptor-mediated endocytosis and phagocytosis, macropinocytosis seems not to be regulated by the direct actions of cargo/receptor molecules [174]. Hence, it represents a distinct pathway of endocytosis in mammalian cells. Macropinocytosis is usually initiated by external stimulation by growth factors and involves the formation of large vacuoles [175]. It is primarily used for the non-selective internalization of fluid and membrane [176]. However, it is also used by pathogens like bacteria and viruses for the specific infectious entry of the cell [177-180].

Macropinocytosis is associated with membrane ruffling characterized by circular cup-shaped membrane extensions and large plasma membrane extrusions [175]. This process is thought to be activated by growth factors triggering activation of receptor tyrosine kinases, which in turn activate a signaling cascade regulating dynamics of actin filaments. Actin and many of its key regulators are therefore associated with macropinocytic activity $[181,182]$. While most ruffles fuse back into the plasma membrane, a few fold back forming fluid filled cavities and undergo membrane fission to become macropinosomes [175]. Macropinosomes are inhomogeneous in size with a diameter of $0.5-10 \mu \mathrm{m}[175]$ and share many features with the endosomal/lysosomal system. In macrophages it was shown that macropinosomes migrate in a centripetal manner and rapidly acquire markers of the late endosome, such as Rab7, before 
ultimately fusing with the lysosomal system [183]. Macrophages as well as DCs are capable of both macropinocytosis and phagocytosis [184].

While immature DCs actively carry out macropinocytosis and phagocytosis, in mature DCs these activities are less pronounced [185-187]. Actin and its regulation by Rho family GTPases play important roles in mediating phagocytosis and macropinocytosis. Especially, levels of activated Cdc42 are used to regulate their endocytic capacity during development [188].

\subsection{Aims and objectives}

Oligodendrocytes as the myelin forming cells of the central nervous system are specialized to synthesize large amounts of membrane. These cells secrete membrane vesicles, so-called exosomes, into the extracellular space that are enriched in the proteolipid protein, a major myelin protein and a candidate autoantigen in multiple sclerosis. The goal of this study was to analyze the fate and function of oligodendroglial exosomes in the extracellular milieu.

One putative function of oligodendroglial exosomes is to remove obsolete proteins and lipids during oligodendroglial membrane production and turn-over. Oligodendrocytes might not have the capacity to entirely recycle myelin components, but instead employ microglia for this task. Moreover, oligodendroglial exosomes can also represent putative "antigen shuttles" during autoimmune diseases. To study the fate of oligodendroglial exosomes, we sought to address the following main questions: How are exosomes internalized and by which cells? Do exosomes transfer antigens to antigen-presenting cells?

To reveal the function of exosomes, we used purified exosomes both from oligodendroglial precursor cell line as well as primary cells. We analyzed their fate in microglial cell lines, primary glial culture systems and transgenic mice. We assessed their internalization trough visualization by confocal and two-photon microscopy, as well as with biochemical and ELISA based approaches. 


\section{Chapter 2}

\section{Materials \& methods}

\subsection{Materials}

\subsubsection{Chemicals and consumables}

All chemicals used in this study were obtained from Sigma-Aldrich (Sigma-Aldrich Chemie GmbH, Munich, Germany), Merck (Merck KGaA, Darmstadt, Germany), or AppliChem (AppliChem GmbH, Darmstadt, Germany), unless stated otherwise.

All basal media, supplements, antibiotics and sera for cell culture were purchased from Gibco/Invitrogen (Invitrogen GmbH, Darmstadt, Germany) or PAA (PAA Laboratories GmbH, Cölbe, Germany).

Consumables were purchased from Falcon (Becton Dickinson Labware Europe, Le Pont De Claix, France) and Eppendorf (Eppendorf AG, Hamburg, Germany). CELLSTAR culture vessels were obtained from Greiner Bio-One (Greiner Bio-One GmbH, Frickenhausen, Germany). Ultracentrifugation tubes were obtained from Beckman (Beckman Coulter GmbH, Krefeld, Germany).

\subsubsection{Antibodies and fluorophore-coupled compounds}

Primary antibodies used in this study are listed in Table 2.1. Rhodamine-conjugated wheat germ agglutinin (WGA) used for general membrane staining was obtained from Molecular probes (Invitrogen, Carlsbad, CA, USA).

Annexin V-FITC and Isolectin B4-FITC were purchased from Sigma-Aldrich (SigmaAldrich Chemie GmbH, Munich, Germany). FITC-dextran and Rhodamine-dextran 
(MW>40,000) was obtained from Molecular Probes (Invitrogen, Carlsbad, CA, USA). Secondary fluorophore- and peroxidase-conjugated antibodies were purchased from Dianova (Hamburg, Germany).

Table 2.1: Antibodies

\begin{tabular}{llll}
\hline Target & Host species & Application & Reference \\
\hline B7-1 & Mouse monoclonal & IF $(1: 50)$ & Pharmingen, BD , San Jose, CA, USA \\
B7-2 & Mouse monoclonal & IF $(1: 15)$ & Pharmingen, BD , San Jose, CA, USA \\
Calnexin & Rabbit & WB $(1: 1000)$ & Stressgen Bioreag., Victoria, Canada \\
GFAP & Rabbit & IF $(1: 100)$, WB (1:1000) & Promega, Mannheim, Germany \\
GM130 (cis-Golgi) & Mouse IgG1 & IF $(1: 200)$, WB (1:500) & BD Transd. Lab., San Jose, CA, USA \\
Iba1 & Rabbit & IF $(1: 200)$, WB (1:2000) & Wako Chem. GmbH, Neuss, Germany \\
Lamp-1 & Rat IgG2a & IF $(1: 200)$ & BD Biosciences, San Jose, CA, USA \\
MHCII & Rat & IF $(1: 200)$ & clone M5/114.15.2 \\
MOG (clone 8-18-C5) & Mouse IgG1 & IF $(1: 100)$, WB (1:500) & Millipore, Billerica, MA, USA \\
Myc (clone 9E10) & Mouse IgG1 & IF $(1: 400)$ & Sigma-Aldrich, Munich, Germany \\
O1 & Mouse IgM & IF $(1: 50)$ & [189, 190] \\
PLP (clone 3F4) & Mouse IgG & WB (1:100) & [191] \\
Synaptophysin 1 & Mouse IgG & WB (1:1000) & Synaptic Sys., Göttingen, Germany \\
Tubulin, $\beta$ III & Mouse IgG1 & IF $(1: 3000)$ & Promega, Mannheim, Germany \\
\hline
\end{tabular}

\subsubsection{DNA constructs}

The following plasmids were used in this study:

- pcDNA3.1-MOG (full length) [192]

- and pEGFP-PLP-myc [193]

Plasmid DNA was amplified in DH5 $\alpha E$. coli strains (Subcloning Efficiency DH5 $\alpha$, Invitrogen, Carlsbad, CA, USA) and purified using commercial kits listed in Table 2.3. 


\subsubsection{Buffers and solutions}

$10 \times$ PBS was prepared according to [194]:

\section{X PBS (1€)}

$80.0 \mathrm{~g} \mathrm{NaCl}$

$2.0 \mathrm{~g} \quad \mathrm{KCl}$

$14.4 \mathrm{~g} \quad \mathrm{Na}_{2} \mathrm{HPO}_{4}\left(\right.$ or $\left.18.05 \mathrm{~g} \mathrm{Na}_{2} \mathrm{HPO}_{4} \times 2 \mathrm{H}_{2} \mathrm{O}\right)$

$2.4 \mathrm{~g} \quad \mathrm{KH}_{2} \mathrm{PO}_{4}$

To obtain $1 \mathrm{X}$ PBS, 10X PBS was diluted 10 times with $\mathrm{ddH}_{2} \mathrm{O}$, pH value was adjusted to 7.4 .

\subsubsection{Cell culture media}

\section{Super SATO Medium}

Primary oligodendroglial cultures used in this study were grown in Super SATO medium [193]:

\section{Super SATO medium $(100 \mathrm{~mL})$}

$2 \mathrm{~mL}$ B-27 Supplement, 50X

$1 \mathrm{~mL}$ GlutaMAX-1 supplement, $200 \mathrm{mM}$

$1 \mathrm{~mL}$ Penicillin/Streptomycin, $5000 \mathrm{U} / 5000 \mu \mathrm{g}$

$1 \mathrm{~mL}$ Sodium pyruvate, $100 \mathrm{mM}$

$10 \mu \mathrm{L}$ Triiodothyronine (Calbiochem/Merck KGaA, Darmstadt, Germany), $5 \mathrm{mM}$ stock in ethanol

$13 \mu \mathrm{L}$ L-Thyroxine (Calbiochem/Merck KGaA, Darmstadt, Germany), $4 \mathrm{mM}$ stock in $0.26 \mathrm{~N} \mathrm{NaOH}, 25 \%$ ethanol

$1 \mathrm{~mL}$ Horse serum (PAA Laboratories GmbH, Pasching, Austria) in Dulbecco's Modified Eagle Medium (DMEM) with $4.5 \mathrm{~g} / \mathrm{L}$ glucose 


\section{SATO Medium}

The oligodendroglial precursor cell line Oli-neu [195] was grown in SATO medium [193]:

\section{SATO medium $(100 \mathrm{ml})$}

$1 \mathrm{~mL}$ Insulin-Transferrin-Selenium-A Supplement ITS-A, 100X

$1 \mathrm{~mL}$ Putrescine dihydrochloride, stock $10 \mathrm{mM}$ in DMEM

$10 \mu \mathrm{L}$ Progesterone, stock $2 \mathrm{mM}$ in ethanol

$10 \mu \mathrm{L}$ Triiodothyronine ( Calbiochem/Merck KGaA, Darmstadt, Germany), $5 \mathrm{mM}$ stock in ethanol

$13 \mu \mathrm{L}$ L-Thyroxine (Calbiochem/Merck KGaA, Darmstadt, Germany), $4 \mathrm{mM}$ stock in $0.26 \mathrm{~N} \mathrm{NaOH}, 25 \%$ ethanol

$1 \mathrm{~mL}$ GlutaMAX-1 supplement, $200 \mathrm{mM}$

$1 \mathrm{~mL}$ Penicillin/Streptomycin, $5000 \mathrm{U} / 5000 \mu \mathrm{g}$

$5 \mathrm{~mL}$ Horse serum (PAA Laboratories GmbH, Pasching, Austria)

in Dulbecco's Modified Eagle Medium (DMEM) with $4.5 \mathrm{~g} / \mathrm{L}$ glucose.

\subsubsection{Inhibitors, sugars and peptides for cell culture assays}

All inhibitors, sugars, proteins and peptides used for various cell culture experiments (see Table 2.2) were purchased from Sigma-Aldrich (Sigma-Aldrich Chemie GmbH, Munich, Germany) or Calbiochem (Calbiochem/Merck KGaA, Darmstadt, Germany). LDL-receptor-related protein associated protein 1 (LRPAP1) was obtained from Enzo lifescience (Enzo Life Sciences International, Inc, PA, USA) and recombinant mouse MFGE8 protein was purchased from RD Systems.

\subsubsection{Commercial kits}

Commercial kits used in this project are listed in Table 2.3.

\subsubsection{Specific software}

List of specific software used for assay design, data acquisition, processing and analysis can be found in Table 2.4 . 
Table 2.2: Inhibitors, sugars and peptides

\begin{tabular}{ll}
\hline Inhibitors, Sugars and Peptides & Application (final conc.) \\
\hline Amiloride & $1 \mathrm{mM}$ \\
Bafilomycin A1 & $10 \mathrm{nM}$ \\
Blebbistatin & $50 \mu \mathrm{M}$ \\
Chloroquine & $20 \mu \mathrm{M}$ \\
Cytocholasin D & $2 \mu \mathrm{M}$ \\
Dynasore & $80 \mu \mathrm{M}$ \\
Eppidermal gowth factor (EGF) & $10 \mathrm{ng}$ to $100 \mathrm{ng} / \mathrm{mL}$ \\
Fucoidan & $40 \mathrm{ng} / \mathrm{mL}$ \\
Monensin & $14 \mu \mathrm{M}$ \\
rMFGE8 & $100 \mathrm{ng} / \mathrm{mL}$ \\
N-acetyl-D-glucosamine & $20 \mathrm{mM}$ \\
D-mannosamine-hydrochloride & $20 \mathrm{mM}$ \\
G-RGD-SP peptide (RGD) & $1 \mathrm{mg} / \mathrm{mL}$ \\
G-RAD-SP peptide (RAD) & $1 \mathrm{mg} / \mathrm{mL}$ \\
NSC23766 & $300 \mu \mathrm{M}$ \\
LRPAP1 (RAP) & $250 \mathrm{to} 500 \mathrm{nM}$ \\
Interferon $\gamma($ IFN $\gamma$ ) & $100 \mathrm{ng} / \mathrm{ml}$ \\
Lipopolysaccharide (LPS) & $1 \mathrm{ng}$ to $10 \mathrm{ng} / \mathrm{mL}$ \\
&
\end{tabular}

\subsection{Methods}

\subsubsection{Biochemistry}

\section{Preparation of cell lysates}

Primary glial cells or Oli-neu cells were cultured in culture dishes (see Section 2.2.2). The cell culture medium was removed, used for exosomes purification (see Section 2.2.4) or discarded. The cell layer was washed once in PBS and scraped on ice into $100 \mu \mathrm{L}$ of lysis buffer (2\% NP-40, $0.2 \%$ SDS, 0.5 mM EDTA in PBS) supple- 
Table 2.3: Commercial kits

\begin{tabular}{lll}
\hline Commercial kit & Application & Manufacturer \\
\hline BCA & Protein Concentration Measurements & Thermo Scientific, Rockford, IL, USA \\
FITC Antibody Labeling kit & Labeling of Myelin & Thermo Scientific, Rockford, IL, USA \\
NucleoBond Xtra Midi kit & DNA isolation, medium scale & Macherey-Nagel, Dueren, Germany \\
NucleoSpin Plasmid kit & DNA isolation, small scale & Macherey-Nagel, Dueren, Germany \\
S $^{3} 5$ Protein Labelling Mix & Metabolic Labeling of Proteins & Perkin Elmer LifeScience, MA, USA \\
QIAprep Spin Miniprep kit & DNA isolation, small scale & Qiagen, Hilden, Germany \\
\hline
\end{tabular}

Table 2.4: List of software

\begin{tabular}{lll}
\hline Software & Application & Source/Manufacturer \\
\hline ImageJ & Image processing/analysis & http://rsbweb.nih.gov/ij/ \\
Leica Confocal Software, 2.61 & Acquisition confocal images & Leica, Mannheim, Germany \\
Matlab Version 7 & Analysis of Two-Photon LSM Data & MathWorks, Ismaning, Germany \\
Meta Imaging Series 6.1 & Image processing and analysis & Universal Imaging Corp., USA \\
WinMDI software & Analysis of flow cytometric data & Purdue University, USA \\
Statistica & Statistical analysis & StatSoft GmbH, Hamburg, Germany \\
Zeiss LSM software & Acquisition confocal images & Zeiss, Jena, Germany \\
\hline
\end{tabular}

mented 1:50 with protease inhibitor ${ }^{1}$. The cell lysate was either directly used for Western Blot analysis or stored at $-20^{\circ} \mathrm{C}$.

\section{Determination of protein concentration}

The protein concentration of cell lysates was determined using Pierce BCA Protein Assay (Thermo Scientific, Rockford, IL, USA) according to the manufacturer's instructions. This protein quantitation assay is based on bicinchoninic acid (BCA) and combines a two step detection process. In the first step $\mathrm{Cu}(+\mathrm{II})$ ions are reduced by the introduced protein to $\mathrm{Cu}(+\mathrm{I})$ in an alkaline medium. In the second step, BCA reacts with the monovalent cuprous cation allowing the colorimetric detection [196]. The absorbance of the purple-colored reaction product was measured at $562 \mathrm{~nm}$ using a 96-well plate reader (MRXTc Revelation, Dynex Technologies).

\footnotetext{
${ }^{1}$ Protease inhibitors cocktail Complete Mini (Roche Applied Science, Mannheim, Germany). Stock: 1 tablet in $1 \mathrm{~mL}$ PBS.
} 
Sodium dodecyl sulfate polyacrylamide gel electrophoresis (SDS-PAGE)

The electrophoretic separation of proteins was carried out in polyacrylamide gels. Crucial for the accuracy of this process is the ionic detergent sodium dodecyl sulfate (SDS), which is necessary to denature proteins before and during the gel electrophoresis. SDS binds to the polypeptide backbone of all proteins and covers intrinsic charges of the amino acid chain. For this reason, proteins lose their secondary and tertiary structures and can be separated according to their size. In addition, $\beta$-mercaptoethanol can be added, which further denatures the proteins by reducing disulfide linkages, thus overcoming some forms of tertiary protein folding, and breaking up the quaternary protein structure [197].

Before loading on the gel, the samples were incubated in reducing sample buffer at $95^{\circ} \mathrm{C}$ for $5 \mathrm{~min}$ (composition see below). Exceptions were applied for MOG protein analysis with semi-reducing SDS-PAGE conditions (incubation at $60^{\circ} \mathrm{C}$ for $10 \mathrm{~min}$ in sample buffer without $\beta$-mercaptoethanol) and for PLP detection (incubation in sample buffer was performed at $55^{\circ} \mathrm{C}$ for $10 \mathrm{~min}$.).

\section{Sample buffer for SDS-PAGE}

$50 \mathrm{mM}$ Tris/HCl pH 6.8

$10 \% \quad$ Glycerol

$2 \mathrm{mM} \quad$ EDTA

$2 \% \quad$ SDS

0.05\% Bromophenol blue

$144 \mathrm{mM} \quad \beta$-mercaptoethanol (approx. 2\% v/v)

The SDS-gels were prepared in a Bio-Rad Mini-PROTEAN 3 casting system, separation of proteins was carried out in vertical electrophoresis systems Bio-Rad Mini-PROTEAN (Bio-Rad Laboratories GmbH, Munich, Germany) in Tris-glycine electrophoresis buffer (25 mM Tris, $192 \mathrm{mM}$ glycine, 0.1\% SDS) for $2 \mathrm{~h}$ at $100 \mathrm{~V}$ $(15 \mathrm{~V} / \mathrm{cm})$. Resolving gel and stacking gels were of the following composition: 
Stacking gel

4\% Acrylamide/bis-acrylamide 29:1 solution

$125 \mathrm{mM} \quad$ Tris/HCl, $\mathrm{pH} 6.8$

$0.1 \% \quad$ SDS

0.05\% Ammonium persulfate (APS)

$0.005 \%$ (v/v) N'N'N'N'-tetramethylethylene-1,2-diamine (TEMED)

\section{Resolving gel (8 - 15\%)}

8 to $15 \% \quad$ Acrylamide/bis-acrylamide 29:1 solution

$375 \mathrm{mM} \quad$ Tris/HCl, $\mathrm{pH} 8.8$

$0.1 \% \quad$ SDS

$0.05 \% \quad$ APS

$0.005 \%(\mathrm{v} / \mathrm{v})$ TEMED

Molecular weights of the analyzed proteins were estimated according to protein molecular weight marker PageRuler@ Plus Prestained Protein Ladder (Fermentas, St. Leon-Rot, Germany).

\section{Western blotting}

The Western blotting technique first described in 1979, is used for the identification and quantification of electrophoretically separated proteins [198]. Upon separation in SDS-polyacrylamide gels, proteins were transferred by electroblotting from the gel onto a Whatman@ Protran Nitrocellulose Transfer Membrane (Whatman GmbH, Dassel, Germany). Protein transfer was carried out for $1 \mathrm{~h}$ at $100 \mathrm{~V}$ in a transfer buffer (25 mM Tris, $192 \mathrm{mM}$ glycine, 20\% methanol) using the Bio-Rad Mini-Protein System (Bio-Rad Laboratories GmbH, Munich, Germany). Afterwards, proteins were detected using antibodies specific to the target proteins.

For immune detection, membranes were transferred into $4 \%$ nonfat dried milk (Sigma-Aldrich Chemie GmbH, Munich, Germany) in PBS for 30 min at room temperature (RT), to reduce unspecific binding of immunoglobulins. Incubation with 
primary antibodies was carried out in PBS supplemented with $0.1 \%$ (v/v) Tween20 (PBST) for $1 \mathrm{~h}$ at RT or for $16 \mathrm{~h}$ at $4^{\circ} \mathrm{C}$ (see Section 2.1.2 for the appropiate antibody dilution). After three 10 min washing steps with PBST, membranes were incubated with the appropiate secondary antibodies conjugated to horseradish peroxidase (HRP) (dilution 1:2000; purchased from Dianova, Hamburg, Germany). After incubation of $1 \mathrm{~h}$ at RT, the membrane was washed three times for $10 \mathrm{~min}$ with PBST.

Finally, antigens were visualized by chemiluminescence using the Pierce ECL Western Blotting Substrate from Thermo Scientific (Epsom, United Kingdom). X-ray films (CL-XPosure ${ }^{\mathrm{TM}}$ Film, ThermoScientific, Rockford, IL, USA) were exposed to chemiluminescent signals and developed. Afterwards, signals were scanned with a conventional scanner and the signal densities were quantified with ImageJ ${ }^{2}$.

\subsubsection{Cell culture techniques}

Primary cell cultures and cell lines were maintained in humidified $37^{\circ} \mathrm{C}, 7.5 \% \mathrm{CO}_{2}$ incubators; further culture conditions are specified below. All media and used compounds (see Section 2.1.5, Section 2.1.6) were sterile or sterilized using $0.22 \mu \mathrm{m}$ polyethersulfone (PES) filters (Corning Inc., Corning, NY, USA). Cell culture vessels and coverslips for primary cultures were coated with poly-L-lysine ( $\mathrm{Mw}>300000)$. Briefly, $100 \mu \mathrm{g} / \mathrm{mL}$ PLL was applied on the surfaces for 4 to $12 \mathrm{~h}$ at $37^{\circ} \mathrm{C}$, aspirated and washed with PBS. For cell lines $33 \mu \mathrm{g} / \mathrm{mL}$ PLL was applied on the surfaces for 30 min at RT, followed by washing with PBS respectively.

\section{Primary glial culture}

Primary glial cultures were prepared as described previously [193]. Briefly, brains were extracted of NMRI mice (postnatal day 1) and meninges were removed. After trypsinization for $10 \mathrm{~min}$, cells were mechanically separated by pipetting and seeded into cell culture flasks. Cells were grown in Basal Medium Eagle (BME) medium supplemented with 10\% horse serum (PAA Laboratories GmbH, Pasching, Austria) and $1 \%$ penicillin/streptomycin.

\footnotetext{
${ }^{2}$ http://rsbweb.nih.gov/ij/
} 
After two weeks of incubation primary oligodendrocyte progenitors were shaken of the mixed glial culture and plated onto poly-L-lysine (PLL) coated coverslips or dishes. Primary oligodendrocyte progenitors were kept for $5 \mathrm{~d}$ in Super SATO medium (see Section 2.1.5) to allow their differentiation.

The remaining cell monolayer comprised of microglia and astrocytes was further incubated in DMEM with 10\% FCS and microglia proliferation was stimulated by addition of 15\% MCSF-enriched cell culture supernatant from L929 cells (see Section 2.2.2). After four days, microglia were shaken off and plated in dishes or onto coverslips.

Astrocyte cultures were prepared by trypsinization of mixed glial cultures after oligodendrocytes and microglia were shaken off and were maintained in Dulbecco's modified Eagle's medium (DMEM) with 10\% fetal calf serum (FCS)and $1 \%$ penicillin/streptomycin.

\section{Primary neuronal culture}

For primary neuronal cultures brains of fetal NMRI mice (embryonic day E15 - E17) were prepared, meninges removed and hemispheres collected. After trypzinization for $5 \mathrm{~min}$, cells were mechanically separated by pipetting and seeded onto PLLcoated coverslips in Super SATO medium (see Section 2.1.5 at a density of 70,000 to 150,000 cells per coverslip. Neuronal cultures were grown for $14 \mathrm{~d}$ before being used for experiments [193, 199].

\section{Growth and maintenance of EOC-20 cell line}

The mouse microglial cell line EOC-20 (kindly provided by H. Ehrenreich, MPI for Experimental Medicine, Göttingen, Germany) was cultured in DMEM with $10 \%$ FCS, $1 \%$ pyruvate, $1 \%$ L-glutamate, $1 \%$ penicillin/streptomycin and $100 \mathrm{ng} / \mathrm{ml}$ mouse recombinant macrophage colony stimulating factor (MCSF, Sigma-Aldrich Chemie GmbH, Munich, Germany). Growth medium was changed every three to four days and cells were split once a week (1:3), when grown to a confluency of $80 \%$. 


\section{Growth and maintenance of L929 cell line}

The mouse fibroblast cell line L929 (kindly provided by U.-K. Hanisch, Institute for Neuropathology, University of Göttingen, Germany) was cultured in DMEM with $10 \%$ FCS for two weeks to allow secretion of macrophage colony stimulating factor (MCSF). The conditioned medium was used for stimulation of primary microglial cultures (see above). The L929 cells were split every two weeks by trypsinization and seeded (1:5) in cell culture flasks.

\section{Growth and maintenance of Oli-neu cell line}

The mouse oligodendrocyte precursor cell line Oli-neu ([195]; kindly provided by J. Trotter, Heidelberg, Germany) was cultured in SATO medium (see Section 2.1.5) containing $5 \%$ horse serum on poly-L-lysine (PLL) coated coverslips or cell culture dishes. The cells were passaged 1:5 - 1:6 every two to three days when grown to approx. $80 \%$ confluence.

\subsubsection{Transfection of mammalian cells}

The TransIT® transfection reagent (Mirus Bio LLC, Madison, WI, USA) was used to introduce expression vectors into cultured Oli-neu cells. This system takes advantage of liposomes, which are capable of encapsulating or binding nucleic acids, to fuse with cellular membranes and thus delivering exogenous DNA into cells [200]. For the transfection, Oli-neu cells were cultured to approx. $75 \%$ confluence on the day of transfection. For each coverslip in a 12-well plate, $3 \mu \mathrm{L}$ of transfection reagent and $1 \mu \mathrm{g}$ of plasmid DNA were added subsequently to $100 \mu \mathrm{L}$ OptiMEM-I (Invitrogen, Carlsbad, CA, USA) and gently mixed. After incubation at RT for 30 min, the dilution was added dropwisly to the cell culture. Afterwards, cells were incubated for 8 to $16 \mathrm{~h}$ prior to the experiment. For the transfection of cells grown in $10 \mathrm{~cm}$ dishes, $15 \mu \mathrm{L}$ of TransITR and $5 \mu \mathrm{g}$ plasmid DNA were used accordingly. 


\subsubsection{Exosome purification and labeling}

All ultracentrifugation steps were carried out in a fixed angle 45Ti rotor using a Beckman XL-70 Ultracentrifuge (Beckman Coulter GmbH, Krefeld, Germany), unless otherwise stated.

\section{Exosome purification}

Exosomes from Oli-neu cells and primary oligodendrocytes were purified as described previously $[12,13]$. One day prior to the preparation of exosomes, the cell culture medium was replaced by the respective serum-free medium to exclude contamination with serum-derived exosomes. Cell culture supernatants were collected after 12 to $24 \mathrm{~h}$ of incubation. The medium was centrifuged once at $3,000 \times \mathrm{g}$ and twice at $4,000 \times \mathrm{g}$ for $10 \mathrm{~min}$, followed by $10,000 \times \mathrm{g}$ for $30 \mathrm{~min}$ to remove dead cells and cell debris. Finally, exosomes were pelleted by ultracentrifugation at $100,000 \times$ g for $1 \mathrm{~h}$. The exosomal pellet was resuspended in PBS or in sample buffer for Western blot analysis. The isolation and analysis of primary rat oligodendrocytes was performed by Mostafa Bakhti, Max Planck Institute for Experimental Medicine, 37075 Göttingen, Germany .

\section{Analysis of exosomes on continuous sucrose gradients}

To analyze the purity of the $100,000 \times \mathrm{g}$ pellet, a continuous sucrose gradient centrifugation was applied. This technique allows the separation of membrane vesicles according to their density [13]. For the analysis, sucrose solutions of different densities (1.03 to $1.32 \mathrm{~g} / \mathrm{cm}^{3}$ sucrose in $10 \mathrm{mM}$ Hepes) were carefully overlayed in an ultracentrifugation tube. The $100,000 \times \mathrm{g}$ pellet suspension was added on top of the gradient and centrifuged for $16 \mathrm{~h}$ at $200,000 \times \mathrm{g}$. This centrifugation step was carried out in swinging bucket rotor necessary for the establishment of a continuous sucrose gradient (Sw41-Ti, Beckman Coulter GmbH, Krefeld, Germany). Subsequently, eight fractions were recovered, diluted 1:3 in PBS and centrifuged at 100,000 $\times$ g for $1 \mathrm{~h}$ to allow sedimentation of all comprised membrane vesicles. Pellets of all fractions were resuspended in PBS and subjected to Western blot analysis or to exosome internalization assay experiments. 


\section{Fluorescent labeling of exosomes}

For immunofluorescence analysis, the exosomal pellet was resuspended in PBS and exosomes were stained with the lipophilic dye PKH67 or PKH26, respectively (Fluorescent Cell Linker Kits, Sigma-Aldrich Chemie GmbH, Munich, Germany) [201]. These fluorescent dyes contain long aliphatic tails allowing stably integration into lipid regions of the cellular membranes [202]. For the staining reaction, $100 \mu \mathrm{L}$ exosome suspension was mixed with $900 \mu \mathrm{L}$ of DiluentC and $1 \mu \mathrm{L}$ of dye. The reaction was stopped after $5 \mathrm{~min}$ of incubation with $200 \mu \mathrm{L}$ exosome-free FCS. Exosomes were washed with PBS to remove unbound dye and pelleted by ultracentrifugation $(100,000 \times \mathrm{g}, 1 \mathrm{~h})$.

Alternatively, exosomes were labeled by cross linking of carboxytetramethylrhodamine succinimidyl ester (TAMRA-NHS, Biotinum, Inc.) to exosomal proteins as described previously [203]. Briefly, $100 \mu \mathrm{L}$ of exosome suspension was added to $1 \mathrm{~mL}$ of $0.1 \mathrm{M}$ sodium bicarbonate buffer ( $\mathrm{pH} 8.3$ ) containing $100 \mu \mathrm{g}$ TAMRA-NHS. After $1 \mathrm{~h}$ of incubation, unincorporated TAMRA-NHS was removed using a $100 \mathrm{kDa}$ ultrafiltration tube (Amicon Ultra, Millipore). Exosomes were washed once with PBS and pelleted by ultracentrifugation $(100,000 \times \mathrm{g}, 1 \mathrm{~h})$.

\subsubsection{Exosome internalization assay}

\section{In vitro exosome uptake assay}

For the exosome internalization assay, primary glial or neuronal cells were plated on coverslips and incubated for one to seven days in the respective growth media. Prior to the uptake assay, exosomes were purified, labeled and diluted in growth medium (see Section 2.2.4). The uptake was performed by incubating cell cultures with exosome dilutions in a humid chamber for $2 \mathrm{~h}\left(37^{\circ} \mathrm{C}, 7 \% \mathrm{CO}_{2}\right)$.

For inhibition experiments, cell cultures were pre-incubated with the respective compound for 30 min before performing the uptake in its further presence (see Table 2.2). For liposome experiments, liposomes were mixed with exosomes in the respective growth medium prior to the experiment (see below). Subsequently, cells were fixed with $4 \%$ PFA and stained for 15 min with the membrane dye WGA or subjected to immunocytochemistry, respectively (see Section 2.1.2). 
For the quantification of internalization assays, ten to twenty viewing field images of each sample were randomly captured on a Leica DMRXA microscope, with a $40 \times$ oil objective (Leica, Nussloch, Germany). Integrated fluorescence intensity was determined with a module of Meta Imaging Series 6.1 software (Universal Imaging Corporation, West Chester, PA, USA). A fixed threshold was used to eliminate the low-intensity background signal in both channels. Integrated fluorescence intensity of exosome signals was normalized with the respective integrated WGA surface stain intensity. For correlation of MHC class II staining with exosome uptake, intensities of single cells were measured and compared. Normal distribution of the data was analyzed with Kolmogorov-Smirnov-test and significance was tested using Student's t-test or Mann-Whitney-U test, respectively. Results were regarded as significantly different if p-values below 0.05 were reached. The level of significance was indicated as follows: ${ }^{*} \mathrm{p}<0.05,{ }^{* *} \mathrm{p}<0.01$ and ${ }^{* * *} \mathrm{p}<0.001$. Parts of the exosome internalization experiments were performed by D. Fitzner, Department of Neurology, University of Göttingen/ Max Planck Institute for Experimental Medicine, 37075 Göttingen, Germany.

\section{Liposome preparation}

Liposomes used for the exosome internalization assay were prepared as described previously [204]. For internalization assays, the phospholipids L- $\alpha$-phosphatidylcholine (PC) and L- $\alpha$-phosphatidylserine (PS) (Avanti Polar Lipids) were either used separately or in a $50: 50$ ratio. Briefly, liposomes were prepared by resuspension of phospholipids in HBS (100 mM NaCl, 20 mM Hepes/NaOH buffer, pH 7.5). Subsequently, vesicles were repeatedly passed through a polycarbonate filter to obtain uniformly sized, unilamellar vesicles of approximately $100 \mathrm{~nm}$ in diameter. For internalization assays exosomes from Oli-neu cells were mixed with liposomes containing either PC or PC/PS in a final concentration of $100 \mu \mathrm{M}$ (see above).

\section{Live imaging of exosome internalization}

For live imaging of exosome uptake, coverslips containing EOC-20 cells were placed in a self-made closed imaging chamber with imaging medium containing PKH26- 
labeled exosomes (HBSS, 10mM HEPES, 1\% horse serum, pH 7.4, 37 ${ }^{\circ} \mathrm{C}$ ). Imaging was performed in a tempered live cell imaging chamber (The cube/The box, Live Imaging Services) and observed using a Leica confocal microscope DMIRE2. Images were required at $2 \mathrm{~s}$ intervals using sequential line excitation at 488 and $543 \mathrm{~nm}$ and appropriate band pass emission filters. The pinhole was set to 1.12 airy units. Live Imaging of exosome internalization was performed by D. Fitzner, Department of Neurology, University of Göttingen/ Max Planck Institute for Experimental Medicine, D-37075 Göttingen, Germany.

\subsection{6 $S^{35}$ Metabolic labeling of proteins}

The quantitative analysis of oligodendroglial exosome release was analyzed using a radioactive $S^{35}$ pulse-chase approach. Cellular proteins were randomly labeled by a pulse of $\mathrm{S}^{35}$-methionine/ $\mathrm{S}^{35}$-cysteine and the amount of radioactivity released via the exosomal fraction was determined over time.

For the metabolic labeling, 70,000 primary oligodendroctes or 80,000 Oli-neu cells were seeded into 12-well cavities and grown in the appropriate culture medium (see Section 2.1.5). The next day, medium was changed to methionine/cysteine-free growth medium for $30 \mathrm{~min}$. Afterwards, medium was supplemented with $165 \mu \mathrm{Ci}$ per cavity of $\mathrm{S}^{35}$ protein labeling mix (Perkin Elmer Life Science, MA, USA). After incubation for $1 \mathrm{~h}$, cells were washed three times with methionine/cysteine-free growth medium and exosome collection medium was added (horse serum-free growth medium supplemented with $2 \mathrm{mM}$ cysteine/ methionine). Medium and cell lysates were collected after 0,16 and $24 \mathrm{~h}$ of chase and supernatants were subjected to exosome purification. Radioactive decay of samples was measured using a scintillation counter (Beckmann LS6000LL; Beckman Coulter GmbH, Krefeld, Germany).

\subsubsection{Myelin preparation and internalization assay}

For the isolation of myelin, all ultracentrifugation steps were carried out in a swinging bucket Sw41-Ti rotor using a Beckman XL-70 Ultracentrifuge (Beckman Coulter GmbH, Krefeld, Germany). 


\section{Myelin purification on discontinuous sucrose gradients}

Brains from six weeks old wild-type NMRI mice were prepared and washed once in PBS. Each brain was homogenized by sonication in $1 \mathrm{~mL}$ ice-cold PBS supplemented 1: 50 with protease inhibitors (Protease inhibitors cocktail Complete Mini, Roche Applied Science, Mannheim, Germany; Stock: 1 tablet in $1 \mathrm{~mL}$ PBS). The homogenate was resuspended in $10 \mathrm{~mL}$ of $0.32 \mathrm{M}$ sucrose solution (5 mM EDTA, $10 \mathrm{mM}$ HEPES, pH 7.4). Afterwards, $5 \mathrm{~mL}$ of the homogenate at a time were layered over $5 \mathrm{~mL}$ of $0.85 \mathrm{M}$ sucrose solution ( $5 \mathrm{mM}$ EDTA, $10 \mathrm{mM}$ HEPES, pH 7.4) and the sample was spun at $75,000 \times \mathrm{g}$ for $30 \mathrm{~min}$. After centrifugation, the interface between $0.32 \mathrm{M}$ and $0.85 \mathrm{M}$ sucrose was collected, diluted at least 10 times with ice-cold $\mathrm{H}_{2} \mathrm{O}$ and centrifuged again at $75,000 \times \mathrm{g}$ for $30 \mathrm{~min}$ to pellet the fraction. The pellet was washed twice with ice-cold $\mathrm{H}_{2} \mathrm{O}$ (osmotic shock) and recovered by 10 min centrifugation at $12,000 \times \mathrm{g}$ to remove cytoplasmic and microsomal contaminants. To obtain pure myelin, the pellet was resuspended in $0.32 \mathrm{M}$ sucrose solution and loaded once again onto the $0.85 \mathrm{M}$ sucrose cushion. All subsequent steps were repeated a second time and the pure myelin pellet was resuspended in PBS supplemented with protease inhibitors. The isolation of myelin procedure is based on the W. Norton and S. Poduslo method [205], with modifications introduced in [206].

\section{Fluorescent labeling of myelin}

Myelin was purified and protein concentration was determined using BCA assay (see Section 2.2.1). For the fluorescent labeling of myelin with fluorescein isothiocyanate (FITC), a commercial kit for protein labeling was used (FITC Antibody labeling Kit, Thermo Scientific, Rockford, IL, USA). The reaction was performed in an aminefree borate buffer system, which allows the crosslinking reaction of FITC to primary amine groups of the amino acid lysine. For the labeling reaction $1 \mathrm{mg}$ of myelin was used and the labeling reaction was performed as recommended by the manufacturer.

\section{Myelin internalization assay}

For the myelin internalization assay, primary microglia were plated on coverslips and incubated for one to seven days in the appropriate growth media. Prior to 
the uptake assay, myelin was purified, labeled and diluted in growth medium. The uptake was performed by incubating cell cultures with myelin dilutions in a humid chamber for $2 \mathrm{~h}\left(37^{\circ} \mathrm{C}, 7 \% \mathrm{CO}_{2}\right)$. Subsequently, cells were fixed and processed for immunofluorescence analysis (see Section 2.1.2).

\subsubsection{Cytokine release assays}

For cytokine release assays, primary microglial cells were separated from the mixed glial culture by shaking and placed in 96 -well plates at densities of $1.5 \times 10^{5}$ cells per cavity [207]. After a 30 min attachment period, cells were extensively washed with DMEM containing 10\% FCS and kept in culture for one to three days. Cultures routinely consisted of more then $98 \%$ microglial cells as determined by staining with Griffonia simplicifolia isolectin B4 (Vector Laboratories, Burlingame, CA, USA). After one day, cells received either fresh medium (basal release values) or medium containing exosomes alone or in combination with $1 \mathrm{ng} / \mathrm{ml}$ LPS (E. coli R515, TLR ligand set 1 (APO-54N-018), Axxora/Appotech). Cells were incubated for $18 \mathrm{~h}$ and supernatants analysed. Cells were rinsed and given new medium with WST-1 agent for viability assay (Roche Diagnostics).

Measurements of cytokines and chemokines in culture supernatants were performed with factor- and species-specific complete ELISA systems or sets of capture and detecting antibody pairs as well as standard proteins (RD Systems, Biolegend) as described earlier [207]. The color reaction was measured at $450 \mathrm{~nm}$ on a microplate reader (Bio-Rad, Munich, Germany). Adaptations were made to reduce sample size and assay volume. Factors included TNF $\alpha$, IL 6, IL-10, IL-12p40 (covering the monomeric, homodimeric and heterodimeric versions, including IL-12p70 and IL-23), CXCL1 (KC, the mouse equivalent of GRO $\alpha$ ), CCL2 (MCP1), CCL3 (MIP1 $\alpha$ ), CCL5 (RANTES) and CCL22 (MDC).

The cytokine release assays was performed by Tommy Regen, Institute of Neuropathology, University of Göttingen, 37075 Göttingen, Germany. 


\subsubsection{Flow cytometric measurements}

Flow cytometric measurements were either performed with FACSCalibur (Becton

Dickinson, Germany) or FACSDiva (Becton Dickinson, Germany), respectively.

\section{Flow cytometric analysis of primary microglia}

For the flow cytometric measurements, primary microglia were placed into 12-well plate cavities at densities of 2.0 to $3.0 \times 10^{5}$ or into $10 \mathrm{~cm}$ dishes at a density of $1.0 \times 10^{6}$. After one day, the cells received either new medium, medium containing exosomes in various dilutions and/or IFN $\gamma$ (mouse recombinant, RD 485-MI/CF) at a final concentration of 10 to $100 \mathrm{ng} / \mathrm{ml}$. After $2 \mathrm{~h}$ to $2 \mathrm{~d}$ of incubation, cells were trypzinized and scraped into FACS Buffer (2\% FCS, 0.01\% EDTA, pH 8.0 in PBS). Subsequently, cells were processed for analysis by incubation with Fcreceptor blocking antibody followed by staining with directly fluorescent coupled antibodies (see Table 2.5. Cytometric data was analyzed with WinMDI software (Purdue University, USA) or FACSDiva software (Becton Dickinson, Germany) The flow cytometric measurements were partially performed by Denise van Rossum, Institute of Neuropathology, University of Göttingen, 37075 Göttingen, Germany.

Table 2.5: Directly-coupled antibodies (FACS)

\begin{tabular}{llll}
\hline Target & clone & Fluorophore/Specification & Reference \\
\hline CD11b & M1/70 & Alexa 488 & BD Pharmingen, BD Bioscience \\
CD16/CD32 & mouse BD Fc Block & - & BD Pharmingen, BD Bioscience \\
MHC class I & KH114v & Alexa 647 & BD Pharmingen, BD Bioscience \\
MHC class II & KH116 & Alexa 647 & BD Pharmingen, BD Bioscience
\end{tabular}

\section{Flow cytometric analysis of exosomes}

To analyze epitopes on the exosomal membrane, exosomes were purified and bound to the surface of beads (aldehyde/sulfate latex $4 \% \mathrm{w} / \mathrm{v}, 4 \mu \mathrm{m}$ Invitrogen) as described in [55]. Briefly, exosomes and beads were incubated overnight, washed and blocked with BSA in PBS. Subsequently, loaded beads were transferred to annexin V binding buffer and incubated with FITC-labelled annexin V (dilution 1:20) for 15 min. Afterwards, loaded beads were washed four times in annexin $\mathrm{V}$ binding buffer and 
acquired on a FACSCalibur (FACSCalibur, Becton Dickinson, Germany). The flow cytometric measurements were performed by Denise van Rossum, Institute of Neuropathology, University of Göttingen, 37075 Göttingen, Germany.

\subsubsection{T cell activation assay}

In co-culture experiments, indicated numbers $\left(5 \times 10^{4}, 1 \times 10^{5}\right.$ or $2 \times 10^{5} /$ well $)$ of primary microglia isolated from brains of C57BL/6 mice were plated in 96-well round bottom plates. Cells were stimulated overnight with $\operatorname{IFN} \gamma(10 \mathrm{ng} / \mathrm{ml})$, or left without stimulation, and then gently washed twice with DMEM containing 10\% FCS. Myelin oligodendrocyte glycoprotein MOG-specific $\mathrm{CD}^{+}{ }^{+} \mathrm{T}$ cells were isolated from TCR-transgenic 2D2 mice [208] by negative selection using magnetic beads (RD systems) as previously described [209]. $1 \times 10^{5}$ purified $\mathrm{CD} 4^{+} \mathrm{T}$ cells were mixed with microglial cells and recombinant MOG protein (rMOG), MOG peptide 35-55 (MOG35-55), recombinant ovalbumin, ovalbumin peptide 323-339 (ova323-339) or exosomes were added at indicated concentrations. Three days after stimulation, supernatants were collected and to determine IL-2 concentrations by ELISA. IL2 concentration was determined using a set of capture and detection antibodies against IL-2 (BD Biosciences) as well as purified standard cytokine. 2,2 $\alpha$-azino-bis3-ethylbenzthiazoline-6-sulphonic acid (ABTS; Sigma-Aldrich) was used as a substrate for detection and plates were read at $405 \mathrm{~nm}$. The T cell activation assay was performed by Gurumoorthy Krishnamoorthy, Department of Neuroimmunology, Max Planck Institute of Neurobiology, Martinsried, Germany.

\subsubsection{Stereotactic injection into the murine brain}

\section{Injection of IFN $\gamma$ into the hippocampus}

For the IFN $\gamma$ injection, eight to twelve weeks old NMRI mice were anethesized with a mix of ketamine/xylazin i.p and chucked into a custom made stereotactic frame. The skull was opened with a scalpel and the bregma was localized. The frame of the stereotact was adjusted to the bregma and the following coordinates were chosen to inject into the hippocampal area of the righten hemisphere (ap: $-0.24, \mathrm{ml}:-0.2$, 
dv: -0.2). A hole was drilled at the injection site into the cranium and the wound was cleaned with PBS. Afterwards, the needle was moved to the coordinates and lowered into the right depth. Subsequently, $1 \mu \mathrm{L} \operatorname{IFN} \gamma$ in PBS $(1 \mu \mathrm{g} / \mu \mathrm{L})$ or PBS were injected at a velocity of $250 \mathrm{~nL} / \mathrm{min}$. After injection, the needle was left for $5 \mathrm{~min}$ to allow distribution of the injected solution. The needle was removed and the wound was closed with Histoacryl ${ }^{\mathrm{TM}}$ (B. Braun Melsungen AG, Melsungen, Germany). Mice were observed for $24 \mathrm{~h}$ after injection, sacrificed and perfused.

The stereotactic injections were performed by Anja Schneider, Max Planck Institute for Experimental Medicine, 37075 Göttingen, Germany.

\section{Cryosectioning of mouse brains}

For cryosectioning, mice were perfused with $4 \%$ PFA in PBS, brains were prepared and washed in PBS. Brains were post-fixed for $2 \mathrm{~d}$ in $4 \% \mathrm{PFA}$ solution at $4^{\circ} \mathrm{C}$. Afterwards, brains were incubated in $20 \%$ sucrose solution. For sectioning, brains were cut into forebrain and cerebellum, mounted with Tissue-Tek ${ }^{\text {TM }}$ (Sakura, Torrance, CA, USA) on a metallic support and frozen in liquid nitrogen. The support was removed and the brain was positioned in the Leica CM1900 cryotom (Leica Microsystems, Mannheim, Germany). A section depth of $30 \mu \mathrm{m}$ was chosen and brains were cut. Finally, sections were transfered into PBS supplemented with $25 \%$ glycerol and $25 \%$ ethylenglycol and stored at $-20^{\circ} \mathrm{C}$.

\subsubsection{Immunohistochemistry}

Brain sections for immunohistochemistry were prepared as described in Section 2.2.11. For immunostainings, brain sections were washed three times in PBS, to remove glycerol and permeabilized in PBS with $0.5 \%$ Triton for 2 to 24 h. Afterwards, sections were incubated $1 \mathrm{~h}$ in $10 \%$ blocking solution (see Section 2.2.13), to reduce unspecific binding of antibodies (see below). Brain section were incubated with appropriate dilutions of primary antibodies (see Section 2.1.2) in 10\% blocking solution for $1 \mathrm{~h}(\mathrm{RT})$ or $16 \mathrm{~h}\left(4^{\circ} \mathrm{C}\right)$ respectively. After three washing steps of $10 \mathrm{~min}$ in PBS, the appropriate secondary antibodies were added (dilution 1:2000 in 10\% blocking solution) and sections were incubated for 1 to $4 \mathrm{~h}$ at RT. Excess antibody was re- 
moved by three washing steps in PBS at RT and sections were mounted onto glas slides. After drying of sections, Mowiol mounting medium (see below) was added and sections were covered with glas coverslips. The immunhistological stainings for MFGE8 were performed by Hannes Treiber, Max Planck Institute for Experimental Medicine, 37075 Göttingen, Germany.

\section{Blocking Solution (100\%)}

$2.0 \mathrm{~mL}$ Fetal Calf Serum

$2.0 \mathrm{~mL}$ Bovine Serum Albumine

$2.0 \mathrm{~g}$ Fishgelatine

filled up to $100 \mathrm{~mL}$ with PBS.

\section{MOWIOL (100 mL)}

2.4 g Mowiol (Calbiochem/Merck KGaA, Darmstadt, Germany)

$6 \mathrm{~g}$ Glycerol

$6 \mathrm{~mL} \quad \mathrm{H}_{2} \mathrm{O}$

were mixed and incubated at RT for $2 \mathrm{~h}$ with agitation. After addition of $12 \mathrm{~mL} \quad 0.2 \mathrm{M}$ Tris/HCl (pH 8.5),

the mixture was heated at $50^{\circ} \mathrm{C}$ for $10 \mathrm{~min}$.

The solution was cleared by centrifugation at $5000 \times \mathrm{g}$ for $15 \mathrm{~min}$ and $24 \mathrm{mg}$ DABCO reagent was added per $\mathrm{mL}$ of medium.

\subsubsection{Immunocytochemistry}

Immunocytochemistry was performed as described earlier in [210]. In brief, cells on coverslips were fixed for $10 \mathrm{~min}$ at RT with $4 \%$ PFA in PBS, followed by three washing steps in PBS. The cells were permeabilized with $0.1 \%$ Triton X-100 for 1 min at RT, then washed three times in PBS and $100 \%$ blocking solution was added to reduce unspecific binding of antibodies (Section 2.2.12). The cells were incubated with the primary antibodies in appropriate dilution in $10 \%$ blocking solution for $1 \mathrm{~h}$ at RT and washed three times for $5 \mathrm{~min}$ with PBS (see Section 2.1.2). Subsequently, cells were incubated with the secondary antibodies in required dilution in $10 \%$ blocking solution, washed three times in PBS and mounted in Mowiol mounting medium (see Section 2.2.12). 


\subsubsection{Microscopy and image processing}

\section{Two photon in vivo imaging of microglia}

For the in vivo analysis of exosome internalization, two-photon laser scanning fluorescence microscopy was applied. This technique developed in the late $20^{\text {th }}$ century permits imaging of tissues within the living animal. While imaging, two photons of comparably low energy excite one single fluorophore permitting the excitation of a very small focal volume. Thereby, two-photon microscopy combines the advantage of a focused excitation with a low background signal and low cytotoxicity [211].

In this study, in vivo experiments were carried out with CX3CR1/EGFP-transgenic mice [111] at ages between $70 \mathrm{~d}$ and $120 \mathrm{~d}$. General anesthesia was initiated by pentobarbital sodium i.p and continued with methohexital sodium (i.v.). Body temperature and electrocardiogram were monitored throughout the experiment. Spinal cord segments L4 and L5 were exposed by laminectomy of L1 and L2 spines for imaging of the dorsal white matter. To avoid movement caused by active respiration, the mice were paralyzed with pancuronium bromide. The spinal column was rigidly fixed by custom-made clamps and the dura mater was carefully removed. A region of interest was selected and a microcapillary positioned. With a delay of 30 to 60 minutes, PKH26-labelled exosomes derived from $1.5 \times 10^{5}$ Oli-neu cells were injected into the respective area. In vivo-imaging was performed by a custom-made 2P-LSM equipped with a fs-pulsed titanium-sapphire laser (Chameleon Ultra II; Coherent, Glasgow, UK) and a Zeiss $20 \times / 1.0$ water immersion objective. Uniformly spaced $(2 \mu \mathrm{m})$ planes of $250 \times 250$ to $500 \times 500 \mu \mathrm{m}$ regions of the spinal dorsal columns were recorded and processed to obtain z-stacks of images $(512 \times 512$ pixels in size $)$. Image processing was performed using Matlab (version 7, MathWorks, Ismaning, Germany) and Image ${ }^{3}$. The in vivo imaging of microglia was performed by Payam Dibaj, Department of Neurology, University of Göttingen, 37075 Göttingen, Germany.

\footnotetext{
${ }^{3}$ http://rsbweb.nih.gov/ij
} 


\section{Confocal microscopy}

For the subcellular localization of exosomes in microglia, laser scanning confocal microscopy was used, which was originally developed by M. Minsky in the middle of the $20^{\text {th }}$ century [212]. Due to the laser light excitation and pinhole-based rejection of the off-focus light, confocal microscopy provides higher resolution images with less background than conventional light microscopy [213]. Confocal microscopic images were acquired with Leica DMIRE2 microscope and a Leica TCS SP2 AOBS confocal laser scanning setup (Leica Microsystems, Mannheim, Germany) or with a Zeiss LSM 510 confocal microscope (Zeiss, Jena, Germany). 40×, NA 1.25 or $63 \times$, NA 1.4 oil plan-apochromat objectives (Leica Microsystems, Mannheim, Germany) or $40 \times$ or a $63 \times$ oil-immersed objective (Zeiss, Jena, Germany) were used for image acquisition. For live cell imaging, temperature control was applied to the microscope setup (see Section 2.2.5). Images were processed and analyzed with Image ${ }^{4}$. To estimate colocalization of two proteins by immunocytochemistry, both proteins were detected with antibodies coupled to distinguishable fluorophores. Each color channel was imaged separately and colocalization was determined using the colocalization module of Meta Imaging Series 6.1 software (Universal Imaging Corporation, West Chester, PA, USA).

\section{Electron microscopy}

For electron microscopy analysis, exosomes were purified, fixed with $2 \%$ paraformaldehyde and adsorbed to glow-discharged formvar-carbon-coated nickel grids. The samples were post-fixed for 2 min with $1 \%$ glutaraldehyde and stained with annexin- $\mathrm{V}$ (Sigma-Aldrich) and immune-gold-labelled. Observations were made with an electron microscope (Leo EM912AB; Zeiss), and images were aquired using an on-axis $2048 \times 2048$ charge-coupled device camera (Proscan; Schering). The Electron Microscopy was performed by Wiebke Möbius and Giselheid Schulz, Max Planck Institute for Experimental Medicine, 37075 Göttingen, Germany.

\footnotetext{
${ }^{4}$ http://rsb.info.nih.gov/ij/
} 


\section{Chapter 3}

\section{Results}

\subsection{Exosomes are released by oligodendrocytes}

In this study we focused on the functional analysis of oligodendroglial exosomes, that had been previously identified by us and other groups [4-6]. Besides exosomal proteins common to exosomes derived from different cell types, exosomes contain proteins highly specific for their parenting cell [33, 44, 45]. Therefore, we started the project by analyzing the composition of exosomes released by the oligodendroglial cell line, Oli-neu, and by primary oligodendrocytes of new born rats in vitro.

\subsubsection{Isolation and characterization of oligodendroglial exosomes}

For the isolation of oligodendroglial exosomes we used a protocol combining a series of centrifugation steps with increasing centrifugal forces. After collecting exosomes in the respective cell culture medium, several centrifugation steps were applied to remove cell debris, followed by final pelleting of vesicles at $100,000 \times \mathrm{g}$. This final pellet has been previously shown to contain small vesicles of 50 to $100 \mathrm{~nm}$ in size, resembling the vesicle diameter of exosomes [5]. In addition, Western blot analysis of the $100,000 \times \mathrm{g}$ pellet, isolated from rat primary oligodendrocytes, revealed the abundance of exosomal marker proteins flotillin-2 and alix. Furthermore, major myelin proteins proteo-lipid protein (PLP) and myelin oligodendrocyte glycoprotein (MOG) were also detected, whereas calnexin as a marker protein for vesicles derived 


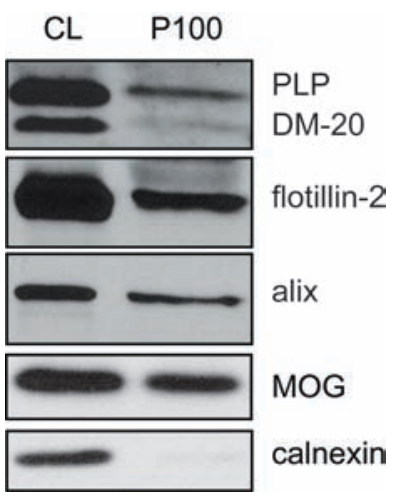

Figure 3.1: Exosomes of rat primary oligodendrocytes contain exosomal marker and major myelin proteins.

Exosomes were isolated from the culture medium of mature rat oligodendrocytes primary cultures by sequential centrifugation steps. The cell lysate ( $C L ; 1 / 20$ of the total lysate) and the exosome fraction $100,00 \times \mathrm{g}$ pellet (P100) were analyzed by Western blotting for the presence of myelin oligodendrocyte glycoprotein (MOG), proteo-lipid protein (PLP), flotillin-2, alix and calnexin.

from the endoplasmatic reticulum was absent from the $100,000 \times \mathrm{g}$ pellet (see Figure 3.1).

Sucrose gradient analysis of the $100,000 \times \mathrm{g}$ pellet

To verify the accuracy of our purification method, we also analyzed the $100,000 \times \mathrm{g}$ pellet collected from the mouse oligodendroglial cell line Oli-neu on a continuous sucrose gradient. In addition to the sequential centrifugation, this method permits the separation of vesicles according to their density [13] (see Figure 3.2). Western blot analysis illustrated, that fractions corresponding to the appropriate density of exosomes (1.15 to $1.19 \mathrm{~g} / \mathrm{mL}$ sucrose) contained exosomal marker proteins alix, Tsg101 and flotillin-2. Moreover, fractions of exosomal density derived from transiently transfected Oli-neu cells additionally contained myelin proteins PLP and MOG. The lack of calnexin signals in fractions of 1.18 to $1.25 \mathrm{~g} / \mathrm{mL}$ indicated that 


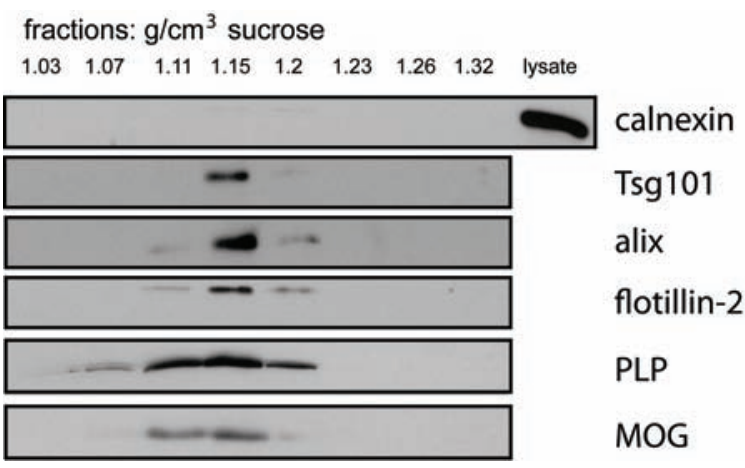

Figure 3.2: Exosomes of the oligodendroglial cell line Oli-neu contain typical exosomal marker proteins.

Exosomes were purified from the culture medium of the oligodendroglial precursor cell line Oli-neu by sequential centrifugation steps with increasing centrifugal forces up to $100,000 \times \mathrm{g}$. The $100,000 \times \mathrm{g}$ pellet was loaded onto continuous sucrose density gradients and centrifuged for $16 \mathrm{~h}$. Fractions of equal volume were collected and further centrifuged at $100,000 \times \mathrm{g}$. The pellets thus obtained were subjected to Western blot analysis. After transient transfection of the cells, PLP and MOG are recovered from fractions with the characteristic density of exosomes that contain the exosomal marker proteins, alix, Tsg101 and flotillin-2.

we were able to exclude ER-derived vesicles from the exosome preparation [13].

\subsubsection{Quantitative analysis of oligodendroglial exosome release}

To follow the fate of exosomes once they leave the parenting cell, we were interested in how much material leaves the cell through the exosomal pathway. To address this question we pulsed Oli-neu cells and primary oligodendrocytes with $\mathrm{S}^{35}$-Met/-Cys for $1 \mathrm{~h}$ and followed the release of labeled proteins via exosomes in comparison to cellular degradation for a time span of $24 \mathrm{~h}$ (Figure 3.3). While the majority of cellular proteins were degraded within the cell, 3.6\% (Oli-neu cells) to $4.7 \%$ (primary oligodendrocytes) of proteins synthesized during $1 \mathrm{~h}$ of radioactive pulse were released within $24 \mathrm{~h}$ via the exosomal pathway. 

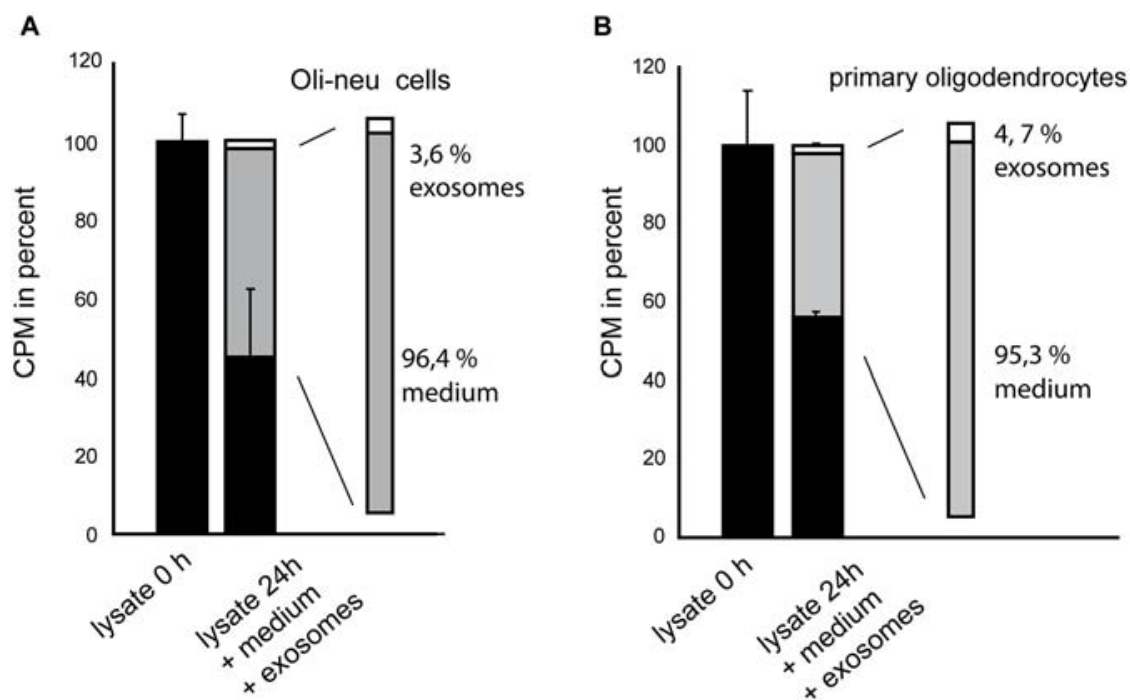

Figure 3.3: Quantitative analysis of exosome release by oligodendrocytes.

Oli-neu cells (A) or primary oligodendrocytes (B) were pulsed with $S^{35}$-Met/-Cys for $1 \mathrm{~h}$ and cells were chased for $24 \mathrm{~h}$. Cells lysates, medium and exosomes were collected at time points $0 \mathrm{~h}$ and $24 \mathrm{~h}$, respectively and radioactive decay was measured. Up to $4.7 \%$ of cellular proteins synthesized during $1 \mathrm{~h}$ of pulse were found in the exosomal fraction after $24 \mathrm{~h}$.

\subsection{Exosomes are specifically internalized by microglia}

\subsubsection{Internalization of oligodendrocyte-derived exosomes by mi- croglia}

To follow the fate of exosomes, purified Oli-neu cell line exosomes were labeled with the lipophilic dye PKH67 and incubated with primary cultures of mouse oligodendrocytes, astrocytes, cortical neurons and microglia. After $2 \mathrm{~h}$ incubation at $37^{\circ} \mathrm{C}$, cells were fixed and analyzed by confocal microscopy. The results showed that exosomes were mainly internalized by microglia, while almost no exosomes were found inside or attached to the surface of oligodendrocytes, astrocytes or neurons (Figure 3.4). Furthermore, confocal analysis revealed that exosomes were not attached to the plasma membrane, but were indeed internalized by microglia. We found that exosome co-localize with Lamp-1, a resident protein of the late endosomes/lysosome, 

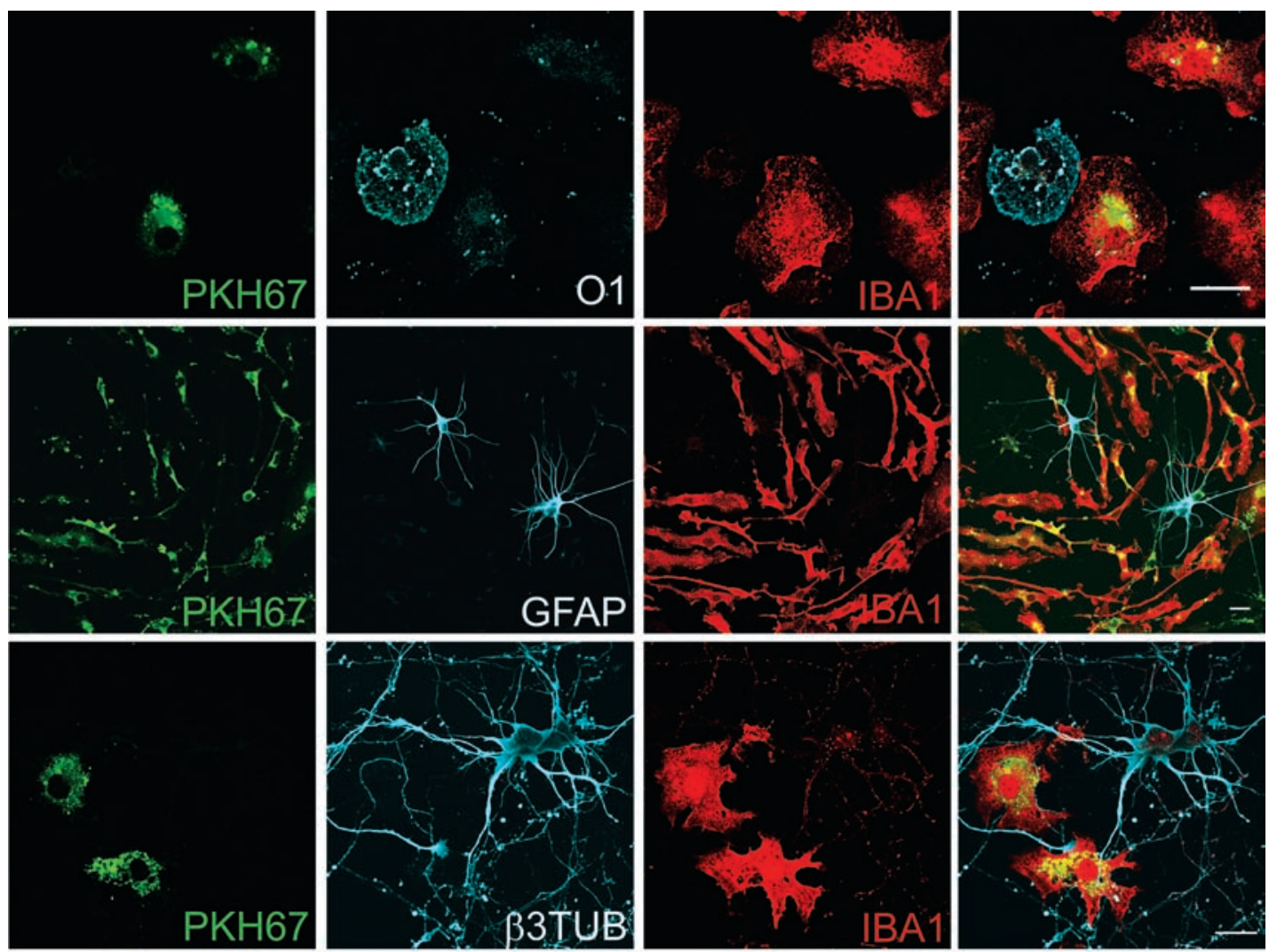

Figure 3.4: Oligodendroglial exosomes are selectively internalized by microglia. Purified exosomes $(100,000 \times$ g pellet) were labeled with the dye PKH67 and added to primary cultures of mouse oligodendrocytes, astrocytes, cortical neurons and microglia. After incubation with exosomes for $2 \mathrm{~h}$ at $37^{\circ} \mathrm{C}$, cells were fixed and analyzed by confocal microscopy. Oligodendrocytes, astrocytes, neurons and microglia were recognized by staining with antibodies against GalC (O1), GFAP, $\beta 3$-tubulin and Iba1, respectively. Exosomes were taken up by Iba1-positive microglia. Scale bars, $20 \mu \mathrm{m}$.

confirming that exosomes are internalized and transported to functional subcompartments within the cell (Figure 3.5). Similar results were obtained with exosomes isolated from rat primary oligodendrocytes (see Figure 3.6).

To exclude the passive diffusion of PKH67 dye into the plasma membrane of microglia, we confirmed our previous findings using multiple approaches. To rule out diffusion, we labeled the $100,000 \times \mathrm{g}$ pellet with PKH67 and incubated the exo- 

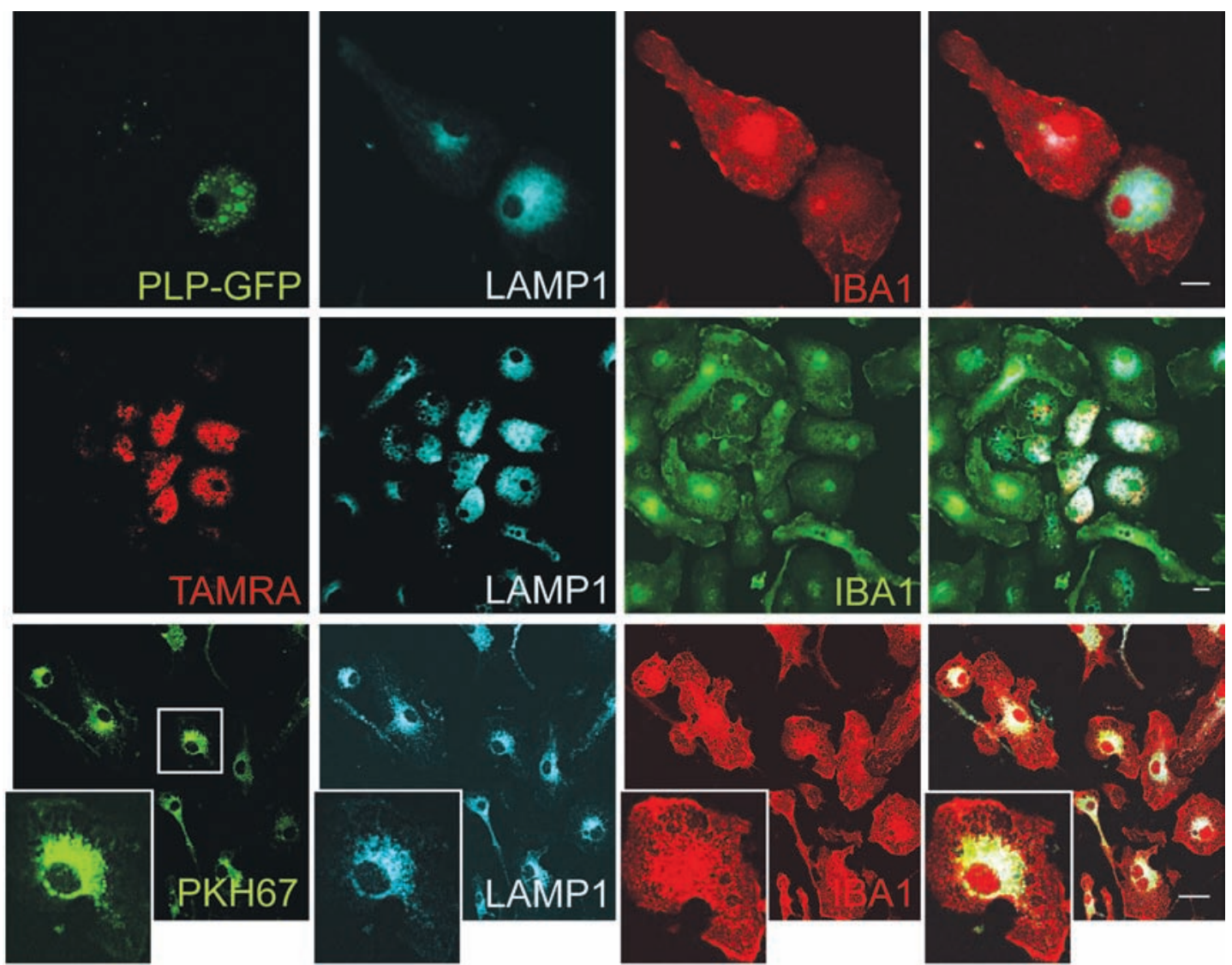

Figure 3.5: Oligodendroglial-derived exosomes are transferred to late endosomes/ lysosomes. Exosomes were purified from Oli-neu cells expressing PLP-GFP or from wild-type cells and subsequently labeled with the lipophilic dye PKH67 or through the crosslinking of carboxytetramethylrhodamine (TAMRA) to proteins of the exosomal membrane fraction. PLP-GFP positive or fluorescent labeled exosomes were added to primary microglia cultures and incubated for $2 \mathrm{~h}$ at $37^{\circ} \mathrm{C}$. For microscopic analysis, microglia were visualized using lba1 antibody. Internalized exosomes colocalize with Lamp1 , a marker for late endosomes/lysosomes in primary microglia. Scale bar, $10 \mu \mathrm{m}$.

some suspension with living or PFA-fixed primary microglia cultures. After $1 \mathrm{~h}$ of incubation we detected internalization of exosomes into the living cells. However, we did not find any PKH67 staining on PFA-fixed microglia (see Figure 3.7). Furthermore, we used alternatively labeled exosomes. In this approach, the $100,000 \times \mathrm{g}$ pellet was labeled by cross-linking of carboxytetramethyl-rhodamine (TAMRA) to 

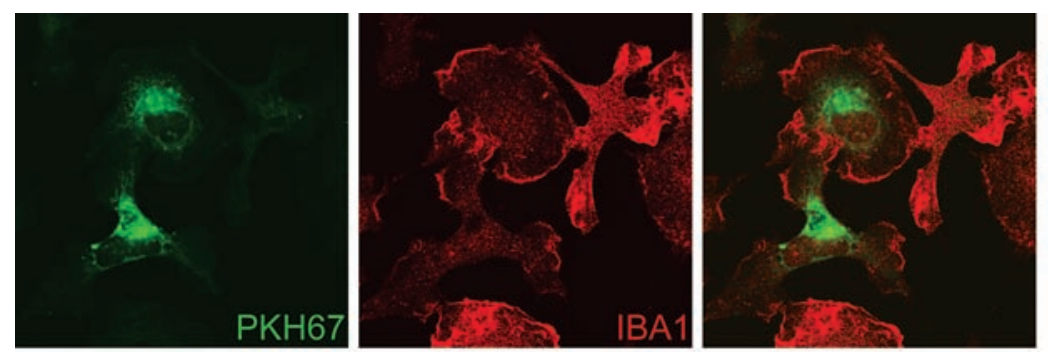

Figure 3.6: Exosomes of rat primary oligodendrocytes are internalized into primary microglia. Exosomes from primary cultures of mature rat oligodendrocytes were purified, labeled with PKH67 (green) and incubated with microglia labeled with Iba1 (red) at $37^{\circ} \mathrm{C}$ for $1 \mathrm{~h}$. Scale bar, $10 \mu \mathrm{m}$

the exosomal proteins. After removal of unbound dye, the exosomes were incubated with primary microglia. Again, we found that exosomes were internalized and transported to the late endosomal/ lysosomal compartment of the cell (see Figure 3.5). Similar results were obtained with PLP-GFP positive exosomes purified from transiently transfected Oli-neu cells (see Figure 3.5). Finally, we labeled the 100,000 $\times \mathrm{g}$ pellet with PKH67 and further purified the comprised vesicles on a sucrose gradient. All the fractions covering a density from 1.18 to $1.25 \mathrm{~g} / \mathrm{mL}$ were recovered, diluted and enclosed vesicles were collected by ultracentrifugation. After pelleting, the fractions were used for internalization assays. Microscopic analysis revealed that the majority of internalization was found from fractions with the characteristic exosomal density, thereby excluding non-specific transfer of dye to microglia due to aggregation or differently sized vesicles (see Figure 3.8). 


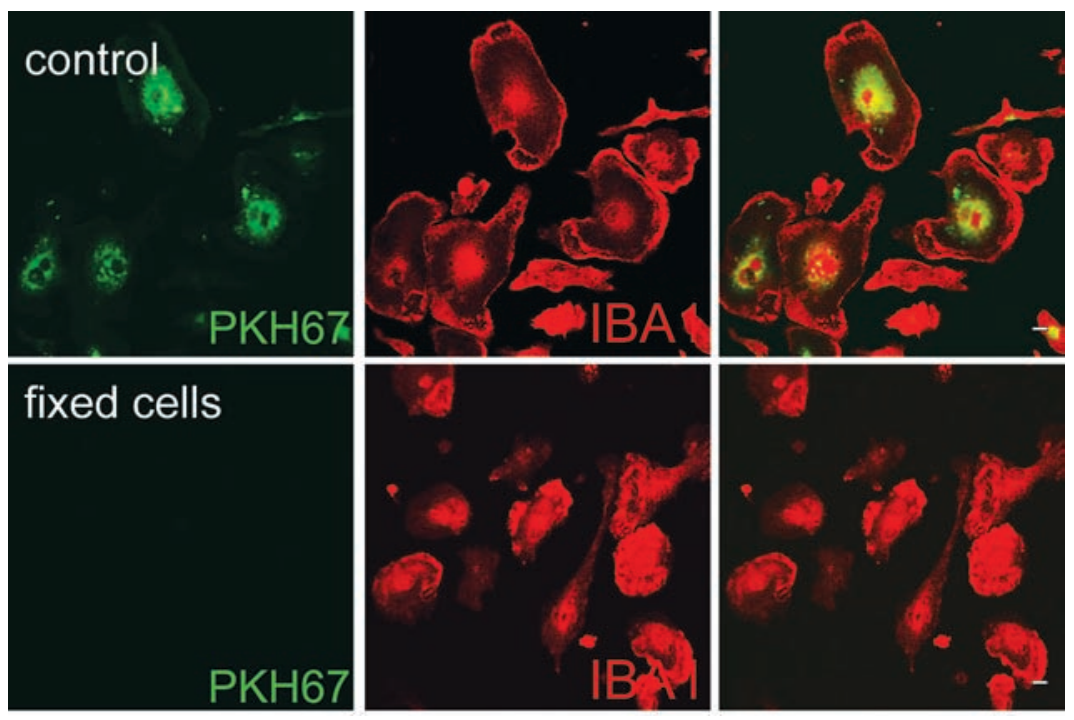

Figure 3.7: The lipophilic dye PKH67 does not diffuse into the plasma membrane of microglia. Exosomes were purified, labeled with PKH67 (green) and incubated either with living or with PFA fixed primary microglia cultures (lba1, red) for $1 \mathrm{~h}$ at $37^{\circ} \mathrm{C}$. PKH67labeled exosomes do not transfer the dye to fixed cells. Scale bar, $10 \mu \mathrm{m}$. 

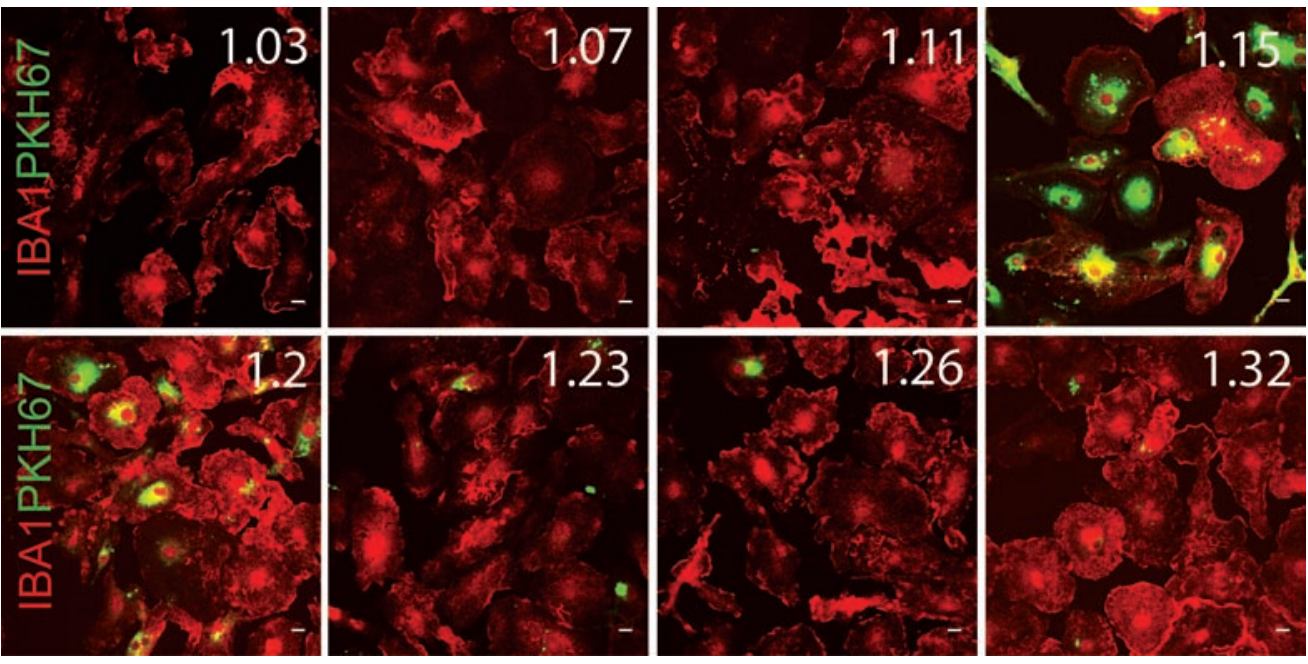

Figure 3.8: Internalization pattern of sucrose-gradient purified vesicles in microglia is identical to exosomes. Culture medium of Oli-neu cells were subjected to sequential centrifugation steps with increasing centrifugal forces up to $100,000 \times \mathrm{g}$. The $100,000 \times \mathrm{g}$ pellet was labeled with $\mathrm{PKH} 67$ and loaded onto a sucrose gradient (densities 1.03 to $1.32 \mathrm{~g} / \mathrm{cm}^{3}$ ). After $16 \mathrm{~h}$ of centrifugation, fractions were collected, comprised vesicles pelleted at $100,000 \times \mathrm{g}$ and resuspended in $100 \mu \mathrm{L}$ PBS. $60 \mu \mathrm{L}$ of each fraction was added to primary microglial cultures and incubation continued for $2 \mathrm{~h}$ at $37^{\circ} \mathrm{C}$. Cells were visualized for PKH67 (green) and lba1 (red). The densities of the sucrose gradient fractions are indicated. The majority of uptake occurred from fractions with the characteristic density of exosomes. Scale bar, $10 \mu \mathrm{m}$. 
A
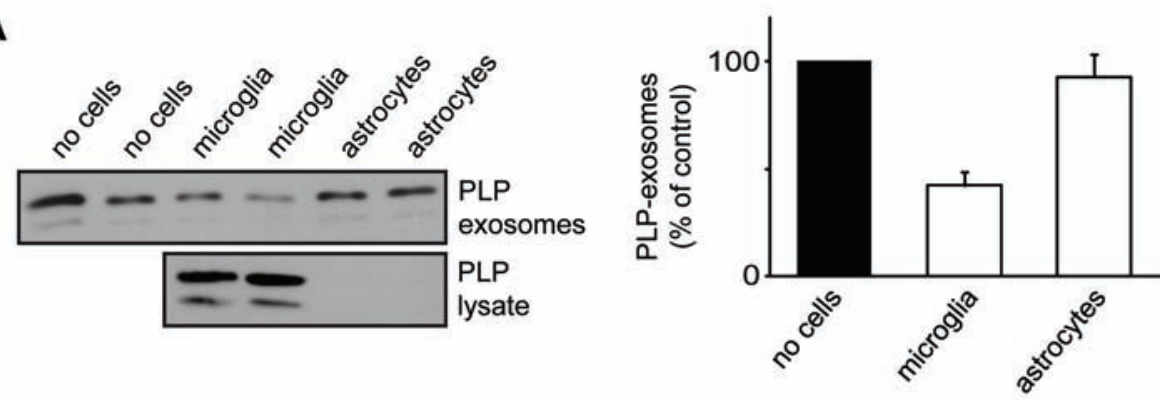

B

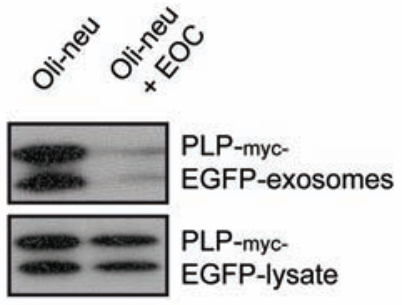

C
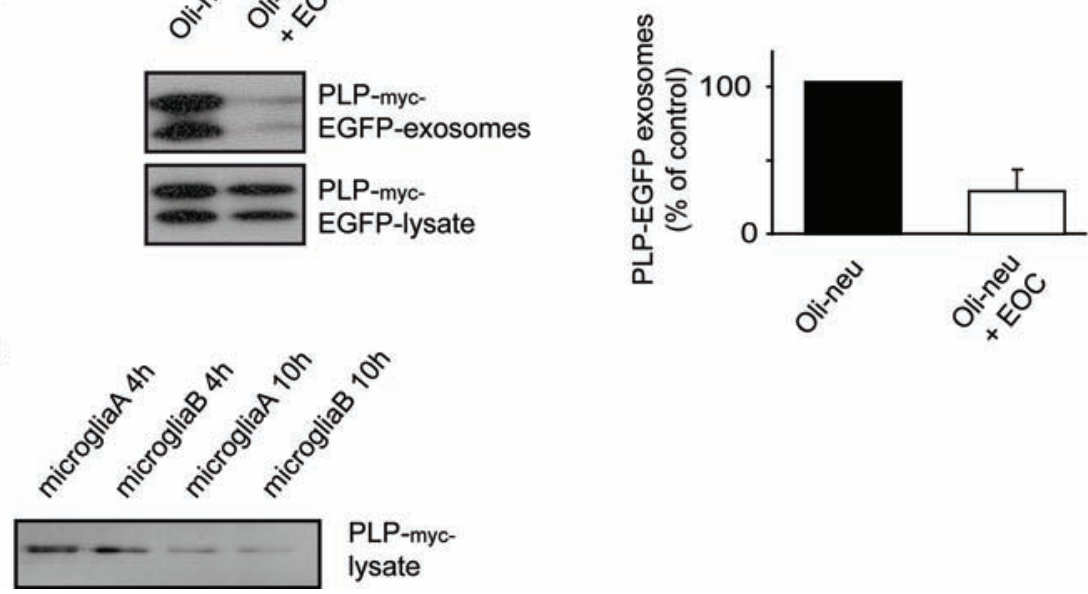

Figure 3.9: Exosomes are specifically cleared by microglia but not by astrocytes.

(A) The oligodendroglial cell line Oli-neu stably expressing PLP-myc-EGFP was (co) cultured with or without the microglial cell line EOC-20 for $24 \mathrm{~h}$. Exosomes were subsequently isolated from the culture medium and the amount of PLP-myc-EGFP determined by Western blotting with primary antibodies against the myc epitope. Equal amounts of proteins $(20 \mu \mathrm{g})$ from the cell lysates were loaded onto the gel. In the presence of microglia, a significant reduction of PLP-myc-EGFP in the culture medium was observed. Values are given as mean $+/$ - SD $(n=3)$. (B) Culture medium of mature primary oligodendrocyte cultures was added to dishes without cells (no cells) or to an equal number of primary microglia or astrocytes (two independent experiments as shown). After $8 \mathrm{~h}$ of incubation, exosomes were purified from the culture medium and the amount of PLP was determined. After co-culture with primary microglia, less PLP was found in the medium and more PLP accumulated in the microglial cell lysate, as compared to cultures with primary astrocytes. Only a fraction (1/5) of the total culture medium was loaded on to the gel. Values are given as the mean $+/-S D ; n=3$. (C) To analyze whether exosomal PLP-myc is degraded after its uptake in microglia, we added purified exosomes to primary microglia, allowed uptake to occur for $4 \mathrm{~h}$ and harvested the cells after 4 and $10 \mathrm{~h}$. PLP-myc signals on the Western blot were detected using anti-myc antibodies. PLP decreases with time in the microglial cell lysate. Two independent experiments are shown. 
We verified our microscopic findings in continuative biochemical experiments. Oligodendroglial and microglial cell lines were cultured together for $24 \mathrm{~h}$, and then the amount of exosomes in the cell culture medium was determined. In co-cultures of Oli-neu cells stably expressing PLP and the microglial cell line EOC-20, we observed that PLP-positive exosomes were efficiently cleared from the cell culture medium (see Figure 3.9, A). In similar experiments with primary cells, we collected exosomes in the supernatant of oligodendrocyte cultures, cleared cell debris from the medium by centrifugation (once at $3,000 \times \mathrm{g}$ and twice at $4,000 \times \mathrm{g}$ ) and incubated the medium with primary cultures of microglia and astrocytes. After $8 \mathrm{~h}$, we collected the medium, purified exosomes and accessed the amount of exosomal PLP (see Figure $3.9, \mathrm{~B})$. We could show that in the presence of microglia, PLP-positive exosomes were cleared from the medium and that oligodendroglial PLP is additionally found in the cell lysate of microglia. In a striking contrast, we did not find any differences in the amount of exosomes in the medium after incubation with astrocytes nor did we find PLP in the respective cell lysate. Interestingly, an estimation of the exosomal uptake efficiency by microglia revealed, that $3 \times 10^{5}$ primary microglia were capable of clearing $40 \%$ of PLP-positive exosomes produced by $1.5 \times 10^{6}$ primary oligodendrocytes within $8 \mathrm{~h}$.

Finally, we determined whether exosomal PLP is degraded by micoglia. Therefore, we added purified exosomes to primary microglia cultures, allowed internalization to occur and harvested the cells at different time points. The results showed that PLP is continuously degraded by microglia over time (see Figure 3.9, C). 


\subsubsection{Exosomes are internalized by microglia in vivo}

Taken together, the in vitro data clearly illustrated, that oligodendroglial membrane components are transfered by exosomes to microglia. In the next step, we wanted to affirm these findings by showing the internalization of exosomes by microglia in vivo. For these experiments we used transgenic mice with insertion of the enhanced green fluorescent protein (eGFP) into the Cx3cr1 locus, resulting in a specific expression of eGFP in the resident microglia of the CNS [214]. Exosomes were labeled and injected into the spinal cord. After $30 \mathrm{~min}$, images were acquired by two-photon microscopy for a time span of $60 \mathrm{~min}$. The time lapse imaging series shown in Figure 3.10 (A) revealed that exosomes were indeed internalized by eGFP-positive microglia and transported along cellular processes towards the cell body. In addition, exosomes were found in Lamp-1 positive organelles of the microglia (see Figure 3.10, B), as it was also observed in cell culture experiments in vitro. In total, approximately $50 \%$ of the eGFP-postive microglia at an approximate distance of $400 \mu \mathrm{m}$ from the injection site showed co-localization with exosomes, thereby confirming that microglia possess exosome clearing activity. 
A
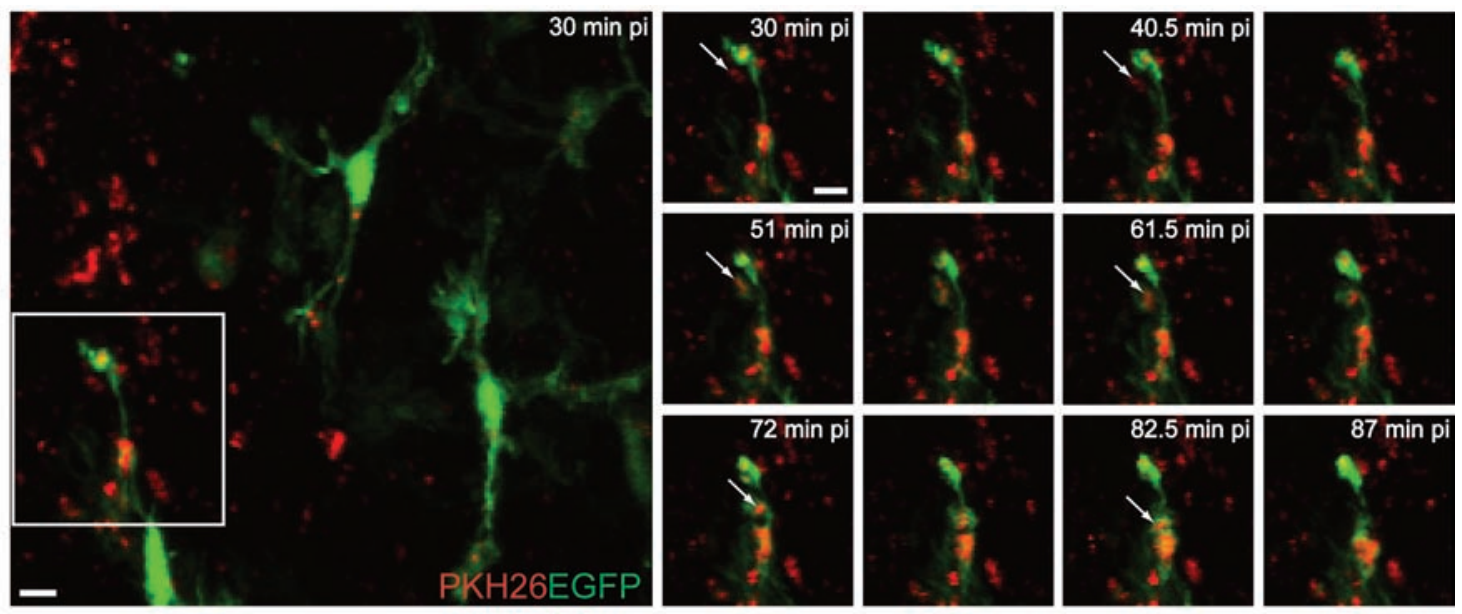

B
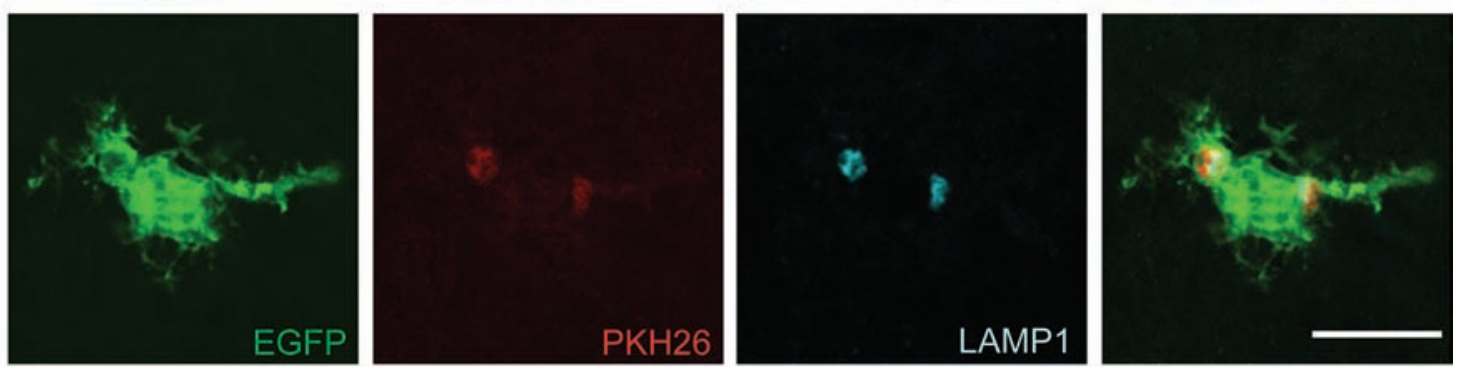

Figure 3.10: Exosomes are internalized by microglia in vivo. (A) Purified oligodendroglial exosomes were labeled with the dye PKH26 (red) and injected into the spinal cord of anesthetized CX3CR1/GFP mice. Time lapse imaging was performed by twophoton microscopy. The boxed area in the left image is shown as a time lapse series. Fluorescently labeled exosomes moving into and within microglia are indicated by arrows at different time points. The elapsed time after injection of exosomes (post-injection, pi) is shown in the images. Scale bars, $10 \mu \mathrm{m}$. (B) Immunohistochemistry of spinal cord sections of CX3CR1/GFP mice injected with PKH26-labeled exosomes (red) two hours before perfusion. Confocal microscopy images of microglia expressing EGFP and internalized exosomes. Sections were stained for LAMP-1 (cyan). Scale bar, $10 \mu \mathrm{m}$. 


\subsection{Microglia internalize exosomes by macropinocyto- sis}

\subsubsection{Which endocytic pathway conveys exosome internalization by microglia?}

Microglia, as well as other mammalian cell types, exhibit various endocytic pathways. Among them are phagocytosis, exclusively found in macrophages, and a group of pinocytotic mechanisms, including clathrin- or caveolin-mediated endocytosis and macropinocytosis [175]. The coordinated action of deforming the plasma membrane and the scission of these intermediates from the plasma membrane requires an interplay of proteins, which are diverse and characteristic for each of the distinct endocytic pathways. To define the entry pathway of exosomes into microglia, we treated the cells with various pharmacological inhibitors. All inhibitors were directed against proteins involved in the endocytic pathways. In these extensive studies, we tested the influence of dynasore, inhibiting the GTPase activity of dynamin [215] and NSC23766, a small molecule inhibitor of Rac1-GTPase [216]. Furthermore, we analyzed the influence of cytochalasinD, which interferes with the polymerization of the actin cytoskeleton [217]. Moreover, we tested amiloride, which blocks the $\mathrm{Na}^{+} / \mathrm{H}^{+}$exchanger of the lysosomal compartment [218]. After performing exosome internalization assays in presence of above mentioned drugs, we observed a clear reduction in the exosomal uptake (see Figure 3.12). These findings are consistent with a macropinocytotic mechanism. Since, functional acidification of the lysosomal compartment is crucial for macropinocytosis, we treated microglia further with alkalizing drugs bafilomycin A, monensin and chloroquine [219, 220]. Treatment with each of these drugs resulted in a significant inhibition of exosome internalization (see Figure 3.12, B). During macropinocytosis, different modes of macropinosome formation have been described [221]. Both lamellipodia-like ruffles and blebbing are known to be the initial steps of macropinocytosis. We tested the latter, by inhibiting of blebbing through blebbistatin, an inhibitor of myosinII-dependent blebbing [221], but did not observe any influence on exosome internalization (Figure 3.12). 

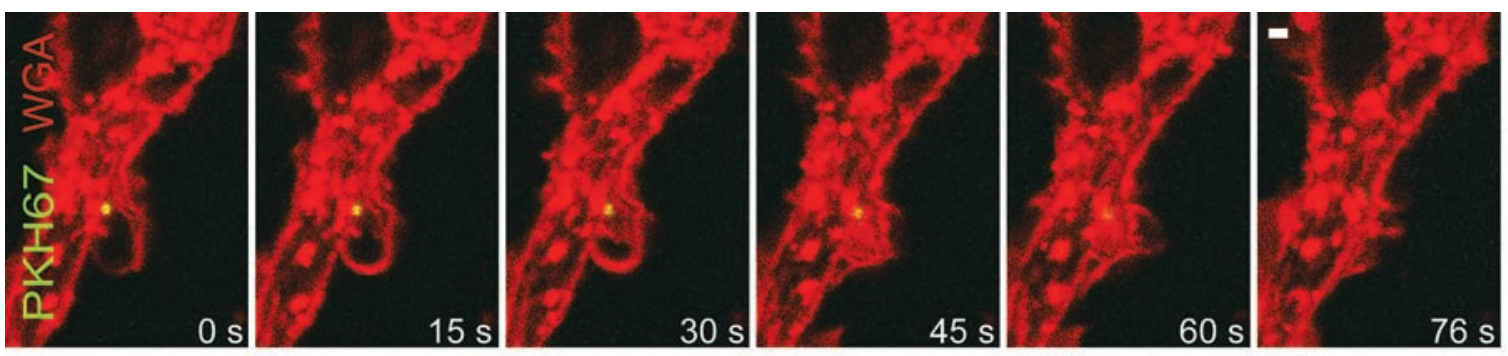

Figure 3.11: Macropinocytosis of exosomes, visualized by live cell imaging of microglial EOC cells. Purified exosomes labeled with PKH67 (green) were incubated with EOC-20 cells labeled with WGA (red) and examined by confocal live microscopy at $37^{\circ} \mathrm{C}$. An image sequence spanning $76 \mathrm{~s}$ showing engulfment of exosomes by large lamellipodia-like ruffles is displayed. Scale bar, $1 \mu \mathrm{m}$.

Furthermore, we performed live imaging of exosome internalization (see Figure 3.11). We incubated purified exosomes with the microglial cell line EOC-20 and observed exosome internalization with initial formation of membrane protrusions. Exosomes were observed to be enclosed by large lamellipodia-like ruffles and were finally engulfed by the cell. Consistent with the lack of inhibition of exosome internalization by blebbistatin, we did not observe blebbing on the plasma membrane of the microglia. 
A

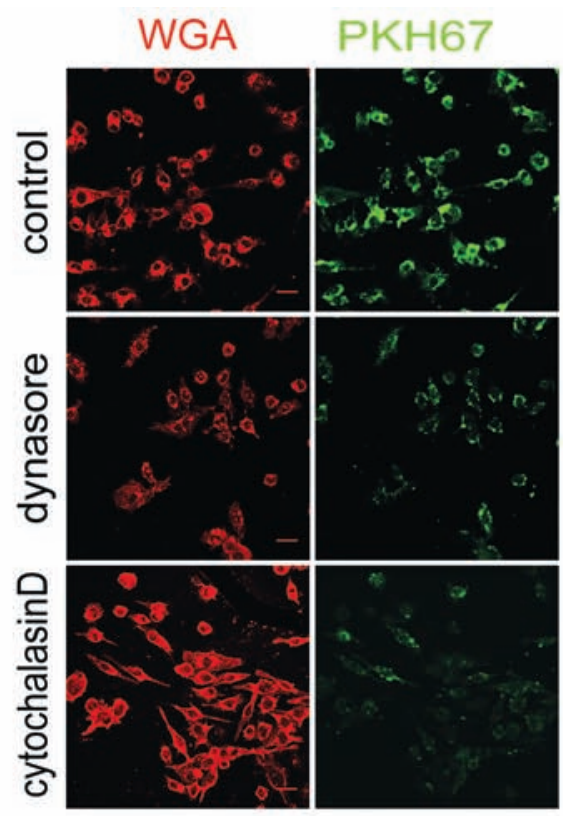

B

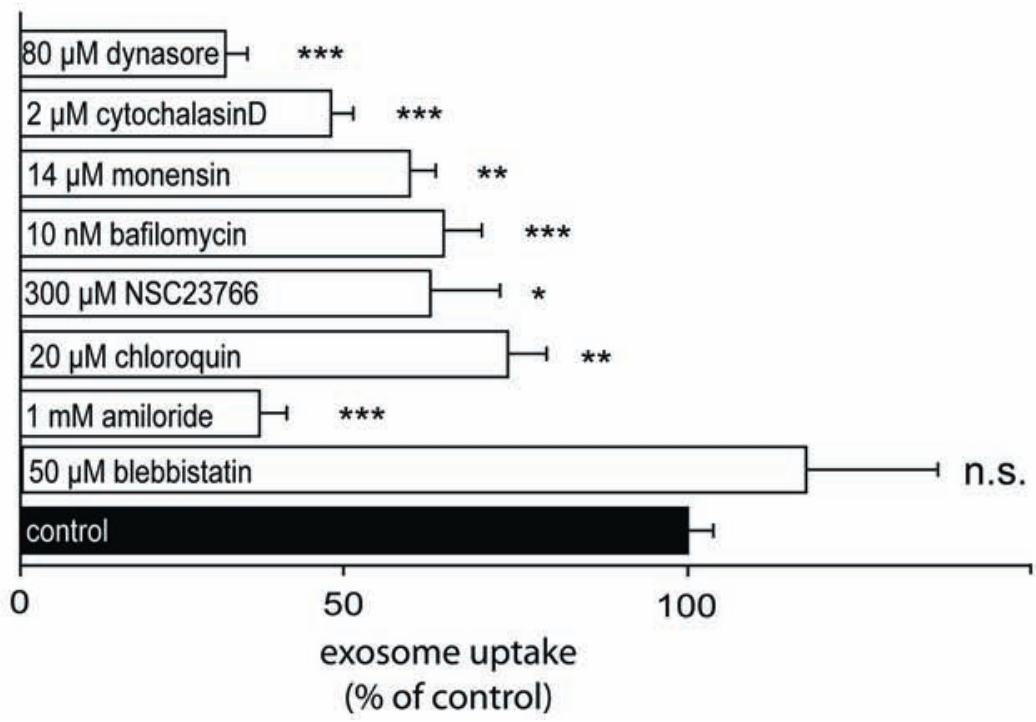

Figure 3.12: Microglia internalize exosomes via macropinocytosis. Purified exosomes from the oligodendroglial cell line Oli-neu were labeled with the dye PKH67 (green) and incubated for $2 \mathrm{~h}$ at $37^{\circ} \mathrm{C}$ together with the microglial cell line EOC-20. Cells were incubated with the indicated inhibitors (dynasore, chloroquin, monensin, bafilomycin, NSC23766, cytochalasinD, amiloride, blebbistatin) for $30 \mathrm{~min}$ prior and during the $2 \mathrm{~h}$ incubation. Cells were subsequently fixed, stained with rhodamine-conjugated wheat germ agglutinin (WGA) (red) and uptake was determined by confocal microscopy and image analysis. Changes in exosome internalization were quantified from three independent experiments. Values represent means ${ }^{+} /$- SEM (n.s., not significant; ${ }^{*} p<0.05$; ${ }^{* *} p<0.01$; $\left.{ }^{* * *} p<0.001\right)$. 


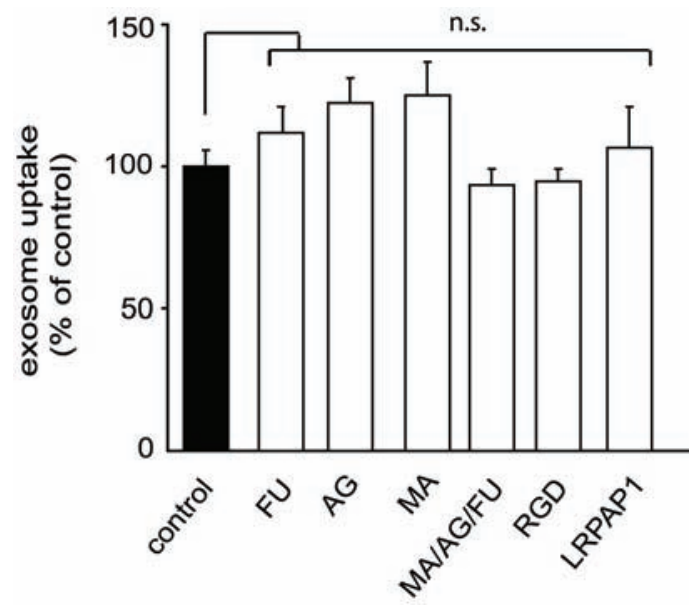

Figure 3.13: Microglia internalize exosomes independently of phagocytic receptors. Purified exosomes were incubated with EOC-20 cells together with the indicated substances for $30 \mathrm{~min}$ before and during the two hours of incubation. Exosome uptake was determined by microscopy and image analysis. Treatment with fucoidan (FU), Nacetyl-D-glucosamine (AG), D-mannosamine-hydrochloride (MA), a combination of AG, MA and FU, the peptide -GRGDSP- (RGD), and the LDL-receptor-related protein associated protein 1 (LRPAP1) did not influence the internalization of exosomes. Values are given as means +/ - SEM ( $n=3$; n.s., non significant.)

\subsubsection{Is the internalization of exosomes receptor dependent?}

To study the involvement of receptors during internalization of exosomes, we used specific inhibitors of known microglial cell surface receptors (see Figure 3.13). Fucoidan inhibits both scavanger receptor class A and B interactions [222], but did not influence the exosome internalization by microglia. Since lectins have been described as receptors in phagocytosis [223], we tested specific antagonists mannosamine and $\mathrm{N}$-acetylglucosamine alone or in combination with Fucoidan. None of these compounds affected the internalization, either alone or in combination. Likewise, incubation of microglia with RAP/LRPAP1 that is blocking the uptake via the low-density lipoprotein receptor revealed no influence. Moreover, the RGDpeptide antagonizing the integrin-mediated internalization also had no effect (see Figure 3.13). Consistent with these findings, we did not detect a significant binding of exosomes to the surface of microglia at low temperature (see Figure 3.14). The 


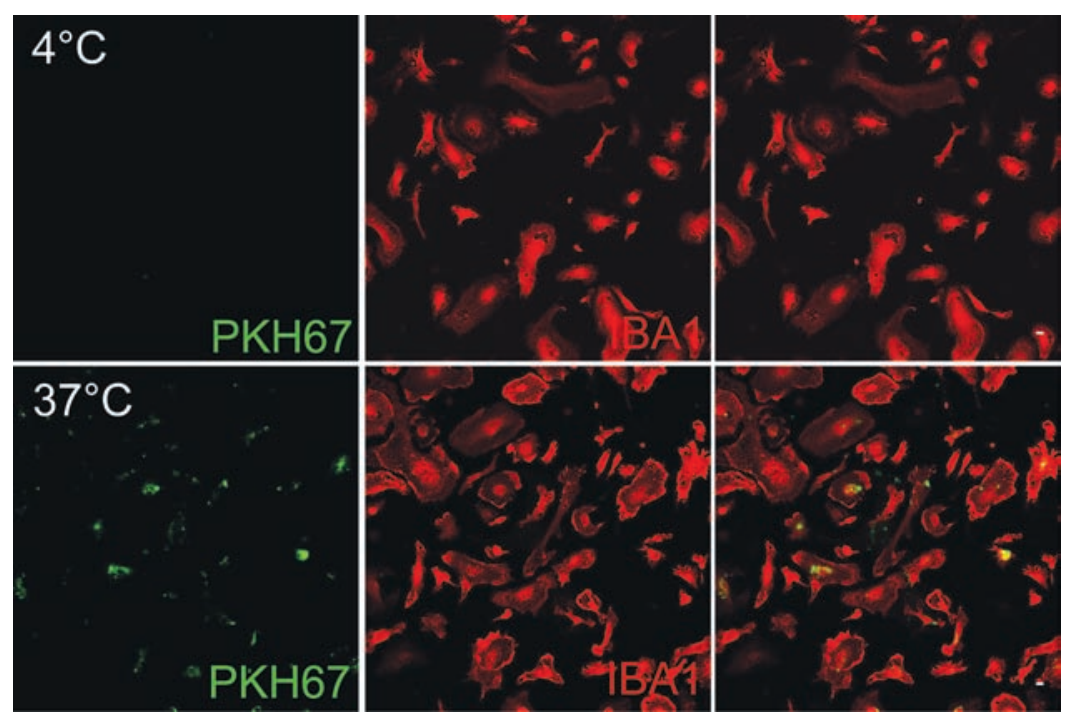

Figure 3.14: Exosomes do not show tethering on the plasma membrane of microglia at $4^{\circ}$ C. Exosomes were purified, labeled with $\mathrm{PKH} 67$ (green) and incubated with primary microglia either at $4^{\circ} \mathrm{C}$ or at $37^{\circ} \mathrm{C}$ for $1 \mathrm{~h}$. After washing three times with PBS, cells were fixed and stained for lba1 (red). Scale bar, $10 \mu \mathrm{m}$.

data supports our conclusion that exosome internalization is based on macropinocytosis, since it does not depend on a close interaction of cargo with the receptor.

\subsubsection{Uptake of exosomes is reduced in the presence of phos- phatidylserine containing liposomes}

The lipid phosphatidylserine, normally found in the inner leaflet of the plasma membrane is one of the key players during phagocytosis of apoptotic bodies [224]. In case of apoptotic cell death, this lipid gets actively exposed to the extracellular space and is recognized by phagocytes prior to phagocytotic clearance $[155,156]$.

Previous studies have shown an enrichment of phosphatidylserine in the exosomal membrane, which is facing the extracellular environment [201, 225, 226]. To verify these observations, we purified exosomes and confirmed the exposure of phosphatidylserine by annexin-V labeling using electron microscopy and flow cytometric approaches (see Figure 3.15 A, B). 
A

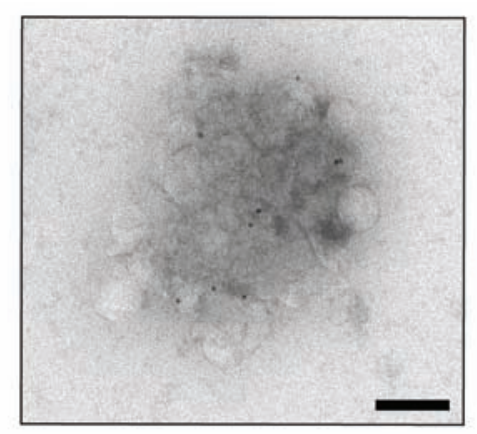

annexinV

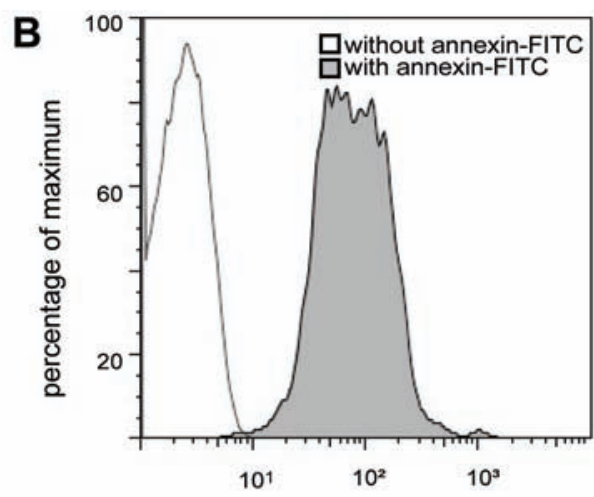

annexin-FITC fluorescence intensity

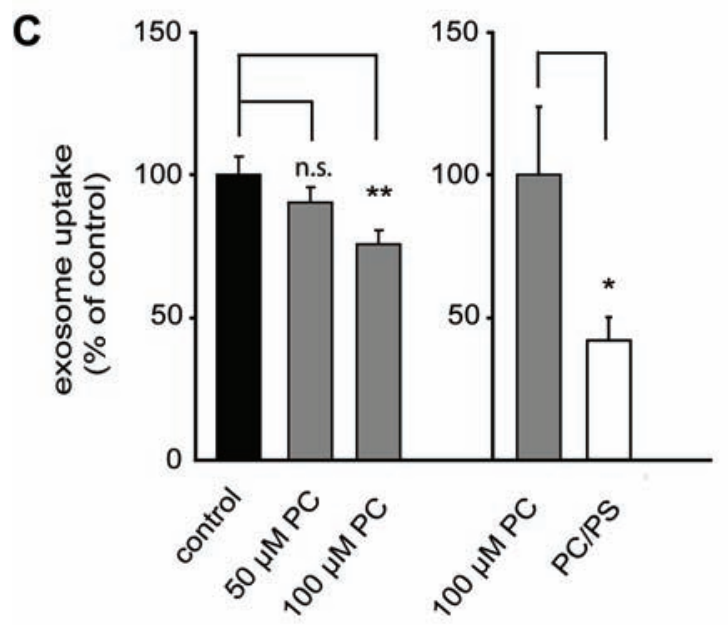

Figure 3.15: Uptake of exosomes is reduced in the presence of phosphatidylserinecontaining liposomes. (A) Surface-exposed phosphatidylserine (PS) was detected with annexinV-biotin and streptavidin-coupled gold particles on purified primary exosomes by electron microscopy. Scale bar, $200 \mathrm{~nm}$. (B) Exosomes were bound to the surface of latex beads, either stained or not with annexin- $V$ and analyzed by flow cytometry. (C) Liposomes containing phosphatidylcholine (PC) or a mixture of phosphatidylcholine and phosphatidylserine (PC/PS) were coincubated with exosomes and EOC-20 cells for 2 hours and uptake of exosomes was quantified. Values are given as the mean $+/$ - SEM $(n=3$; $\left.{ }^{*} p<0.05,{ }^{* *} p<0.01\right)$.

To test the implication of phosphatidylserine in the internalization event of exosome uptake, we prepared liposomes either containing a mixture of phophatidylcholine and phosphatidylserine or phosphatidylcholine alone. We found, that high 
concentrations of liposomes containing phosphatidylcholine significantly reduced exosome internalization. Liposomes comprised of a mixture of phosphatidylserine and phosphatidylserine, however, lead to a considerably higher decrease of exosome internalization (see Figure 3.15, C). These observations illustrate that exosomes compete with liposomes for their macropinocytic uptake, while this competition is more efficient if liposomes contain phophatidylserine.

\subsubsection{Exosome internalization is increased in presence of recom- binant milk-fat globulin E8}

Previous studies on exosomal composition showed that exosomes contain the protein MFGE8, a soluble molecule that binds to phosphatidylserine (PS) and acts as a bridging molecule linking PS to phagocytic receptors [33, 44, 227]. To analyze the expression of MFGE8 in the CNS we stained brain sections of adult NMRI mice with antibodies directed against glial marker proteins, as well as MFGE8. We found that MFGE8 signals partially colocalized with astrocytic marker protein GFAP (see Figure 3.16). 


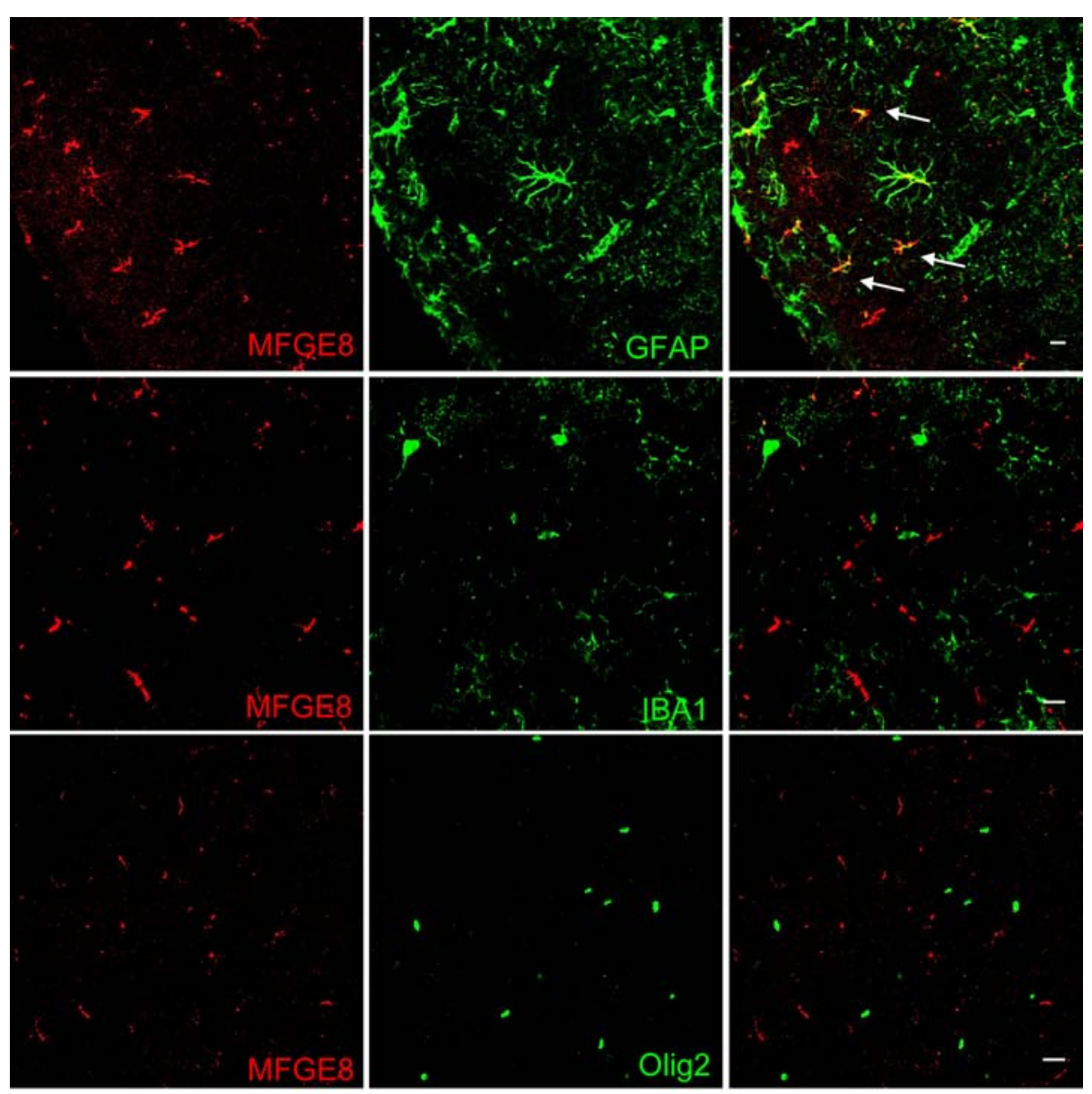

Figure 3.16: MFGE8 is expressed by astrocytes in the murine brain.

Sections of adult NMRI mice were immunolabeled for glial marker proteins Iba1, GFAP and Olig2 (green) as well as for MFGE8 (red). Images taken of the corpus callosum show MFGE8 signal partially colocalizing with the astrocytic marker GFAP, while no colocalization was observed with microglia marker Iba1 and oligodendroglial marker Olig2. Arrows indicate cells which colocalize with MFGE8 signal. Scale bar, $10 \mu \mathrm{m}$.

To study the influence of MFGE8 on exosome internalization, we labeled purified exosomes with PKH67 (green) and incubated them with recombinant mouse MFGE8 protein for 10 min before incubating them with EOC-20 cultures. Microscopic analysis revealed that the presence of recombinant MFGE8 significantly increased the amount of internalized exosomes in vitro (see Figure 3.17). 

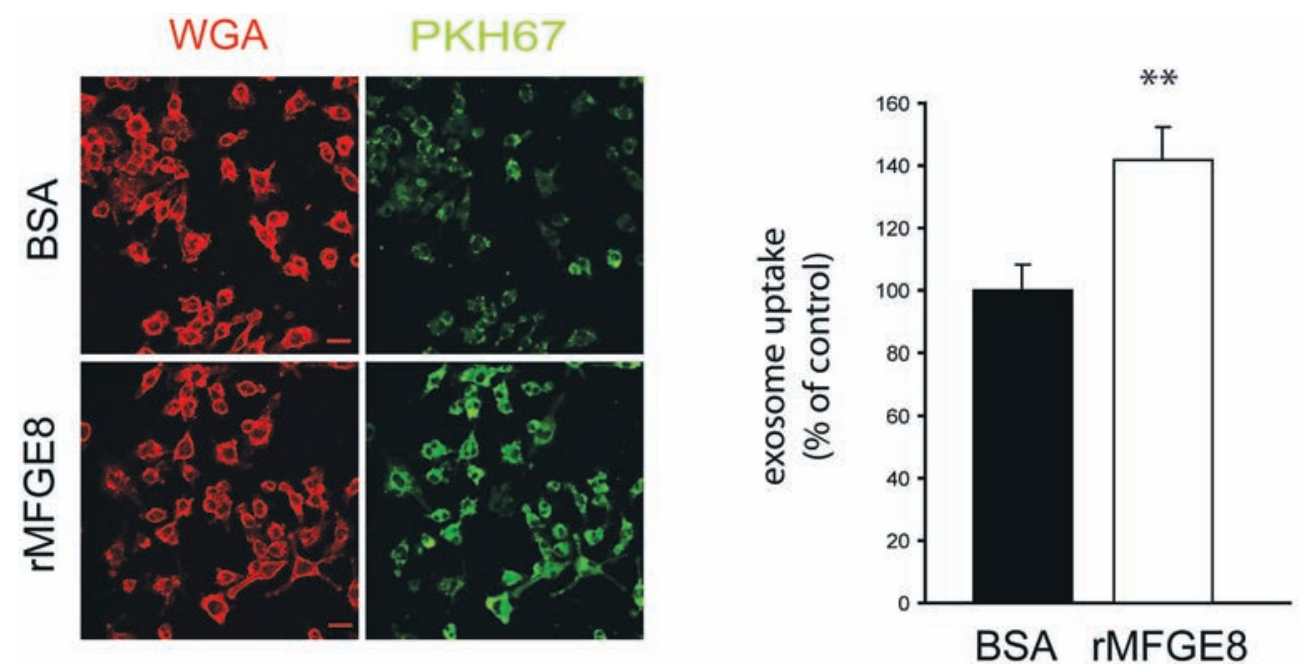

Figure 3.17: Recombinant MFGE8 facilitates exosome internalization into microglia. Exosomes were purified, labeled with PKH67 (green) and incubated with $100 \mathrm{ng} / \mathrm{mL}$ recombinant mouse MFGE8 protein for $10 \mathrm{~min}$ before addition to EOC-20 cultures on coverslips and further incubation in a humid chamber for $2 \mathrm{~h}$ at $37^{\circ} \mathrm{C}$. Cells were washed with PBS, fixed and stained with WGA (red). Exosome uptake was determined by microscopy and image analysis. Exosome internalization was increased in presence of recombinant MFGE8. Values represent means ${ }^{+} /$- SEM of three independent experiments.

\subsubsection{Fluid phase uptake versus phagocytosis - Internalization of dextran and myelin}

We continued to characterize the exosome internalization pathway by comparing it to the internalization of fluid phase marker dextran and purified myelin. While it is widely appreciated that dextran is internalized by macropinocytosis, myelin is described to be cleared by phagocytosis [207, 221, 228]. To characterize the distinct pathways, we purified and labeled exosomes and incubated them with FITC-dextran and primary microglia. After 10 min we observed partial co-localization of exosomes and FITC-dextran, while the majority of dextran and exosome signals showed segregated, vesicular distribution within the cell (see Figure 3.18). Quantification revealed that $9.5 \%$ of dextran-positive vesicles were also positive for exosomes, while 


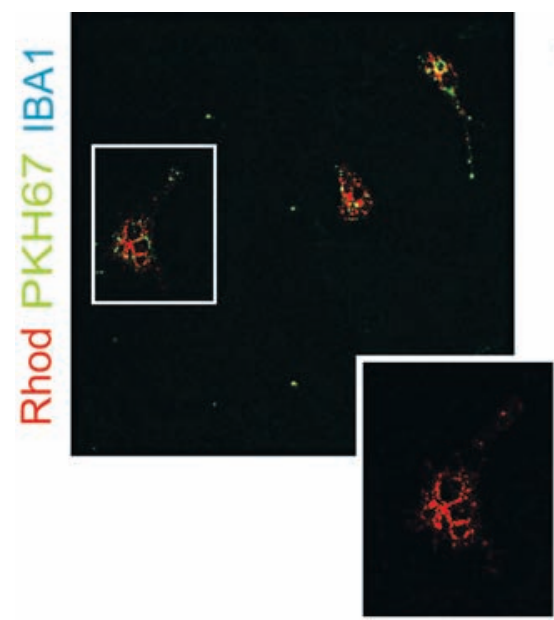

exosome-positive vesicles

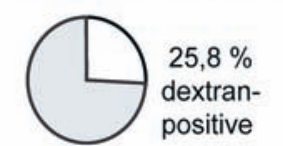

$74,2 \%$

dextran-negative

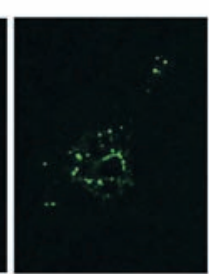

dextran-positive vesicles

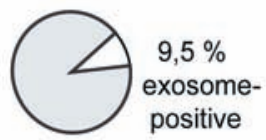

$90,5 \%$

exosome-negative

Figure 3.18: Exosomes and rhodamine-dextran are partially co-internalized by microglia. Exosomes were purified from the oligodendroglial cell line, Oli-neu, labeled with the dye PKH67 (green) and incubated together with rhodamine-dextran (red) for $10 \mathrm{~min}$ at $37^{\circ} \mathrm{C}$ on primary microglial cultures. For microscopic analysis, microglia were visualized using lba1 antibody (cyan). Exosomes partially co-localize with the fluid phase marker rhodamine-dextran. Internalization was quantified from 3 independent experiments. Values are given as means.

25.8\% of exosome-positive vesicles were additionally positive for FITC-dextran. Furthermore, we compared exosome internalization to myelin phagocytosis. We purified myelin from adult NMRI mice and confirmed its purity by Western blot analysis. These experiments revealed the abundance of mayor myelin protein PLP, while marker proteins of other glial cell types (Iba1 and GFAP) and cell organelles were absent. Only the presence of $\beta$ III-Tubulin revealed a slight contamination with neuronal components (see Figure 3.19). After crosslinking of FITC to myelin proteins, we performed internalization assays on microglial cells. As for oligodendroglial exosomes, we were able to detect internalization of FITC-myelin by microglia, verified by PLP co-stainings and transport of myelin to the late endosome/ lysosome (see Figure 3.19, B and Figure 3.20). Co-internalization studies with FITC-myelin and PKH28 labeled exosomes revealed a partial co-localization after internalization by microglia (see Figure 3.21). Whether the uptake occurs by different pathways that conveys into the same population of lysosomes remains to be elucidated. 
A

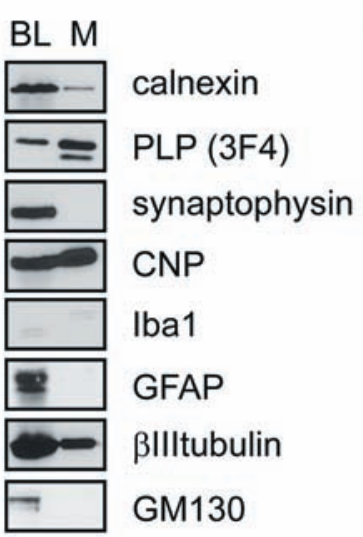

B

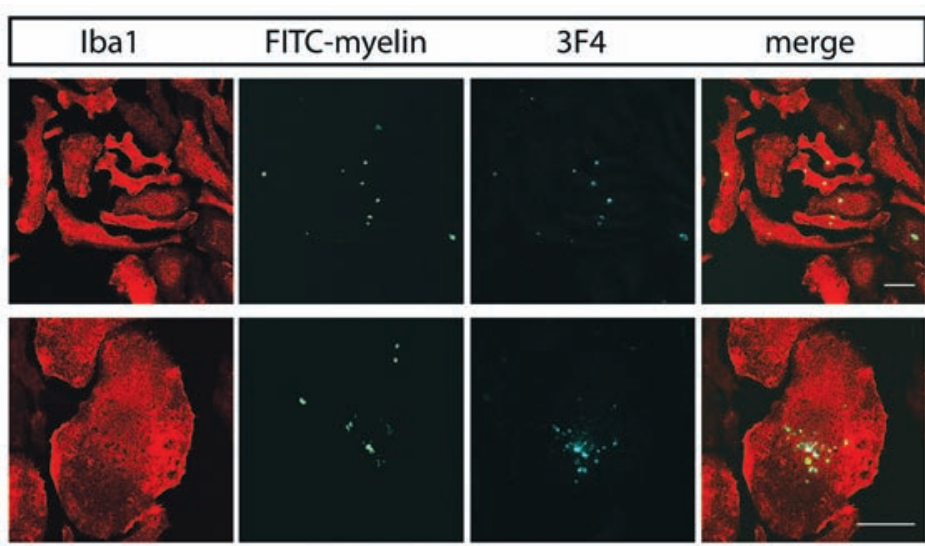

Figure 3.19: Microglia internalize FITC-labeled myelin. (A) Myelin was isolated from eight weeks old NMRI mice brains and $3 \mu$ g myelin (M) or $1 \mu \mathrm{L}$ brain lysate $(\mathrm{BL})$ were loaded onto the gel. Myelin protein composition was analyzed by Western blot with antibodies recognizing calnexin, PLP, synaptophysin, CNP, Iba1, GFAP, $\beta$ Illtubulin and GM130. (B) Myelin was isolated as shown in A and covalently labeled with fluoresceinisothiocyanate (FITC). 1 to $2 \mu \mathrm{g}$ of FITC-myelin (green) were incubated with primary microglial cultures for $2 \mathrm{~h}$ at $37^{\circ} \mathrm{C}$, cells were fixed, permeabelized and stained with microglial marker lba1 (red) and antibodies against major myelin protein PLP (3F4,cyan). FITC-myelin was internalized by microglia and co-localized with myelin protein PLP. Scale bars, $20 \mu \mathrm{m}$. 


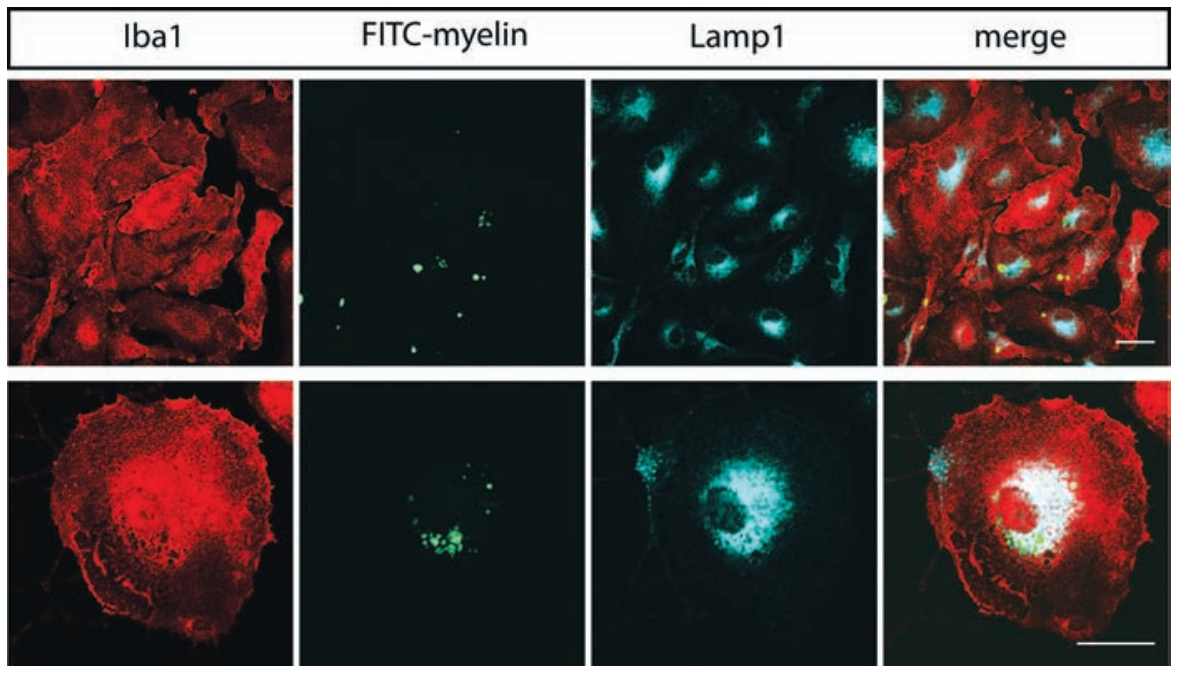

Figure 3.20: Internalized FITC-myelin is found in the late/endosomal compartment of microglia. 1 to $2 \mu \mathrm{g}$ of FITC-myelin (green) were incubated with primary microglial cultures for $2 \mathrm{~h}$ at $37^{\circ} \mathrm{C}$. Cells were fixed and permeabelized. Microglia were recognized with lba1 (red) and the late endosome/ lysosome with antibodies against Lamp-1. FITCmyelin was internalized by microglia and transported to the late endosome. Scale bars, $20 \mu \mathrm{m}$. 


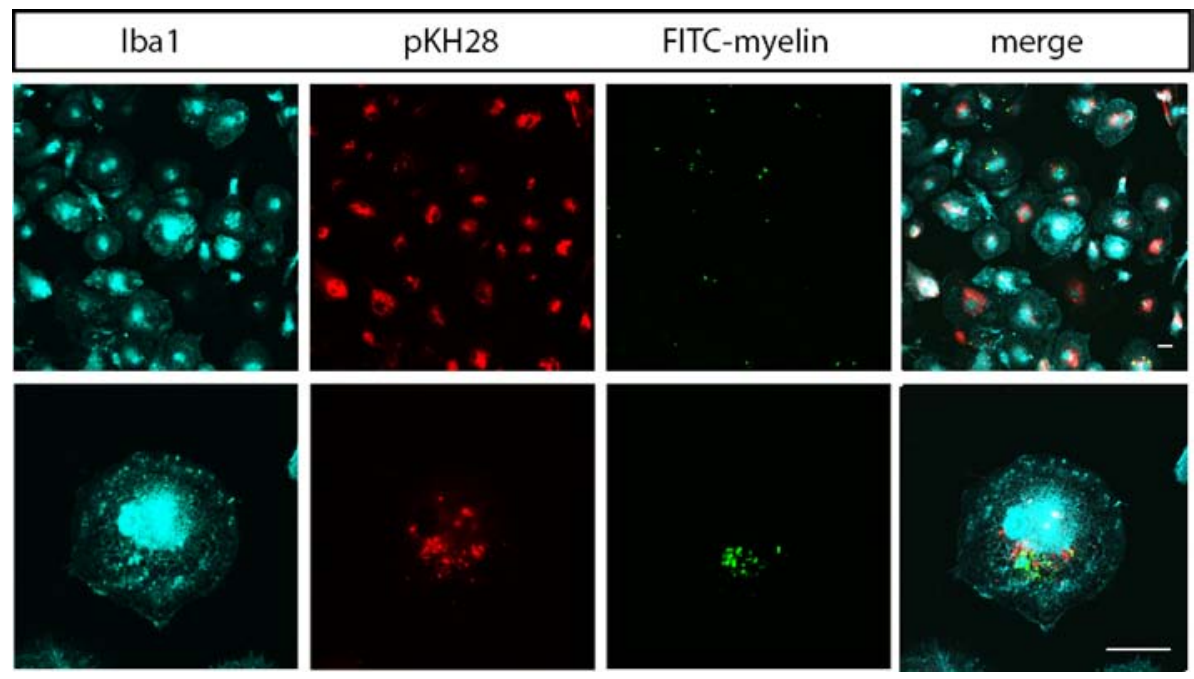

Figure 3.21: Exosomes and FITC-myelin partially co-localize after internalization by microglia. Exosomes of $1 \times 10^{6}$ Oli-neu cells were purified, labeled with $\mathrm{PKH} 28$ (red) and incubated together with $1 \mu \mathrm{g}$ of FITC-myelin (green) on primary microglial cultures for $2 \mathrm{~h}$ at $37^{\circ} \mathrm{C}$. After fixation, cells were stained with microglial marker Iba1 (cyan). Exosomes and FITC-myelin partially co-localize after internalization by microglia. Scale bars, $20 \mu \mathrm{m}$. 


\subsection{Exosome uptake occurs in an immunologically silent manner.}

Recent studies by Nimmerjahn and colleagues have clearly demonstrated that the previously used term "resting" microglia in an uninjured brain does not reflect anymore the active role of microglia in the surveillance of the central nervous system (CNS) [111]. While the cell body may remain fixed in location, microglial processes were shown to continuously sample the brain parenchyma [111]. However, in response to injury or pathogens, microglia respond with integrative signaling pathways associated with the production and release of pro-inflammatory and antiinflammatory cytokines and chemokines [117, 229, 230].

To analyze whether the contact or internalization of exosomes leads to a change of the "surveying status" of microglia, we tested the release of immunoregulatory mediators by microglia. In this experiment, we incubated primary microglia with different concentrations of exosomes and accessed the release of several prominent cytokines and chemokines via ELISA (see Figure 3.22). The results showed that important pro-inflammatory cytokines (TNF $\alpha$, IL-6 and IL-12) were not induced after internalization of exosomes, while incubation with lipopolysaccharide lead to a robust induction (see Figure 3.22, A). Similar results were obtained for the pro-inflammatory chemokine $\mathrm{KC}$, while the anti-inflammatory cytokine IL-10 release remained also unaffected (Figure 3.22, B). Interestingly, the release of both chemokines MIP1 $\alpha$ and RANTES was slightly induced, but only in response to high exosome concentrations and compared to LPS to a much lower degree (Figure 3.22, C). Remarkably, also the LPS induced release of pro-inflammatory cytokines and chemokines was not influenced by the addition of exosomes. 
A
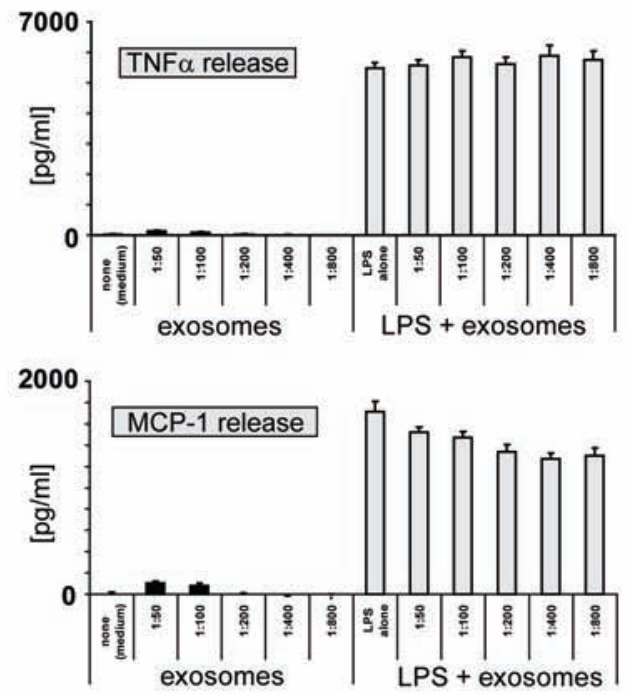

B

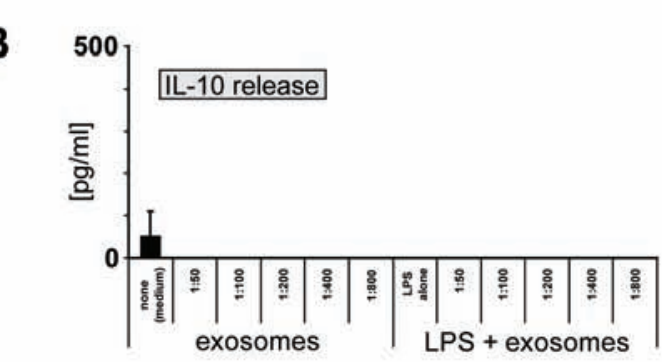

C

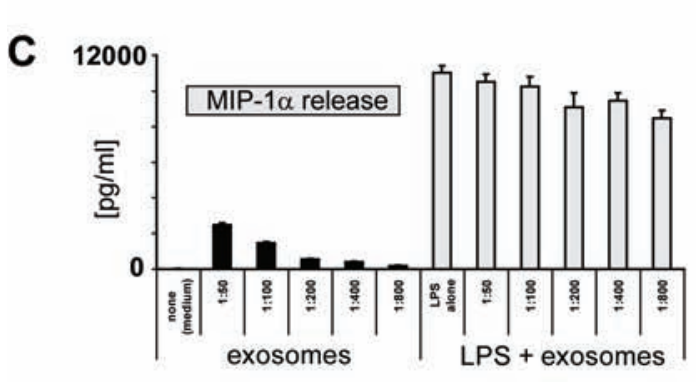

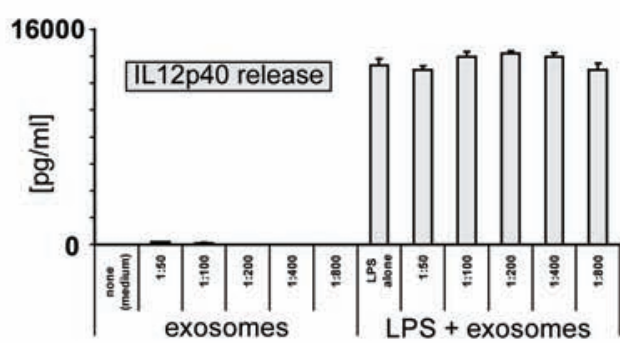

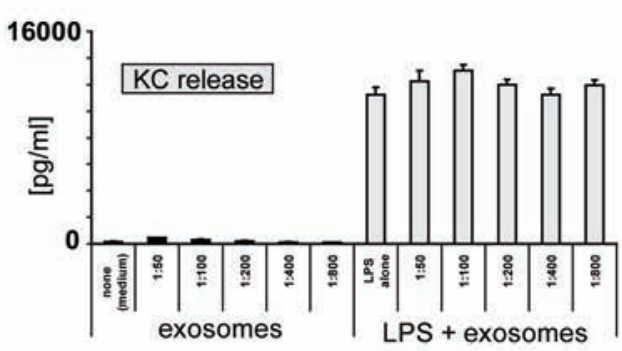

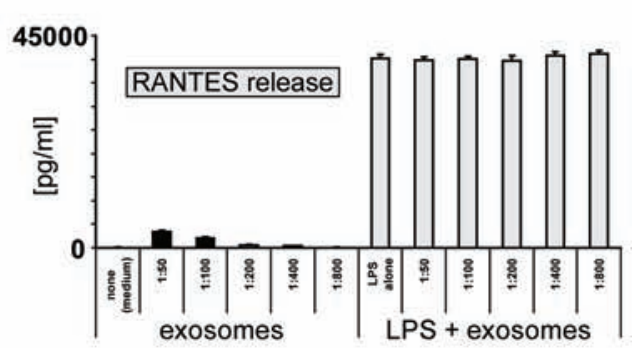

Figure 3.22: Exosomes do not lead to pro-inflammatory nor anti-inflammatory cytokine release of microglia. Purified exosomes (from $2 \times 10^{7}$ cells, resuspended in $100 \mu \mathrm{L}$ PBS; diluted as indicated) were incubated with primary cultures of microglia $(15,000$ cells/well) in the presence or absence of LPS $(100 \mathrm{ng} / \mathrm{mL})$ for $16 \mathrm{~h}$ and the cytoand chemokine release profile was determined by ELISA. Exosomes induce a slight release of MIP $1 \alpha$ and RANTES, but do not change the cytokine profile of microglia. Data are means ${ }^{+} /$- SEM of triplicates of two independent experiments. 


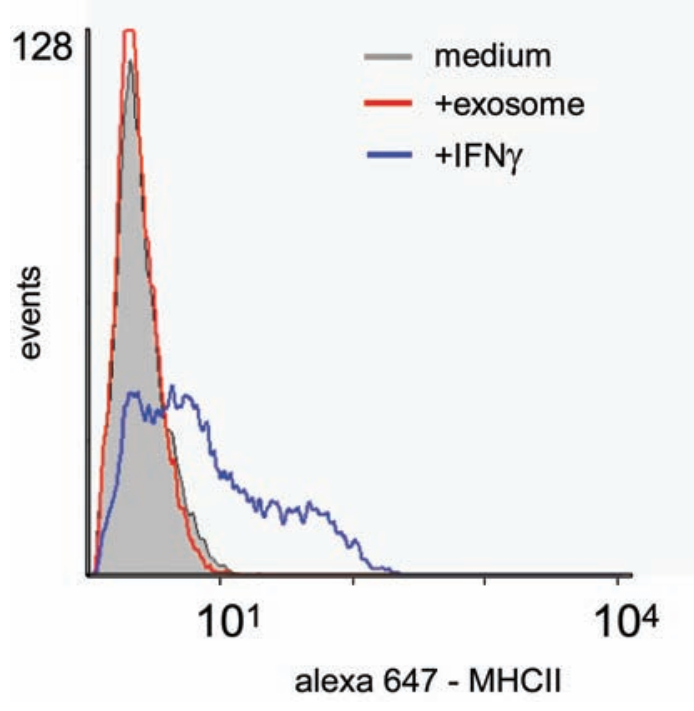

Figure 3.23: Exosomes do not alter IFN $\gamma$ induced MHCIl expression of microglia. Cell culture medium alone or containing either purified exosomes or IFN $\gamma$ was added to microglial cultures. Mayor histocompatibility complex II (MHCII) expression was determined on the surface of microglia by flow cytometric analysis analysis.

In addition to these findings, we found that injection of labeled exosomes into the central nervous system of Cx3cr1/eGFP mice did not induce morphological changes of microglial cells, as observed with two-photon microscopy (data not shown). Moreover, cells did not enforce their motility towards the injection site, as described for a laser lesion set in the spinal cord [111]. Beside the influence of exosomes on the release of immunoregulatory molecules, we tested whether exosomes change the expression status of major histocompatibility complexes (MHC) class II. After stimulation with exosomes for $24 \mathrm{~h}$, we did not see an induction of MHCII, while stimulation with IFN $\gamma$ resulted in an upregulation of MHCII expression in microglia as shown by flow cytometric measurements (see Figure 3.23). Overall we concluded, that exosome treatment and internalization by microglia did not change their activation status.

Since oligodendrocyte derived exosomes contain myelin proteins like PLP and MOG, they might carry potential candidate antigens implicated in the autoimmune response of multiple sclerosis $[231,232]$. To test whether exosomes can transport 
antigens to microglia for presentation to $\mathrm{CD} 4^{+} \mathrm{T}$ cells, we used MOG-specific TCRtransgenic mice [208]. These mice show expression of a $\mathrm{T}$ cell receptor recognizing the MOG aa35-55 peptide. For the experiment, primary microglia were stimulated with IFN $\gamma$ and treated with MOG, MOG aa35-55 peptide or with MOG-positive exosomes. After addition of $\mathrm{CD}^{+}{ }^{+} \mathrm{T}$ cells, cells were incubated for five days before analysis. The activation of MOG-specific CD4 ${ }^{+} \mathrm{T}$ cells was measured by the release of IL-2 into the culture medium (see Figure 3.24).

We found that unstimulated microglia treated with high doses of MOG aa35-55 peptide were not capable of $\mathrm{CD}^{+} \mathrm{T}$ cell activation (see Figure 3.24). However, treatment of unstimulated antigen presenting cells, in this case splenocytes, resulted in a strong IL-2 release (see Figure 3.24, B). Strikingly, after stimulation of microglia with IFN $\gamma$ before adding MOG aa35-55 peptide, a significant release of IL-2 was detected, which was comparable to the $\mathrm{T}$ cell response to splenocytes. A similar release was found when recombinant full length MOG was added to IFN $\gamma$ stimulated microglia (see Figure 3.24, A). 

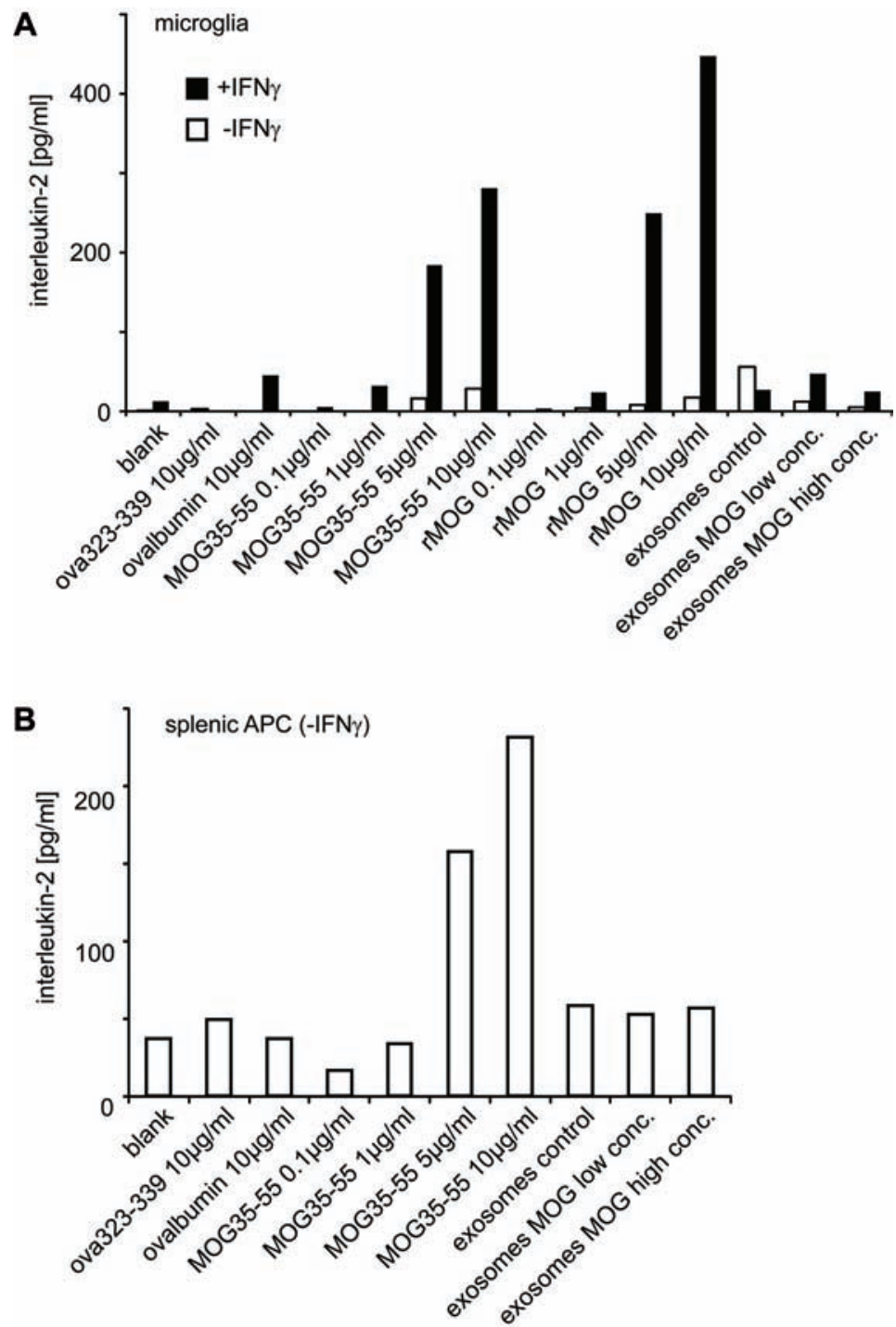

Figure 3.24: Exosomal proteins are not presented via MHCll. Recombinant extracellular myelin oligodendrocyte glycoprotein (MOG aa1-125), MOG peptide aa35-55, ovalbumin, ovalbumin peptide aa323-339 and purified exosomes in low (from $1.5 \times 10^{6}$ cells) or high (from $3 \times 10^{6}$ cells) concentration were added to primary microglia prepared from newborn mice $\left(5 \times 10^{4}\right.$ cells/well) or splenocytes $\left(2 \times 10^{5}\right.$ cells/well) together with purified MOG-specific 2D2 CD4 ${ }^{+}$T cells $\left(1 \times 10^{5}\right.$ cells/ well $)$ and incubated for $72 \mathrm{~h}$. Microglia were either prestimulated with IFN $\gamma$ overnight or not. A MOG-specific T cell response was determined by IL-2 secretion in the culture supernatants using enzyme-linked immunosorbent assay system. Data are displayed as the mean of duplicates showing one representative out of three independent experiments. 
To analyze the antigen transfer ability of exosomes, we purified exosomes from Oli-neu cells overexpressing full length MOG, and added them to IFN $\gamma$ stimulated microglia. We did not detect IL-2 release, therefore we concluded that MOG-positive exosome were not capable to induce a MOG-specific $\mathrm{T}$ cell activation (see Figure $3.24, \mathrm{~A})$. These findings could be just due to low antigen levels transfered by exosomes. However, one alternative explanation for the lack of $\mathrm{T}$ cell activation was found in continuative experiments examining the exosome internalization of IFN $\gamma$ -stimulated microglia (see below).

\subsection{Inflammatory stimuli downregulate macropinocyto- sis of exosomes in microglia}

Since microglia use macropinocytosis for the internalization of exosomes, we tested whether IFN $\gamma$ stimulation prior to exosome internalization experiments alters the macropinocytic activity of the cell. In fact, flow cytometric analysis revealed a decrease in exosome uptake, which could also be shown for the endocytosis of fluid phase marker, dextran, after IFN $\gamma$ treatment (see Figure 3.25, A). Similar results were found after stimulation with LPS (see Figure 3.25, B). We concluded, that the macropinocytotic activity of microglia is down-regulated by inflammatory signals such as IFN $\gamma$ and LPS.

In the preceding experiments (see Figure 3.23), we observed that only a sub-population of microglia shows an increase of MHCII surface expression, while another fraction remains MHCII negative. Likewise, cultures stimulated with IFN $\gamma$ show differential expression of costimulatory molecules B7-1 and B7-2 (see Figure 3.27). Finally, internalization experiments with IFN $\gamma$ stimulated microglia revealed that exosomes are preferentially internalized by microglia with low levels of MHC II expression (see Figure 3.26).

To summarize, our results demonstrate that oligodendroglial exosomes are mainly internalized by microglia with high macropinocytoptic capacities. This internalization process can be altered by inflammatory signals, shifting exosome clearance 
A

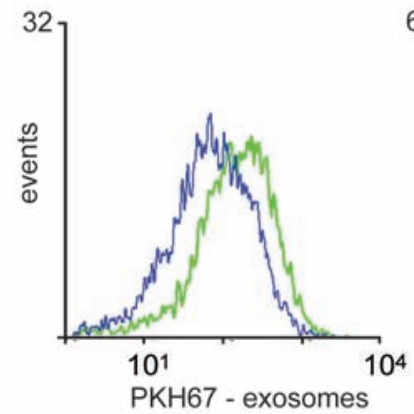

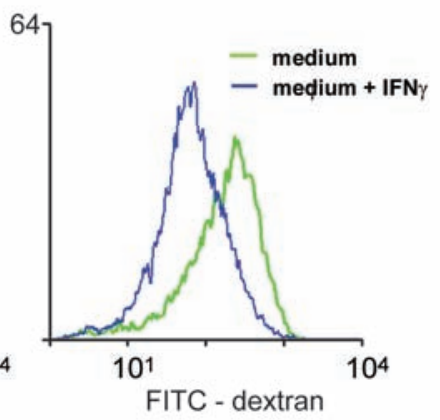

B

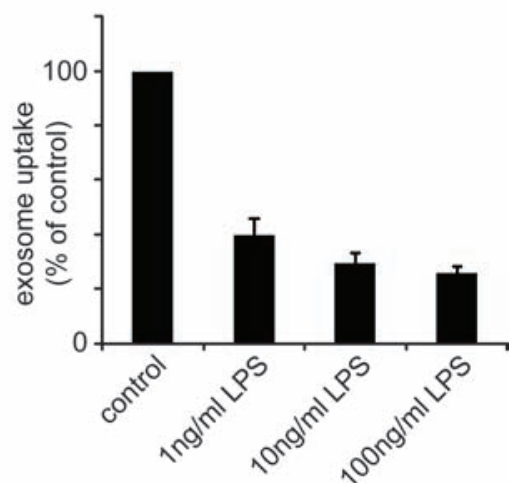

Figure 3.25: Inflammatory stimuli downregulate macropinocytosis of exosomes in microglia. (A) Primary microglia were treated for $24 \mathrm{~h}$ with IFN $\gamma$ or left untreated. Purified exosomes labeled with the dye PKH67 (green) or 40 kD FITC-conjugated Dextran were incubated for $2 \mathrm{~h}$ with primary microglia (exosomes from $1 \times 10^{7}$ Oli-neu cells/ well) and internalization was determined by flow cytometry. (B) Primary microglia were treated for $24 \mathrm{~h}$ with different concentration of LPS and uptake of purified exosomes was determined by microscopy and image analysis. Values represent means ${ }^{+} /$- SEM of three independent experiments.

activity to a subpopulation of microglia and raising the possibility of identifying heterogeneity in the microglia population of the CNS. 

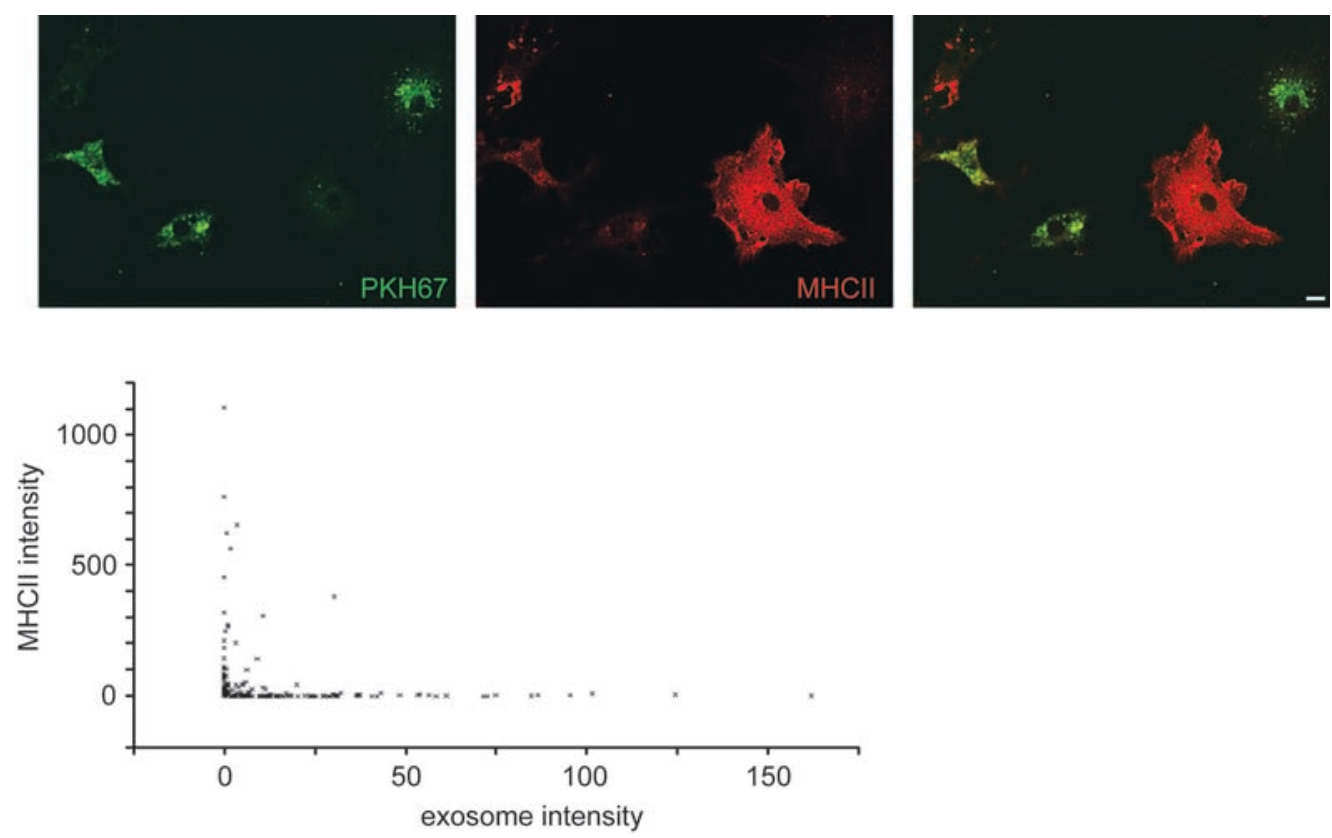

Figure 3.26: Microglia expressing MHCII show decreased exosome internalization. Microglia were treated for 8 to $12 \mathrm{~h}$ with IFN $\gamma$ before adding purified labeled exosomes to the cells for $2 \mathrm{~h}$. Microglia were fixed, permeabilized and stained for MHCll. Average fluorescence intensities were determined for exosomes (green) and $\mathrm{MHCll}$ (red) by microscopy and images were analyzed from two independent experiments, as depicted in the graph. 

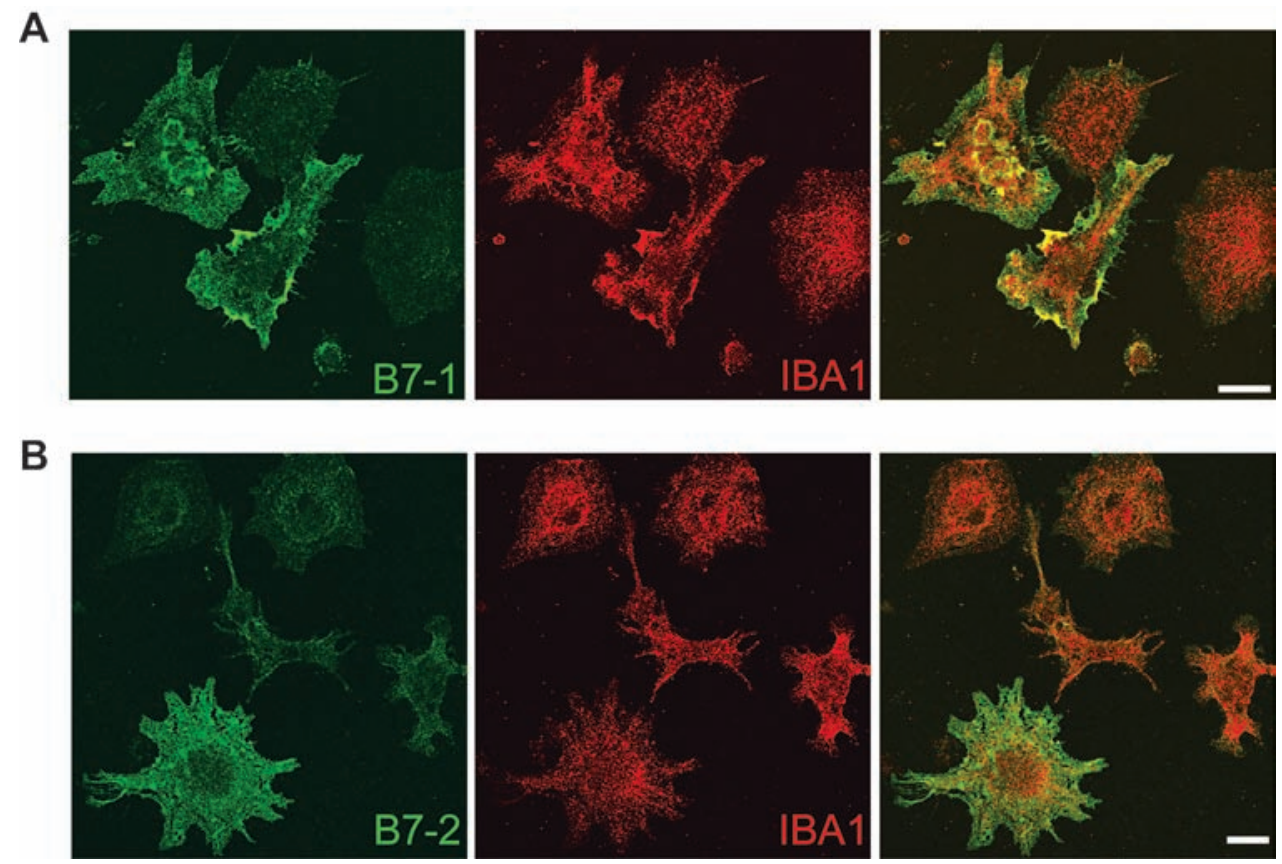

Figure 3.27: IFN $\gamma$ stimulated microglia show differential expression of B7-1 and B7-2. Primary cultures of microglia were stimulated with $100 \mathrm{ng} / \mathrm{mL} \mathrm{IFN} \gamma$ for $24 \mathrm{~h}$ and stained for (A) CD80 (B7-1) and (B) CD86 (B7-2) and lba-1. An upregulation of B7-1 and B7-2 was observed in a subpopulation of microglia. Scale bar, $20 \mu \mathrm{m}$. 


\subsection{Functional heterogeneity in microglial population of the CNS?}

Lawson and colleagues observed already in 1990 that the regional density and shape of microglia varies depending on the brain region [100]. However, recent findings demonstrated that differences in apparent microglial phenotype extend beyond simple locational divisions [101, 233-235]. In our study, putative subpopulations of microglia are defined by their capacity to clear exosomes while lacking MHCII expression and vice versa.

To study microglial heterogeneity, we started to quantify the abundance of both microglial subtypes present after IFN $\gamma$ stimulation. For this, primary microglial cultures were stimulated with IFN $\gamma$ for $48 \mathrm{~h}$ and processed for flow cytometric analysis. Quantification of microglial subtypes revealed that 30 to $45 \%$ of microglia become MHC II positive upon IFN $\gamma$ stimulation, while the remaining cells stay MHCII negative (see Figure 3.28). The MHC II expression reached a peak after $48 \mathrm{~h}$ of stimulation (data not shown). Besides these in vitro findings, we wanted to confirm that microglia can express MHCII in vivo. After injection of IFN $\gamma$ into the hippocampus of anathesized adult mice, we were indeed able to detect MHCII expression in microglial cells (see Figure 3.29). 

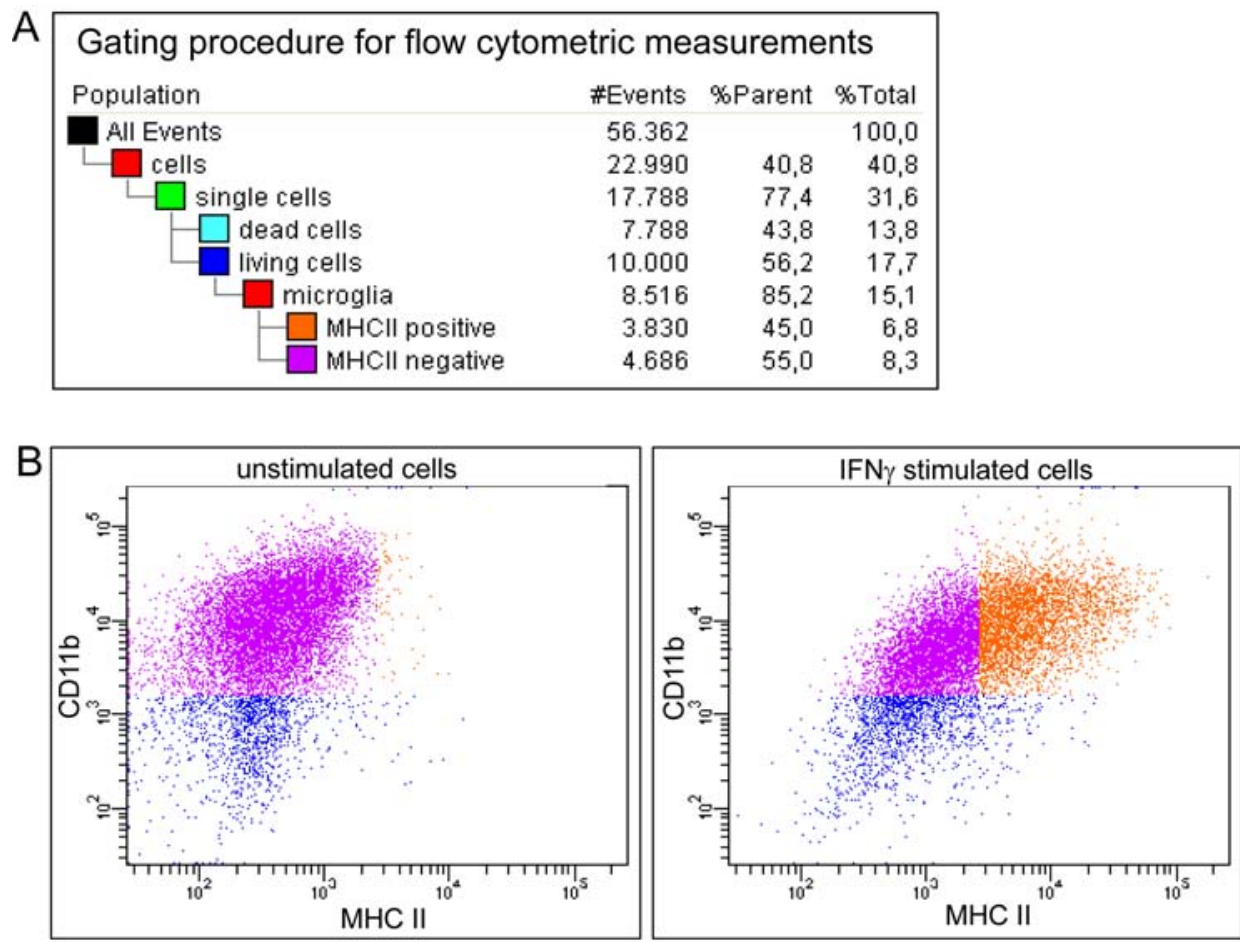

Figure 3.28: Quantification of IFN $\gamma$ inducible MHCIl expression of microglia in vitro. Primary glial cultures were prepared of PO mice and incubated for two weeks, before they were shaken from the astrocytic cell layer and further kept for two days prior to stimulation with $100 \mathrm{ng} / \mathrm{mL} \mathrm{IFN} \gamma$. After $48 \mathrm{~h}$, cells were prepared for flow cytometric analysis, using antibodies directed against microglial marker CD11b and MHCII. (A) Gating procedure for the flow cytometric analysis of microglia cells. After exclusion of cell debris and apoptotic cells, CD11b positive microglial cells (red) were analyzed for expression of MHCII (orange). Quantitative data is shown on the right. (B) Scatter plots of flow cytometric data showing cell populations stimulated with IFN $\gamma$ or not. Cells positive for CD11b are shown in pink, while MHCII positive microglial cells are shown in orange. Data are displayed as one representative out of 15 independent experiments. 


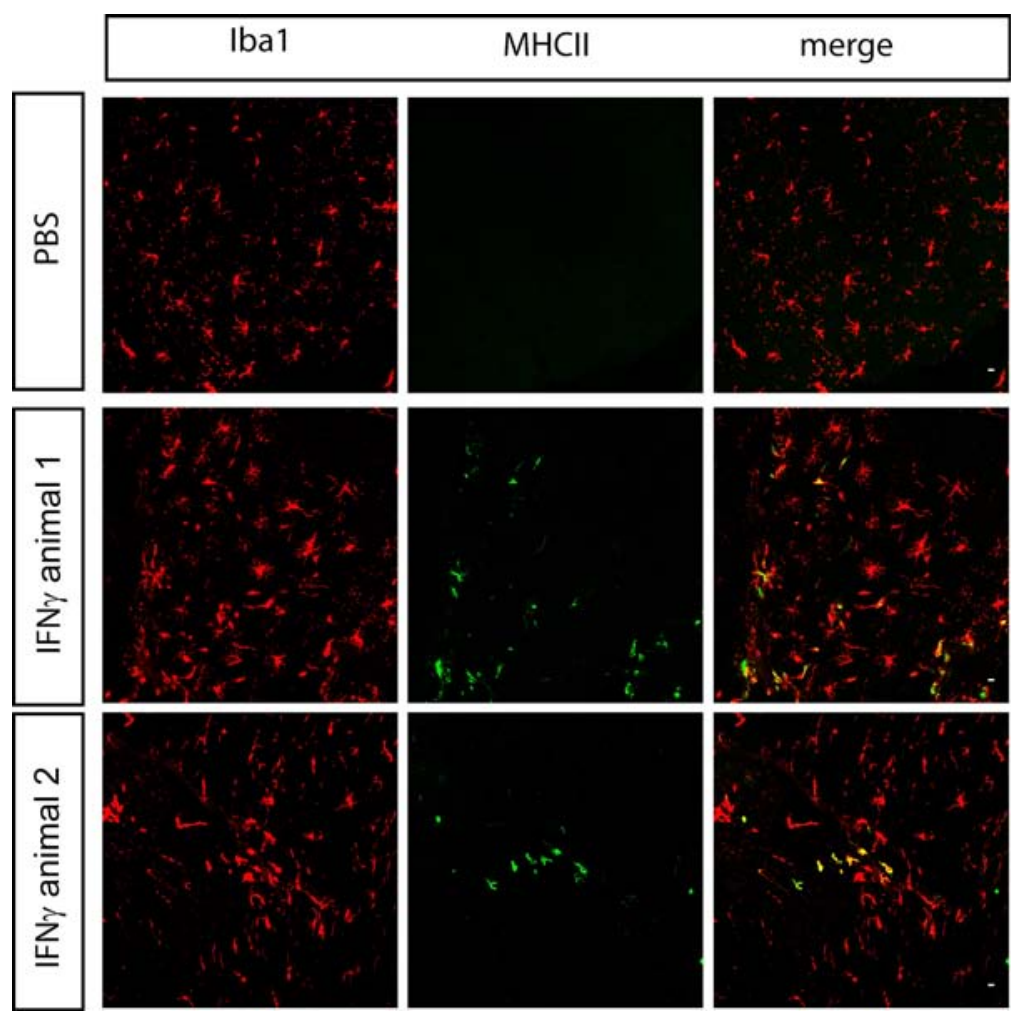

Figure 3.29: Stimulation of MHCII expression in vivo by injection of IFN $\gamma$. After injection of IFN $\gamma$ into the hippocampus, adult NMRI mice were kept for $24 \mathrm{~h}$ to allow diffusion of IFN $\gamma$. Mice were sacrificed and brain sections were stained for microglial marker Iba1 and MHC II, respectively. Microscopic analysis of the hippocampal region showed partial upregulation of MHCII expression in microglia. However, also other cell types showed weak expression of MHCII. Scale bar, $10 \mu \mathrm{m}$. 


\section{Chapter 4}

\section{Discussion}

The aim of this work was to follow the fate of exosomes secreted by oligodendrocytes. We found that microglia selectively and efficiently internalize exosomes by a macropinocytotic mechanism. Uptake of exosomes occurs without inducing a concomitant pro-inflammatory response and without microglia supporting a MOGspecific $\mathrm{CD}^{+} \mathrm{T}$ cell response. Stimulation of microglia by $\mathrm{IFN} \gamma$ leads to an upregulation of MHCII in a subpopulation of microglia. However, internalization of exosomes occurs predominantly in a subpopulation of MHCII-negative cells. Removal of exosomes from the extracellular space may occur in microglia that are mainly engaged in the clearance of material. Moreover, the activation of microglia by IFN $\gamma$ or LPS results in a decrease of the macropinocytotic activity.

\subsection{Exosomes are selectively internalized by microglia - in vitro and in vivo}

Our studies showed that exosomes are preferentially internalized by microglial cells in vitro. Astrocytes, oligodendrocytes and neurons did not show exosome binding or internalization of oligodendroglial exosomes. After internalization exosomes are found in the late endosomal compartment of the microglial cell and exosomal proteins are degraded over time. Similarly, DC-derived exosomes are found after $2 \mathrm{~h}$ in the late endosomal compartment of DCs [201]. When we injected oligodendrogliaderived exosomes into the spinal cord of adult mice, exosomes were internalized by 
microglia in vivo. Furthermore, we detected vesicular transport of exosomes towards the microglial cell body. Colocalization studies revealed that exosomes localize to the microglial late endosomal/ lysosomal compartment, as shown in vitro. Interestingly, Morelli and colleagues showed that intravenous injected allogenic exosomes could later on be located in macrophages and DCs of the splenic marginal zone [201]. As efficient phagocytes microglia offer various potential endocytic pathways which could account for the internalization of exosomes. This includes phagocytosis, a key pathway to remove pathogens as well as apoptotic cells. Since exosomes contain endogenous proteins their removal is likely to be mediated by receptors recognizing apoptotic bodies without engaging members of the Toll-like receptor family [167]. Numerous cell surface receptors, such as the scavenger receptors LRP1/CD91 and CD36, lectins, integrins, CD14 and C1q, have been shown to engage the apoptotic cell and promote phagocytosis [236]. Another putative pathway for exosome internalization is macropinocytosis, which differs from phagocytosis and other endocytic pathways through its unique susceptibility to changes of the lysosomal $\mathrm{pH}$ [218]. Furthermore, macropinocytosis can also be induced by growth factors, eminent in non-phagocytosing cells. In immature DCs, the constitutive macropinocytosis of immature dendritic cells has emerged as one prominent uptake pathway for antigen capture [237].

\subsubsection{Which key players of endocytic mechanisms are involved in exosome internalization?}

Exosomal internalization studies in presence of various pharmacological inhibitors revealed the role of multiple cellular proteins in the endocytic pathways of microglia. Our data showed that microglial exosome uptake was dependent on the activity of Rac1-GTPase. Rho-family GTPase Rac1 is one of the major regulators of the actin cytoskeleton, which undergoes dramatic rearrangements during endocytic events. In fibroblasts and dendritic cells, Rac1 activation is necessary to induce membrane ruffling during pinocytosis [238] or to maintain constitutive macropinocytosis, respectively [239]. Likewise, the compound cytochalasinD, which prevents actin polymerization by stabilizing its monomeric form [217], lead to a strong decrease in 
exosome internalization by microglia (see Figure 3.12).

Membrane ruffling in response to actin polymerization near the plasma membrane is not only due to activation of Rac1, but also to activation of other RhoGTPases Rho and Cdc42. Downstream mediators of these small family Rho GTPases are the EWASp/Scar proteins and the Arp2/3 complex. As the newly formed actin branch grows, the plasma membrane is forced out and extends into a membrane ruffle [240]. Our live-cell-imaging data revealed that similar membrane protrusions were observed during exosome internalization. Ultimately, exosomes were internalized accompanied by fusion of these membrane protrusions with the plasma membrane. Apart from large membrane protrusions, macropinocytic mechanisms based on membrane blebbing were described previously [221]. However, no blebbing of the plasma membrane was observed during exosome internalization by microglia (see Figure 3.12), which was consistent with the unaffected exosome internalization by myosinII-inhibitor blebbistatin (see Figure 3.12) [241].

Additionally, our data showed that the GTPase dynamin had a strong influence on exosome internalization. Dynamin is shown to form rings and decorates endocytic vesicles [242]. It is proposed that dynamin is directly responsible for pinching of vesicles of the membrane [215]. Dynamin could be involved in the scission of exosome-containing vesicles/macropinosomes from the plasma membrane. This hypothesis is strengthened by recent findings, showing dynamin2 activity during internalization of tumor-derived exosomes into macrophages, which was additionally dependent on actin [243].

Further experiments with a different set of inhibitors revealed, that drugs alkalizing the lysosomal compartment decreased the uptake of exosomes, which strongly supports a macropinocytotic mechanism of exosomal uptake (see Figure 3.12). One example is bafilomycin, a potent inhibitor of vacuolar-type, proton-translocating ATPases (v-ATPases) [219]. The v-ATPases pump protons into the lumen of endosomal membranes, resulting in the acidification of endosomes and lysosomes [219]. One possible explanation for the decrease of exosome internalization is a feedback 
mechansims preventing macropinocytosis, while lysosomal acidification and degradation is impaired. Moreover, recent studies revealed that ATPases are also necessary to sustain a certain submembranous $\mathrm{H}^{+}$concentration during actin polymerization. Thus, the presence of alkalizing drugs prevent the actin remodeling necessary to promote macropinocytosis [218] and ultimately the internalization of exosomes. Also, amiloride and chloroquine are shown to increase intralysosomal pH [220] and had a strong negative effect on exosome internalization by microglia.

\subsubsection{Is oligodendroglial exosome internalization a receptor medi- ated process?}

Since exosomes are specifically internalized by microglia, the mechanism of exosome uptake is likely to be mediated by specific receptors. These receptors and the respective ligands must be present both on exosomal and on microglial side. Like on all membrane vesicles, exosomes possess cellular adhesion molecules on the surface, which could facilitate their capture by recipient cells. For DCs, it was shown that incubation of exosomes with blocking antibodies specific for various integrins, adhesion molecules and tetraspanins, reduced exosome uptake by 5 to $30 \%$ [201].

The most prominent group of microglial receptors are those involved in phagocytosis. Our studies with antagonists of phagocytosis receptors revealed that neither scavenger receptors A nor B are involved in exosome internalization. In addition, the aminosugars glucosamine and mannoseamine were tested, which exert an inhibitory effect on phagocytosis via blocking lectin mediated phagocytosis [223]. However, both molecules did not influence the microglial clearance of exosomes. Similarily, antagonization of the low-density lipoprotein receptor had no effect on exosome internalization.

In contrast to our findings, studies on exosome capture of immature dendritic cell derived exosomes revealed a specific ligand-receptor interaction. The exosomal ICAM1 binds to the lymphocyte function associated antigen 1 (LFA1) on the surface of CD ${ }^{+}$dendritic cells and activated T cells [244, 245]. Additionally, integrin $\alpha 1$ and $\beta 2$ on exosomes are captured by extracellular matrix protein fibronectin or ICAM1 expressing cells [246, 247]. 


\section{Phosphatidylserine - a link to specific microglial receptors for exosome internalization?}

Beside protein receptors, exosomal membranous lipids could also serve as ligands for cellular receptors. As described by other groups earlier [248], our results confirmed that exosomes contain phophatidylserine in their outer membrane, which could be a putative ligand for the microglial receptors. In live cells, almost all the phosphatidylserine (PS) is confined to the inner leaflet of the plasma membrane, while apoptotic cells expose PS at the outer leaflet [249]. Blocking surface PS from recognition by its receptors results in a tremendous decrease in apoptotic cell engulfment by macrophages in vitro [172]. Interestingly, also viruses with the size of exosomes display phosphatidylserine on their viral membrane. In a process called apoptotic mimicry, vaccinia virus employs a PS-dependent macropinocytic mechanism to enter its target cell [221]. In our studies the presence of liposomes containing phosphatidylserine and phosphatidylcholine significantly decreased the internalization of exosomes in comparison to liposomes containing only phophatidylcholine. Similarly, Witting and colleagues showed that lipid vesicles enriched in phophatidylserine and O-phospho-L-serine interfered with the uptake of apoptotic neurons by microglia [250]. These findings indicate that PS is likely to be one of the key exosomal ligands involved in exosome clearance.

Phosphatidylserine can be bound by soluble bridging molecules, which in turn are recognized by phagocytic receptors. One of the bridging molecules described in PS related engulfment is milk fat globule epidermal growth factor 8 (MFGE8). MFGE8 binds to phosphatidylserine through its carboxy-terminal factor V/VIII like domain, while its aminoterminal domain is capable of binding to $\alpha \mathrm{v} \beta 3$ and $\alpha \mathrm{v} \beta 5$ integrins $[227,251]$. Thereby, MFGE8 can promote exosome internalization by integrin receptor expressing macrophages. In our studies preincubation with recombinant MFGE8 indeed led to an increase of exosome internalization by microglia in vitro. Nevertheless, our experiments with integrin antagonizing peptide (RGD) clearly demonstrated, that $\alpha \mathrm{v} \beta 3$ and $\alpha \mathrm{v} \beta 5$ integrins were not involved in exosome clearance. This finding is consistent with studies on MFGE8 deficient exosomes captured by DCs. Since DCs hardly express the integrin receptor in vitro $[47,251]$, it is likely that also 
alternative mechanisms account for vesicle capture.

However, the recognition of PS is not restricted to bridging molecules like MFGE8. Among the identified receptors of PS are brain angiogenesis inhibitor 1 (BAI1) [252], calreticulin [253] as well as T cell immunoglobulin domain and mucin domain 4 (TIM4) [226]. It would be interesting to test whether the direct interaction of phosphatidylserine with PS-receptors is involved in exosome clearance by microglia. Especially, since TIM-4 was recently identified as receptor for internalization of tumor-derived exosomes by macrophages [203].

\subsubsection{Oligodendroglial exosomes are cleared by macropinocytosis}

Although exosomes and myelin debris are consistent of similar lipids and proteins, the internalization mechanisms seem to be different. Myelin debris phagocytosis is particle-driven and depends on the tight interaction of different ligands with various receptors [228]. On the contrary, exosome internalization does not necessarily depend on a tight receptor interaction, as supported by our findings that oligodendroglial exosome do not tether onto microglia at low temperature. This could be explained by either low surface expression of the PS-receptor on the phagocyte or low affinity between PS and the respective PS-receptor. Nevertheless, the active role of phosphatidylserine in exosome and target cell interactions is vital and is further strengthened by recent findings, implicating PS in the internalization of T cell-derived exosomes in human monocytes [254]. Hoffmann and colleagues demonstrated that phosphatidylserine induced macropinocytosis in macrophages promoted clearance of apoptotic cells [180]. In their experiments, engulfment of apoptotic cells is a two step event: an initial tethering event mediated by phagocytic receptors, followed by PS-stimulated macropinocytosis. Similarly to our findings, the exposure of PS alone resulted in a limited adhesion to the phagocyte surface. Furthermore, it was shown that even the presence of PS alone or antibodies against the PS-receptor stimulated macropinocytosis in macrophages [180]. However, in our studies exosome internalization with PS-containing liposomes did not further stimulate macropinocy- 
tosis, but decreased the amount of exosome internalization by microglia.

\subsection{Exosome internalization by microglia: an immuno- logically silent process?}

\subsubsection{Cytokine release by microglia after exosome internalization}

Phagoctoysis of myelin components by activated microglia/ macrophages is well documented in MS and EAE, however the effect of myelin phagocytosis on microglia is controversial. Several in vitro studies showed that myelin phagocytosis by microglia and macrophages triggers release of pro-inflammatory cytokines and nitric oxide, suggesting that phagocytosis of myelin could enhance neuroinflammation [255-257]. In a striking contrast, anti-inflammatory effect of myelin phagocytosis was reported later [258, 259]. However, phagocytosis of myelin by microglia is usually associated with a highly ordered profile of cytokines and chemokines secretion that may call macrophages, neutrophils and Th2 cells into place. In vitro studies on macrophages described the release of IL-10, IL-6, CCL22 and CXCL1, which were immediately but transiently induced, while CCL2, CCL11 and TGF $\beta$ showed more persistent levels [207].

As the internalization of myelin by microglia induces the release of immunemodulatory molecules, we were interested in investigating, whether similar components packed in another "shuttle" would influence the "surveying status" of the unchallenged microglial cell. Our studies revealed that exosomes induce a slight release of MIP1 $\alpha /$ CCL3 and RANTES/CCL5 by microglia in vitro. The chemokines MIP1 $\alpha$ / CCL3 and RANTES/CCL5 are known to activate and induce chemotaxis of T cells and monocytes, which are recruited to the site of chemokine release [260]. However, the data showed that in comparison with the release induced by LPS, the impact of exosomes on the microglial cytokine release profile is rather small.

Chemokines are also involved in other functions besides chemoattraction, namely neuronal development, modulation of cell adhesion, phagocytosis or T cell activation $[138,261]$. Among the transiently expressed chemokines in the human embryonic 
brain are IL-8/CXCL8, MCP-1/CCL2, MIP1 $\alpha$ /CCL3 and RANTES/CCL5. In the developing cortex, a correlation between microglial cell colonization and the temporal and spatial pattern of expression of MCP-1/CCL2 and RANTES/CCL5 has been described, suggesting that these chemokines may take part in the migration of microglial precursors [262].

Furthermore, we observed that the internalization of oligodendroglial exosomes did not induce the release of potent pro-inflammatory inflammatory cytokine $\mathrm{TNF} \alpha$ nor IL12p40. The microglial release of $\mathrm{TNF} \alpha$ acts in a autokrine fashion by inducing its own expression in addition to other pro-inflammatory cytokines such as IL-1 $\beta$ [263]. $\mathrm{TNF} \alpha$ exhibits a direct cytotoxic effect upon oligodendrocytes and myelin in vitro [139].

In addition, microglia did not release IL-10 after ingestion of exosomes. The IL10 is a potent anti-inflammatory cytokine that functions by inhibiting the expression of pro-inflammatory cytokines such as $\mathrm{TNF} \alpha$ and $\mathrm{IFN} \gamma$ and the expression of MHCII, B7 and CD40 [264]. Taken together, this data suggests that oligodendroglial exosomes are internalized without inducing a prominent pro-inflammatory or anti-inflammatory response.

\subsection{Is exosomal antigen presented by microglia?}

Exosome internalization by microglia did not dramatically change the cytokine release profile of microglia. However, as antigen presenting cells, microglia have the potential to present putative exosome-derived antigen to effector cells of the adaptive immune system. Our experiments revealed that internalization of exosomes alone did not upregulate MHCII expression, while IFN $\gamma$ stimulation resulted in a robust increase of MHCII molecules at the cell surface of microglia.

\section{Cellular responses to IFN $\gamma$}

Our experiments confirmed the IFN $\gamma$ mediated stimulation of MHCII expression by microglia both in vitro and in vivo $[265,266]$. We could also reproduce findings showing that IFN $\gamma$ upregulates both T cell co-stimulatory ligands B7-1 and B7-2 $[267,268]$. Interestingly, the stimulation of T cells through the two B7 isoforms may 
have differential effects, with B7-1 favoring T helper cell 1 (Th1) and B7-2 favoring T helper cell 2 (Th2) responses of T cells [269, 270].

The main cellular producers of IFN $\gamma$ are activated natural killer (NK) cells [271], activated Th1 cells [272] and activated $\mathrm{CD}^{+}$cytotoxic $\mathrm{T}$ cells [273]. The release of IFN $\gamma$ shifts the microglial cytokine release from anti-inflammatory to proinflammatory cytokine profiles [274]. Moreover, IFN $\gamma$ can convert the cytokine release profile of LPS challenged microglia from chemoattraction for neutrophils to a preferential attraction of monocytes and T lymphocytes [88].

In $\mathrm{EAE}, \mathrm{IFN} \gamma$ is thought to play an important role in engaging the innate immune system into an inflammatory cascade induced by autoreactive $\mathrm{T}$ cells. During the priming phase of EAE autoreactive T cells, which recognize specific myelin autoantigens, are generated by peripheral immunization with myelin proteins or peptides [139]. In vitro studies revealed that microglia stimulated with $\mathrm{IFN} \gamma$ were capable of presenting myelin basic protein (MBP) to MBP-specific T cell lines [275]. In EAE, the autoreactive Th1 cells encounter endogenous myelin antigen presented on DCs or perivascular/ meningeal macrophages, leading to an activation of both $\mathrm{T}$ cells and APCs. In response to this interaction, T cells secrete IFN $\gamma$ which in turn acts on microglia by upregulation of MHCII, B7 and CD40 molecules and thereby enabling efficient antigen presentation. This results in a positive feedback loop [139].

Despite the extraordinary complexity of the IFN $\gamma$ response, its role can be limited to the regulation of the immune system and the control of infectious diseases. The phenotype of mutant mice lacking genes encoding IFN $\gamma$ itself, the $\alpha$-chain of the IFN $\gamma$ receptor or for the main transcription factor STAT1, revealed only alterations in the lymphoid system and increase in susceptibility to multiple infectious pathogens [276-279].

\subsubsection{Macropinocytosis and antigen presentation by microglia}

Previous studies on DCs revealed that dendritic cell derived-exosomes were efficiently internalized by DC, first sorted into recycling endosomes and finally to the late endosomes/lysosomes, where exosomal proteins were processed and loaded onto MHCII molecules. By this mechanism, DCs were capable to present peptides derived 
from the internalized exosomes to T cells [201]. To study the capacity of microglia to present exosome-derived antigen, we took advantage of a $\mathrm{T}$ cell line recognizing MOG peptides loaded onto MHCII molecules on APCs. However, our experiments show that MOG provided via MOG-positive exosomes was not presented on MHCII, while recombinant MOG was efficiently inducing a $\mathrm{T}$ cell activation.

\section{Processing of exosomal antigens for presentation on MHC molecules?}

In many APCs, MHCII molecules are clustered in MCHII enriched compartments, which contain lysosomal marker molecules such as Lamp-1 and CD63 [280, 281]. In this late endosomal compartments, in which also oligodendroglial exosomes are found after internalization by microglia (see Figure 3.5), MHCII molecules are loaded with antigenic peptides [120]. In DCs, antigens internalized by macropinocytosis are also observed in endocytic compartments enriched for MHCII [186]. After loading onto MHCII complexes, antigens are transported to the cell surface for presentation to $\mathrm{CD}^{+} \mathrm{T}$ cells. One explanation for the lack of exosome-derived MOG presentation via MHCII could be the insufficient concentration of antigenic MOG. Alternatively, exosomal antigens could also be (cross)presented via MHCI, since restricted loading of extracellular antigens is localized to the same compartment [120]. Interestingly, the route of antigen-presentation might be dependent on the way of cargo internalization. Experiments on macrophages revealed that antigen uptake by macropinocytosis produced presentation of exogenous antigen via MHCI whereas receptor-mediated endocytosis led to exclusive presentation via MHCII molecules [282].

The antigen presenting pathway by which exogenous antigen is loaded onto MHCI molecules is called "cross-presentation". Recent studies revealed that microglia can cross-present exogenous antigens to $\mathrm{CD} 8^{+} \mathrm{T}$ cells. This capacity is dependent on proteasome activity and the transfer of peptides to the ER by specialized peptide transporters (TAP) [121]. After intracerebral injection of antigen, microglia internalized and processed exogenous antigen onto MHCI in vivo and efficiently stimulated $\mathrm{CD}^{+} \mathrm{T}$ cells in vitro [283]. Further studies have demonstrated that active alkalization of the phagosome is required for cross-presentation [120]. While the macropinosome must acquire proteolytic function to process antigens, peptides must 
be spared from complete proteolytic destruction for presentation by MHC molecules, and this is achieved by regulating the level of proteolysis and phagosomal acidification in different APCs at different stages of APC activation or maturation [284, 285].

\section{Exosomal lipids as putative antigens for presentation by microglia?}

In addition to the classical concept of self/non-self peptide recognition by the immune system another pathway of antigen presentation might be applied after exosome internalization by microglia. In this pathway, CD1 molecules present lipids and glycolipids to the mammalian immune system. All members of the CD1 family share sequence homology and overall domain structure with MHCI molecules $[286,287]$ and are expressed by human antigen presenting cells, whereas murine cells just express CD1d [287].

Similarly to MHCII molecules, CD1 molecules survey the endocytic pathway for incoming exogenous antigens. The presentation of lipids requires uptake and delivery to an intracellular compartment in antigen-presenting cells [288] and is inhibited by compounds preventing the endosomal acidification [288, 289]. The acidic pH might promote lipid loading onto CD11d due to a more open structure of the binding groove [290]. Both exogenous and endogenous lipid antigens can enter the CD1 antigen processing route and be presented to CD-1 restricted T cells. A possible role of CD1-lipd presentation during inflammatory diseases affecting lipid rich tissues is highly attractive [291]. It would be interesting to determine, whether exosomal lipids could be presented via CD1 molecules.

Antigenic self-lipids stimulating group 1 CD-1 restricted T cells are phosphoglycerollipids and glycoshingolipids, such as sulfatide and sphingomyelin. Sulphatide, a major component of myelin, is presented by CD1a, CD1b and Cd1c and activates clonally restricted human T cells $[292,293]$. Further, GM1 and related gangliosides were also found to be presented by CD1b, suggesting that self-lipid antigens may be important targets for autoimmune T cells in multiple sclerosis [294, 295]. Interestingly, exosomes derived from DCs contain CD1 molecules that are capable of lipid antigen presentation [67]. 


\subsubsection{Regulation of macropinocytosis and antigen presentation by microglia}

Permanent constitutive macropinocytosis is typically restricted to phagocytic cells such as immature dendritic cells and activated macrophages [237], while macopinocytosis in cell types that do not normally phagocytose is mostly a transient response to growth factors [296-298]. We found that the macropinocytotic activity of microglia can be modified by inflammatory signals. Our experiments showed that IFN $\gamma$ stimulation of microglia results in an overall decrease in exosomes internalization, which could be also observed after stimulation of microglia with LPS. In studies accessing the macropinocytotic capacity of dendritic cells, stimulation with TNF $\alpha$ or LPS lead similarly to a downregulation of macropinocytosis [186].

Immature dendritic cells (DCs) use high levels of fluid phase uptake via macropinocytosis for antigen capture. However, they are poor APCs, because they retain most of their MHC molecules intracellularly and are unable to form peptide-MHC class II complexes [122, 299]. Upon engaging pathogen-derived products, DCs undergo maturation, which includes downregulation of macropinocytosis [186]. Inflammatory stimuli, such as LPS and TNF $\alpha$, trigger DC maturation resulting in the upregulation of surface MHCI, MHCII, CD40 and CD86. Maturation converts immature DCs adapted for antigen accumulation to cells specialized for the processing and presentation of previously encountered antigens to T cells [299, 300].

This mechanism of conversion might be also applied to microglia after the encounter of inflammatory stimuli. However, after IFN $\gamma$ stimulus we observed that only a subpopulation of microglia showed an increase of MHCII molecules, while another subtype remained MHCII negative. Our internalization experiments with IFN $\gamma$ stimulated microglia unveiled that exosomes were preferentially internalized by microglia with low levels of MHCII expression and vice versa. Internalization of putative exosomal antigen is therefore restricted to cells with less antigen presenting capacity compared to the other microglial cell pool. This subpopulation could efficiently account for antigen presentation, since recent studies have suggested that activation of macrophages with IFN $\gamma$ or LPS leads to reduced vacuolar protease activity that may avoid complete proteolitic destruction of peptides and favor generation of pep- 
tides for antigen presentation [301, 302]. Also, a full T cell activation seems to be possible since T cell co-stimulatory molecules B7-1 and B7-2 are abundant, at least in the MHCII positive microglia subpopulation (see Figure 3.27).

In summary, we concluded that the microglial population might consist of distinct subtypes with varied functions. While exosome clearance is preferentially executed by microglia with lower $\mathrm{T}$ cell stimulation capacity, putative antigen processing and presentation could assigned to other microglial subtypes.

\subsection{Functional heterogeneity of microglia}

In our study we found that microglial subpopulations might be assigned to different tasks. While one subtype was engaged in clearance, the other subpopulation showed expression of molecules necessary for peptide antigen presentation. Parenchymal microglia are described as a single homogeneous population. Microglia occur in all brain regions in an almost uniform distribution with some prevalence in hippocampus, olfactory telencephalon, basal ganglia and substantia nigra [100]. Microglia morphology, however, can vary depending on their location in brain parenchyma [100]. Further differences are found in the abundance of receptors, response to cytokines and constitutive or inducible MHC expression [303-305]. Anderson and colleagues found that Th1-regulating immune receptor TIM1 is constitutively expressed by microglia in the white matter, but absent from the grey matter [306]. Also the expression of nerve growth factor family in vivo is regionally restricted. Even more striking is that within those regions only subpopulations are actively elaborating trophins [307]. These signs of microglial heterogeneity strongly support the notion of microglial subpopulations, whereas it is not clear whether microglia are solely conditioned by their microenvironment or are additionally intrinsically programed for distinct tasks.

\subsubsection{Microglia vs. macrophages}

One major approach to define characteristics of the microglial population is the analysis of microglial gene expression [101, 233-235]. Microarray studies on non- 
activated rat microglia revealed that 326 molecules are constitutively expressed in microglia [308]. Upon IFN $\gamma$ stimulation, 34 transcripts and 7 ESTs were newly detected in the microglial transcriptome. Furthermore, IFN $\gamma$ increased the expression of 60 of 182 known molecules, while 29 of 183 genes were downregulated [308]. The upregulated genes could be primarily linked to antigen presentation, protein degradation, actin binding, cell adhesion, apoptosis and cell signaling. In contrast, molecules repressed by IFN $\gamma$ stimulation were primarily associated with growth regulation, remodeling of the extracellular matrix, lipid metabolism and lysosomal processing [308].

Using gene expression profiles microglia can be functionally and molecularly distinguished from other macrophage populations. Analysis of IFN $\gamma$ activated microglia vs. peritoneal macrophages, however, confirmed that CNS-resident microglia are heterogeneous [309]. Thus, a universal microglia-specific marker may not exist to differentiate them from infiltrating macrophages. Of the candidate genes found, C1qA is nearly expressed by all microglia throughout the microglial population, while triggering receptor expressed on myeloid cells-2 (TREM-2) and CXCL14 were only expressed in microglia subsets [309]. Microglia in regions predisposed to develop Alzheimer's disease were TREM-2 positive, microglia adjacent to the leaky blood brain barrier were TREM-2 negative. TREM-2 has been shown to induce phagocytosis in microglia and to inhibit TLR-induced cytokine production [310, 311]. Interestingly, loss-of-function mutations of TREM-2 result in the inflammatory neurodegeneration and the loss of myelin in humans [312]. Furthermore, it was described to mediate the differentiation of human monocyte-derived DCs into antigen-presenting cells [313]. Thus, microglia expressing TREM-2 may have a higher potential to differentiate into effective antigen-presenting cells and also might have a higher potential to stimulate $\mathrm{T}$ cell activation $[233,313]$. Thereby, it not only becomes clear that the microglial RNA population is as complex, as the one of peritoneal macrophages, but also that microglia display a gene expression profile distinguishable from them [233]. These findings raise the question how clearing and antigen-presenting microglia can be further characterized. One possible, but technically challenging approach would be the gene expression analysis of both subtypes. For this it would be interesting to 
compare the gene expression profiles MHCII-positive and MHCII-negative microglia after stimulation with $\operatorname{IFN} \gamma$.

\subsubsection{Microglial heterogeneity - cellular microenvironment or intrin- sic cues?}

The microglial phenotype is highly plastic and continually determined by the sum of external cues in their local microenvironment. A recent study on $\operatorname{IFN} \gamma$ stimulated cells revealed that the neonatal microglia gene expression profile was closer to that of infiltrating peripheral macrophages then to microglia isolated from the CNS [309]. This finding demonstrates the intricate complexity of the microglial response to stimulation, which is in turn affected by surrounding influences from the CNS.

The influence of a single cell type suppressing microglial activation has been shown in CD200 deficient mice. CD200 is a cell surface molecule which is expressed by neurons, while the receptor of CD200 is exclusively expressed by microglia in the CNS [314]. Mice which were lacking the expression of CD200 showed spontaneous activation of microglia in the CNS, accelerated microglial responses to injury, and clinically more severe EAE [315]. Moreover, it was shown that neuronal activity can inhibit the expression of molecules implicated in antigen-presentation, while it is induced if neuronal activity is suppressed [316]. Neuronal production of neurotrophins similarly inhibits IFN $\gamma$ induced microglial MHCII upregulation [317]. Likewise, TGF $\beta$ produced by astrocytes decreases the ability of macrophages and microglia to induce MHC II and costimulatory molecules in response to LPS or IFN $\gamma$ [318-320].

In chronic inflammatory diseases multiple "activation states" of microglia are found simultaneously [230]. Among those are the classical activation state of microglia, which is stimulated by IFN $\gamma$ and is associated with the release of pro-inflammatory cytokines [321-323] and the "alternatively activated" macrophages, mediated by IL-4 and IL-13, which induce microglial gene expression and proteins associated with the resolution of inflammation [323-325]. However, it is not yet clear whether different activation states are found on separate populations of immune-activated microglia that express only alternative or only classical activation gene profiles. It 
would be interesting to reveal if different activation states are found on regionally restricted microglia, for example in proximity to the blood vessels [230].

\subsection{Function of oligodendroglial exosomes}

We conducted this study to reveal the fate of exosomes released by oligodendrocytes. We found that exosomes are internalized by microglia, where their contents are degraded in an immunological silent manner. Putative peptide antigens were not presented via MHCII, while cross-presentation via MHCI and exposure of lipid antigens to reactive $\mathrm{T}$ cells remain to be elucidated.

Our results show that oligodendroglial exosomes can be employed as alternative pathway for degradation of material released by oligodendrocytes, as it was described for the elimination of tranferrin receptor by red blood cells [326]. In oligodendrocytes, the turn-over of myelin components could be mediated partially by the release of components to the extracellular space via exosomes or related vesicles. Apart from the endosomal origin, exosome-like vesicles can also arise by immediate budding from discrete domains of the plasma membrane that are enriched for exosomal and endosomal proteins [327].

Besides the turn-over of myelin proteins, also lipids are particularly enriched in oligodendrocytes and could be removed via the exosomal route. It was shown recently that the exosomal release of cholesterol may constitute a cellular mechanism, which bypasses the toxic lysosomal cholesterol accumulation in Niemann-Pick type C1 disease. This mechanism might be also beneficial in other lysosomal lipid storage disorders like metachromatic leukodystrophy, which is characterized by a deficiency in glycosphingolipid turn-over resulting into severe neurological disorders [328].

In comparison to the proteasomal or lysosomal degradation of proteins, the amount of cargo released via the exosomal pathway seems to be rather small. Oligodendrocytes constitutively release exosomes on a basal level. However, oligodendrocytes respond to $\mathrm{Ca}^{2+}$ levels with increased levels of exosome release in vitro [4]. The oligodendroglial $\mathrm{Ca}^{2+}$ signaling is mediated by neurotransmitters such as glutamate and ATP that act upon ligand-gated and voltage gated $\mathrm{Ca}^{2+}$ channels [329]. Hence, cytoplasmic $\mathrm{Ca}^{2+}$ levels in oligodendrocytes and exosome release could be 
coupled to neuronal activity. In addition, a recent study observed that the secretion of exosomes was dramatically reduced when cells were incubated with conditioned neuronal medium [330]. This data implies, that the release of exosomes by oligodendrocytes might underly a neuronal regulation. Interestingly, also neuronal exosome release can be stimulated. In this case the exosome secretion is regulated by potassium mediated depolarisation [31].

In addition to their implication in the cellular disposal of obsolete proteins and lipids, oligodendroglial exosomes might be part of the intercellular signaling system in the CNS. Strikingly, it was recently shown that oligodendroglial exosomes exhibit an autocrine inhibitory effect on morphological differentiation of oligodendrocytes and inhibited myelin formation in in vitro co-culture systems [330]. Importantly, exosomes are not only released by oligodendrocytes but also by neurons, astrocytes and microglial cells in vitro [31, 32, 331]. As a result of their distinct composition, each kind of central nervous system-derived exosomes may represent an attractive cellular communication system in an environment where cell motility is restricted. As part of the signaling mechanism, the immunemodulatory potential of exosomes is of high relevance. Various studies revealed that antigen-presenting cells secrete exosomes bearing antigen-presenting molecules which can function in an immunmodulatory fashion or in turn shuttle antigen to neighboring cells. Although oligodendroglial exosomes do not contain molecules implicated in the presentation of peptide antigens, they might as well have a immune-relevant function due to their protein and lipid composition. Both myelin proteins and lipid shuttled via exosomes to microglia could represent a putative pathway of antigen delivery to APCs implicated in the course of multiple sclerosis.

\subsubsection{Exosomes and autoimmunity?}

Multiple sclerosis is characterized by white matter lesions evolving into plaques that contain perivascular infiltrates of mononuclear cells including lymphocytes and macrophages [332]. It is associated with disseminated demyelination, axonal loss or damage accompanied with microglial and astrocytic changes [146, 332]. Many studies suggest that the failure to properly clear apoptotic material could ultimately 
lead to immunogenic responses and to autoimmunity. These findings are supported by the development of autoimmunity in MFGE8-deficient mice, which show impaired phagocytic capacity of macrophages [333]. However, studies on transgenic mice lacking prominent phagocytic receptors such as CD14, CD36, $\beta 3$ integrin and $\beta 5$ integrin revealed that the accumulation of apoptotic bodies does not necessarily result in autoimmunity [334, 335].

Multiple sclerosis has long been associated with viral infections. Having the ability to process antigen, microglia have the potential to participate in the course of multiple sclerosis [102]. After the initial immune response against viral antigens, macrophages and microglia phagocytosing myelin debris could participate in the presentation of the myelin protein derived epitopes, thus shifting the immune response from a viral-specific response to a myelin-specific response. Both, molecular mimicry and bystander activation are the mechanisms that initiate immunoreactivity and can lead to autoimmunity. Molecular mimicry describes the infection of viruses that share epitopes with self-determinants of myelin proteins [336] that can be presented by MHCII molecules on microglia to autoreactive CD4 ${ }^{+} \mathrm{T}$ cells [332]. In bystander activation, infection of viruses elicit strong cytokine responses that locally lead to proliferation and activation of pre-existing autoreactive $\mathrm{T}$ cells. Those $\mathrm{T}$ cells migrate to the CNS and induce CNS damage that leads finally to disease [337].

\section{Exosomes - shuttle of infectious agents and autoantigens?}

The cellular process of multi vesicular body formation and fusion with the plasma membrane provides a beneficial escape mechanism for various pathogens to avoid the host immune response. Exosomes are found to contain mycobacterial components [338] and CMV [339]. Studies have shown, that the exosome pathway is also exploited by retroviruses as a cell-encoded intercellular transport mechanism for infection [340]. HIV utilizes MVBs as the major site for accumulation in human macrophages [341] and hijacks this pathway for its budding at the cell surface [327, 342]. Thereby, exosomes represent an endogenous pathway that supports viral infection of oligodendrocytes and microglia respectively.

Our study confirmed that oligodendroglial exosomes act as a putative shuttle for 
autoantigens from oligodendrocytes to microglia, which could be - under not yet defined circumstances - be presented to autoreactive $\mathrm{T}$ cells. In multiple sclerosis, a broad spectrum of myelin proteins can be considered possible targets of autoreactive T cells. Besides MBP, also PLP and MOG have been identified [208, 343]. In addition, also myelin lipids represent potential autoantigens [295].

In summary, it is possible that exosomes containing viral or bacterial proteins are directed to microglia and exert pro-inflammatory properties that could result in the activation of the antigen-presenting cell. In this respect, self-antigens might trigger an autoimmune response. To what extend viral components are incorporated into oligodendroglial exosomes and how microliga respond to particles containing both pathogen molecules and putative autoantigens needs to be elucidated in the future. 


\section{Chapter 5}

\section{Summary and conclusions}

The transfer of antigens from oligodendrocytes to immune cells has been implicated in the pathogenesis of autoimmune diseases. How encephalitogenic antigens are transported to an immunogenic environment remains unanswered, but one attractive possibility is that membrane vesicles play a role in the transfer. Oligodendrocytes produce vast amounts of membrane during myelination. In addition, membrane is released into the extracellular space as small vesicles. Among them are exosomes of endosomal origin with a size of 50 to $100 \mathrm{~nm}$, which are shown to contain major myelin proteins and lipids, both putative autoantigens in multiple sclerosis.

In this study, we analyzed the extracellular fate of oligodendroglial-derived exosomes and addressed the question of how exosomes are transferred from the donor to the target cell. We used exosomes purified from both mouse oligodendroglial cell line and primary oligodendrocyte cultures and studied their fate employing mixed primary glial and neuronal cultures as well as in situ analysis in transgenic mice.

Our studies revealed that exosomes are specifically internalized by microglia, both in vitro and in vivo. Using pharmacological inhibitors of cellular proteins, we characterized the internalization of oligodendroglial exosomes as macropincytosis, which was independent of phagocytic receptors. The uptake of exosomes was influenced by phosphatidylserine, a lipid exposed at the outer leaflet of the exosomal membrane. Interestingly, no inflammatory or anti-inflammatory microglial response was initiated after they encounter exosomes. Only a slight release of chemokines MIP1 $\alpha$ and RANTES was detected. After challenging microglia with IFN $\gamma$, recombinant MOG was readily presented by microglia and induced a robust IL-2 release, however, we 
were not able to detect a $\mathrm{T}$ cell response to microglia that ingested myelin oligodendrocyte glycoprotein (MOG)- positive exosomes. Interestingly, we observed that not all IFN $\gamma$ stimulated microglia necessarily become antigen-presenting cells. Both MHCII-positive and MHCII-negative populations were observed. Further data indicated that exosomes were predominantly ingested by MHCII-negative microglia, while microglia expressing MHCII did not internalize exosomes.

Taken together, this study demonstrates that the macropinocytotic clearance of exosomes is an important mechanism through which microglia participate in the degradation of oligodendroglial membrane, without calling the adaptive immune system into place. However, exosome clearance is restricted to functional microglial subtypes. We propose that the constitutive macropinocytotic clearance of exosomes by a subset of microglia represents an important mechanism through which microglia participate in the degradation of oligodendroglial membrane in an immunologically "silent" manner. By designating the capacity for macropinocytosis and antigen presentation to distinct cells, degradation and immune function might be assigned to different subtypes of microglia. Future studies will have to clarify whether this mechanism prevents the presentation of autoantigens by microglia and whether this mechanism is due to the microglial local environment or to intrinsic cues. 


\section{Bibliography}

[1] U. K. Hanisch. Microglia as a source and target of cytokines. Glia, 40(2):140-55, 2002.

[2] B. T. Pan, K. Teng, C. Wu, M. Adam, and R. M. Johnstone. Electron microscopic evidence for externalization of the transferrin receptor in vesicular form in sheep reticulocytes. J Cell Biol, 101(3):942-8, 1985.

[3] B. T. Pan, R. Blostein, and R. M. Johnstone. Loss of the transferrin receptor during the maturation of sheep reticulocytes in vitro. an immunological approach. Biochem $J, 210(1): 37-47,1983$.

[4] Eva-Maria Kramer-Albers. Oligodendrocytes secrete exosomes containing major myelin and stress-protective proteins: Trophic support for axons? Proteomics Clin. Appl., 1:1446-1461, 2007.

[5] K. Trajkovic, C. Hsu, S. Chiantia, L. Rajendran, D. Wenzel, F. Wieland, P. Schwille, B. Brugger, and M. Simons. Ceramide triggers budding of exosome vesicles into multivesicular endosomes. Science, 319(5867):1244-7, 2008.

[6] C. Hsu, Y. Morohashi, S. Yoshimura, N. Manrique-Hoyos, S. Jung, M. A. Lauterbach, M. Bakhti, M. Gronborg, W. Mobius, J. Rhee, F. A. Barr, and M. Simons. Regulation of exosome secretion by rab35 and its gtpase-activating proteins tbc1d10a-c. J Cell Biol, 189(2):223-32, 2010.

[7] G. Raposo, H. W. Nijman, W. Stoorvogel, R. Liejendekker, C. V. Harding, C. J. Melief, and H. J. Geuze. B lymphocytes secrete antigen-presenting vesicles. J Exp Med, 183(3):1161-72, 1996.

[8] L. Zitvogel, A. Regnault, A. Lozier, J. Wolfers, C. Flament, D. Tenza, P. RicciardiCastagnoli, G. Raposo, and S. Amigorena. Eradication of established murine tumors using a novel cell-free vaccine: dendritic cell-derived exosomes. Nat Med, 4(5):594600, 1998. 
[9] H. F. Heijnen, A. E. Schiel, R. Fijnheer, H. J. Geuze, and J. J. Sixma. Activated platelets release two types of membrane vesicles: microvesicles by surface shedding and exosomes derived from exocytosis of multivesicular bodies and alpha-granules. Blood, 94(11):3791-9, 1999.

[10] J. M. Wilson, J. A. Whitney, and M. R. Neutra. Biogenesis of the apical endosomelysosome complex during differentiation of absorptive epithelial cells in rat ileum. $J$ Cell Sci, 100 ( Pt 1):133-43, 1991.

[11] A. M. Marzesco, P. Janich, M. Wilsch-Brauninger, V. Dubreuil, K. Langenfeld, D. Corbeil, and W. B. Huttner. Release of extracellular membrane particles carrying the stem cell marker prominin-1 (cd133) from neural progenitors and other epithelial cells. J Cell Sci, 118(Pt 13):2849-58, 2005.

[12] B. Fevrier and G. Raposo. Exosomes: endosomal-derived vesicles shipping extracellular messages. Curr Opin Cell Biol, 16(4):415-21, 2004.

[13] C. Thery, S. Amigorena, G. Raposo, and A. Clayton. Isolation and characterization of exosomes from cell culture supernatants and biological fluids. Curr Protoc Cell Biol, Chapter 3:Unit 3 22, 2006.

[14] E. Perret, A. Lakkaraju, S. Deborde, R. Schreiner, and E. Rodriguez-Boulan. Evolving endosomes: how many varieties and why? Curr Opin Cell Biol, 17(4):423-34, 2005 .

[15] J. Gruenberg. The endocytic pathway: a mosaic of domains. Nat Rev Mol Cell Biol, 2(10):721-30, 2001.

[16] S. Mukherjee, T. T. Soe, and F. R. Maxfield. Endocytic sorting of lipid analogues differing solely in the chemistry of their hydrophobic tails. J Cell Biol, 144(6):127184, 1999.

[17] K. W. Dunn, T. E. McGraw, and F. R. Maxfield. Iterative fractionation of recycling receptors from lysosomally destined ligands in an early sorting endosome. $J$ Cell Biol, 109(6 Pt 2):3303-14, 1989.

[18] S. K. Rao, C. Huynh, V. Proux-Gillardeaux, T. Galli, and N. W. Andrews. Identification of snares involved in synaptotagmin vii-regulated lysosomal exocytosis. $J$ Biol Chem, 279(19):20471-9, 2004.

[19] D. J. Katzmann, S. Sarkar, T. Chu, A. Audhya, and S. D. Emr. Multivesicular body sorting: ubiquitin ligase rsp5 is required for the modification and sorting of carboxypeptidase s. Mol Biol Cell, 15(2):468-80, 2004. 
[20] G. van Niel, R. Wubbolts, T. Ten Broeke, S. I. Buschow, F. A. Ossendorp, C. J. Melief, G. Raposo, B. W. van Balkom, and W. Stoorvogel. Dendritic cells regulate exposure of mhc class ii at their plasma membrane by oligoubiquitination. Immunity, 25(6):885-94, 2006.

[21] R. C. Piper and D. J. Katzmann. Biogenesis and function of multivesicular bodies. Annu Rev Cell Dev Biol, 23:519-47, 2007.

[22] J. H. Hurley. Escrt complexes and the biogenesis of multivesicular bodies. Curr Opin Cell Biol, 20(1):4-11, 2008.

[23] R. L. Williams and S. Urbe. The emerging shape of the escrt machinery. Nat Rev Mol Cell Biol, 8(5):355-68, 2007.

[24] A. de Gassart, C. Geminard, B. Fevrier, G. Raposo, and M. Vidal. Lipid raftassociated protein sorting in exosomes. Blood, 102(13):4336-44, 2003.

[25] M. Vidal, P. Mangeat, and D. Hoekstra. Aggregation reroutes molecules from a recycling to a vesicle-mediated secretion pathway during reticulocyte maturation. $J$ Cell Sci, 110 ( Pt 16):1867-77, 1997.

[26] G. Van Niel, J. Mallegol, C. Bevilacqua, C. Candalh, S. Brugiere, E. TomaskovicCrook, J. K. Heath, N. Cerf-Bensussan, and M. Heyman. Intestinal epithelial exosomes carry mhc class ii/peptides able to inform the immune system in mice. Gut, $52(12): 1690-7,2003$

[27] N. Aoki, S. Jin-no, Y. Nakagawa, N. Asai, E. Arakawa, N. Tamura, T. Tamura, and T. Matsuda. Identification and characterization of microvesicles secreted by 3t3-11 adipocytes: redox- and hormone-dependent induction of milk fat globule-epidermal growth factor 8-associated microvesicles. Endocrinology, 148(8):3850-62, 2007.

[28] N. Chaput, C. Flament, S. Viaud, J. Taieb, S. Roux, A. Spatz, F. Andre, J. B. LePecq, M. Boussac, J. Garin, S. Amigorena, C. Thery, and L. Zitvogel. Dendritic cell derived-exosomes: biology and clinical implementations. J Leukoc Biol, 80(3):471-8, 2006.

[29] D. S. Choi, J. M. Lee, G. W. Park, H. W. Lim, J. Y. Bang, Y. K. Kim, K. H. Kwon, H. J. Kwon, K. P. Kim, and Y. S. Gho. Proteomic analysis of microvesicles derived from human colorectal cancer cells. J Proteome Res, 6(12):4646-55, 2007.

[30] J. Conde-Vancells, E. Rodriguez-Suarez, N. Embade, D. Gil, R. Matthiesen, M. Valle, F. Elortza, S. C. Lu, J. M. Mato, and J. M. Falcon-Perez. Characterization and comprehensive proteome profiling of exosomes secreted by hepatocytes. J Proteome Res, 2008. 
[31] J. Faure, G. Lachenal, M. Court, J. Hirrlinger, C. Chatellard-Causse, B. Blot, J. Grange, G. Schoehn, Y. Goldberg, V. Boyer, F. Kirchhoff, G. Raposo, J. Garin, and R. Sadoul. Exosomes are released by cultured cortical neurones. Mol Cell Neurosci, 31(4):642-8, 2006.

[32] I. Potolicchio, G. J. Carven, X. Xu, C. Stipp, R. J. Riese, L. J. Stern, and L. Santambrogio. Proteomic analysis of microglia-derived exosomes: metabolic role of the aminopeptidase cd13 in neuropeptide catabolism. J Immunol, 175(4):2237-43, 2005.

[33] R. Mears, R. A. Craven, S. Hanrahan, N. Totty, C. Upton, S. L. Young, P. Patel, P. J. Selby, and R. E. Banks. Proteomic analysis of melanoma-derived exosomes by twodimensional polyacrylamide gel electrophoresis and mass spectrometry. Proteomics, 4(12):4019-31, 2004.

[34] D. Skokos, S. Le Panse, I. Villa, J. C. Rousselle, R. Peronet, B. David, A. Namane, and S. Mecheri. Mast cell-dependent $\mathrm{b}$ and $\mathrm{t}$ lymphocyte activation is mediated by the secretion of immunologically active exosomes. J Immunol, 166(2):868-76, 2001.

[35] R. Wubbolts, R. S. Leckie, P. T. Veenhuizen, G. Schwarzmann, W. Mobius, J. Hoernschemeyer, J. W. Slot, H. J. Geuze, and W. Stoorvogel. Proteomic and biochemical analyses of human b cell-derived exosomes. potential implications for their function and multivesicular body formation. J Biol Chem, 278(13):10963-72, 2003.

[36] J. P. Hegmans, M. P. Bard, A. Hemmes, T. M. Luider, M. J. Kleijmeer, J. B. Prins, L. Zitvogel, S. A. Burgers, H. C. Hoogsteden, and B. N. Lambrecht. Proteomic analysis of exosomes secreted by human mesothelioma cells. Am J Pathol, 164(5):1807-15, 2004.

[37] M. P. Bard, J. P. Hegmans, A. Hemmes, T. M. Luider, R. Willemsen, L. A. Severijnen, J. P. van Meerbeeck, S. A. Burgers, H. C. Hoogsteden, and B. N. Lambrecht. Proteomic analysis of exosomes isolated from human malignant pleural effusions. Am J Respir Cell Mol Biol, 31(1):114-21, 2004.

[38] T. Pisitkun, R. F. Shen, and M. A. Knepper. Identification and proteomic profiling of exosomes in human urine. Proc Natl Acad Sci U S A, 101(36):13368-73, 2004.

[39] J. L. Gatti, S. Metayer, M. Belghazi, F. Dacheux, and J. L. Dacheux. Identification, proteomic profiling, and origin of ram epididymal fluid exosome-like vesicles. Biol Reprod, 72(6):1452-65, 2005.

[40] K. Laulagnier, D. Grand, A. Dujardin, S. Hamdi, H. Vincent-Schneider, D. Lankar, J. P. Salles, C. Bonnerot, B. Perret, and M. Record. Pld2 is enriched on exosomes 
and its activity is correlated to the release of exosomes. FEBS Lett, 572(1-3):11-4, 2004 .

[41] M. Vidal, J. Sainte-Marie, J. R. Philippot, and A. Bienvenue. Asymmetric distribution of phospholipids in the membrane of vesicles released during in vitro maturation of guinea pig reticulocytes: evidence precluding a role for "aminophospholipid translocase". J Cell Physiol, 140(3):455-62, 1989.

[42] C. Subra, K. Laulagnier, B. Perret, and M. Record. Exosome lipidomics unravels lipid sorting at the level of multivesicular bodies. Biochimie, 89(2):205-12, 2007.

[43] J. S. Schorey and S. Bhatnagar. Exosome function: from tumor immunology to pathogen biology. Traffic, 9(6):871-81, 2008.

[44] C. Thery, M. Boussac, P. Veron, P. Ricciardi-Castagnoli, G. Raposo, J. Garin, and S. Amigorena. Proteomic analysis of dendritic cell-derived exosomes: a secreted subcellular compartment distinct from apoptotic vesicles. J Immunol, 166(12):730918, 2001.

[45] S. Mathivanan, H. Ji, and R. J. Simpson. Exosomes: extracellular organelles important in intercellular communication. J Proteomics, 73(10):1907-20, 2010.

[46] R. Gastpar, M. Gehrmann, M. A. Bausero, A. Asea, C. Gross, J. A. Schroeder, and G. Multhoff. Heat shock protein 70 surface-positive tumor exosomes stimulate migratory and cytolytic activity of natural killer cells. Cancer Res, 65(12):5238-47, 2005.

[47] E. Segura, S. Amigorena, and C. Thery. Mature dendritic cells secrete exosomes with strong ability to induce antigen-specific effector immune responses. Blood Cells Mol Dis, 35(2):89-93, 2005.

[48] N. Blanchard, D. Lankar, F. Faure, A. Regnault, C. Dumont, G. Raposo, and C. Hivroz. Tcr activation of human $t$ cells induces the production of exosomes bearing the tcr/cd3/zeta complex. J Immunol, 168(7):3235-41, 2002.

[49] P. J. Peters, H. J. Geuze, H. A. Van der Donk, J. W. Slot, J. M. Griffith, N. J. Stam, H. C. Clevers, and J. Borst. Molecules relevant for t cell-target cell interaction are present in cytolytic granules of human t lymphocytes. Eur J Immunol, 19(8):1469$75,1989$.

[50] S. C. Saunderson, P. C. Schuberth, A. C. Dunn, L. Miller, B. D. Hock, P. A. MacKay, N. Koch, R. W. Jack, and A. D. McLellan. Induction of exosome release in primary b cells stimulated via cd40 and the il-4 receptor. J Immunol, 180(12):8146-52, 2008. 
[51] S. Bhatnagar and J. S. Schorey. Exosomes released from infected macrophages contain mycobacterium avium glycopeptidolipids and are proinflammatory. J Biol Chem, 282(35):25779-89, 2007.

[52] G. van Niel, I. Porto-Carreiro, S. Simoes, and G. Raposo. Exosomes: a common pathway for a specialized function. J Biochem, 140(1):13-21, 2006.

[53] A. Savina, M. Furlan, M. Vidal, and M. I. Colombo. Exosome release is regulated by a calcium-dependent mechanism in k562 cells. J Biol Chem, 278(22):20083-90, 2003.

[54] M. M. Menager, G. Menasche, M. Romao, P. Knapnougel, C. H. Ho, M. Garfa, G. Raposo, J. Feldmann, A. Fischer, and G. de Saint Basile. Secretory cytotoxic granule maturation and exocytosis require the effector protein hmunc13-4. Nat Immunol, 8(3):257-67, 2007.

[55] M. Ostrowski, N. B. Carmo, S. Krumeich, I. Fanget, G. Raposo, A. Savina, C. F. Moita, K. Schauer, A. N. Hume, R. P. Freitas, B. Goud, P. Benaroch, N. Hacohen, M. Fukuda, C. Desnos, M. C. Seabra, F. Darchen, S. Amigorena, L. F. Moita, and C. Thery. Rab27a and rab27b control different steps of the exosome secretion pathway. Nat Cell Biol, 12(1):19-30; sup pp 1-13.

[56] A. Savina, C. M. Fader, M. T. Damiani, and M. I. Colombo. Rab11 promotes docking and fusion of multivesicular bodies in a calcium-dependent manner. Traffic, 6(2):131-43, 2005.

[57] R. J. Loomis, D. A. Holmes, A. Elms, P. A. Solski, C. J. Der, and L. Su. Citron kinase, a rhoa effector, enhances hiv-1 virion production by modulating exocytosis. Traffic, 7(12):1643-53, 2006.

[58] A. Stoeck, S. Keller, S. Riedle, M. P. Sanderson, S. Runz, F. Le Naour, P. Gutwein, A. Ludwig, E. Rubinstein, and P. Altevogt. A role for exosomes in the constitutive and stimulus-induced ectodomain cleavage of 11 and cd44. Biochem J, 393(Pt 3):609$18,2006$.

[59] S. Keller, C. Rupp, A. Stoeck, S. Runz, M. Fogel, S. Lugert, H. D. Hager, M. S. Abdel-Bakky, P. Gutwein, and P. Altevogt. Cd24 is a marker of exosomes secreted into urine and amniotic fluid. Kidney Int, 72(9):1095-102, 2007.

[60] A. Llorente, B. van Deurs, and K. Sandvig. Cholesterol regulates prostasome release from secretory lysosomes in pc-3 human prostate cancer cells. Eur J Cell Biol, 86(7):405-15, 2007. 
[61] C. Lebrand, M. Corti, H. Goodson, P. Cosson, V. Cavalli, N. Mayran, J. Faure, and J. Gruenberg. Late endosome motility depends on lipids via the small gtpase rab7. EMBO J, 21(6):1289-300, 2002.

[62] X. Yu, S. L. Harris, and A. J. Levine. The regulation of exosome secretion: a novel function of the p53 protein. Cancer Res, 66(9):4795-801, 2006.

[63] C. Thery, A. Regnault, J. Garin, J. Wolfers, L. Zitvogel, P. Ricciardi-Castagnoli, G. Raposo, and S. Amigorena. Molecular characterization of dendritic cell-derived exosomes. selective accumulation of the heat shock protein hsc73. J Cell Biol, 147(3):599-610, 1999.

[64] J. Colino and C. M. Snapper. Exosomes from bone marrow dendritic cells pulsed with diphtheria toxoid preferentially induce type 1 antigen-specific igg responses in naive recipients in the absence of free antigen. $J$ Immunol, 177(6):3757-62, 2006.

[65] B. T. Pan and R. Johnstone. Selective externalization of the transferrin receptor by sheep reticulocytes in vitro. response to ligands and inhibitors of endocytosis. $J$ Biol Chem, 259(15):9776-82, 1984.

[66] K. Strauss, C. Goebel, H. Runz, W. Mobius, S. Weiss, I. Feussner, M. Simons, and A. Schneider. Exosome secretion ameliorates lysosomal storage of cholesterol in niemann-pick type c disease. J Biol Chem, 285(34):26279-88, 2010.

[67] H. G. Lamparski, A. Metha-Damani, J. Y. Yao, S. Patel, D. H. Hsu, C. Ruegg, and J. B. Le Pecq. Production and characterization of clinical grade exosomes derived from dendritic cells. J Immunol Methods, 270(2):211-26, 2002.

[68] J. Wolfers, A. Lozier, G. Raposo, A. Regnault, C. Thery, C. Masurier, C. Flament, S. Pouzieux, F. Faure, T. Tursz, E. Angevin, S. Amigorena, and L. Zitvogel. Tumor-derived exosomes are a source of shared tumor rejection antigens for ctl cross-priming. Nat Med, 7(3):297-303, 2001.

[69] H. Peche, M. Heslan, C. Usal, S. Amigorena, and M. C. Cuturi. Presentation of donor major histocompatibility complex antigens by bone marrow dendritic cellderived exosomes modulates allograft rejection. Transplantation, 76(10):1503-10, 2003.

[70] M. Karlsson, S. Lundin, U. Dahlgren, H. Kahu, I. Pettersson, and E. Telemo. "tolerosomes" are produced by intestinal epithelial cells. Eur J Immunol, 31(10):2892-900, 2001. 
[71] J. Mallegol, G. Van Niel, C. Lebreton, Y. Lepelletier, C. Candalh, C. Dugave, J. K. Heath, G. Raposo, N. Cerf-Bensussan, and M. Heyman. T84-intestinal epithelial exosomes bear mhc class ii/peptide complexes potentiating antigen presentation by dendritic cells. Gastroenterology, 132(5):1866-76, 2007.

[72] H. Valadi, K. Ekstrom, A. Bossios, M. Sjostrand, J. J. Lee, and J. O. Lotvall. Exosome-mediated transfer of mrnas and micrornas is a novel mechanism of genetic exchange between cells. Nat Cell Biol, 9(6):654-9, 2007.

[73] M. Guescini, S. Genedani, V. Stocchi, and L. F. Agnati. Astrocytes and glioblastoma cells release exosomes carrying mtdna. J Neural Transm, 117(1):1-4.

[74] C. Admyre, J. Grunewald, J. Thyberg, S. Gripenback, G. Tornling, A. Eklund, A. Scheynius, and S. Gabrielsson. Exosomes with major histocompatibility complex class ii and co-stimulatory molecules are present in human bal fluid. Eur Respir J, 22(4):578-83, 2003.

[75] M. P. Caby, D. Lankar, C. Vincendeau-Scherrer, G. Raposo, and C. Bonnerot. Exosomal-like vesicles are present in human blood plasma. Int Immunol, 17(7):87987, 2005.

[76] K. Denzer, M. van Eijk, M. J. Kleijmeer, E. Jakobson, C. de Groot, and H. J. Geuze. Follicular dendritic cells carry mhc class ii-expressing microvesicles at their surface. J Immunol, 165(3):1259-65, 2000.

[77] D. Gray, M. Kosco, and B. Stockinger. Novel pathways of antigen presentation for the maintenance of memory. Int Immunol, 3(2):141-8, 1991.

[78] D. D. Taylor and C. Gercel-Taylor. Microrna signatures of tumor-derived exosomes as diagnostic biomarkers of ovarian cancer. Gynecol Oncol, 110(1):13-21, 2008.

[79] G. Rabinowits, C. Gercel-Taylor, J. M. Day, D. D. Taylor, and G. H. Kloecker. Exosomal microrna: a diagnostic marker for lung cancer. Clin Lung Cancer, 10(1):42-6, 2009 .

[80] J. Nilsson, J. Skog, A. Nordstrand, V. Baranov, L. Mincheva-Nilsson, X. O. Breakefield, and A. Widmark. Prostate cancer-derived urine exosomes: a novel approach to biomarkers for prostate cancer. Br J Cancer, 100(10):1603-7, 2009.

[81] P. A. Gonzales, T. Pisitkun, J. D. Hoffert, D. Tchapyjnikov, R. A. Star, R. Kleta, N. S. Wang, and M. A. Knepper. Large-scale proteomics and phosphoproteomics of urinary exosomes. J Am Soc Nephrol, 20(2):363-79, 2009. 
[82] K. A. Nave. Myelination and the trophic support of long axons. Nat Rev Neurosci, 11(4):275-83, 2010.

[83] S. E. Pfeiffer, A. E. Warrington, and R. Bansal. The oligodendrocyte and its many cellular processes. Trends Cell Biol, 3(6):191-7, 1993.

[84] B. A. Barres. The mystery and magic of glia: a perspective on their roles in health and disease. Neuron, 60(3):430-40, 2008.

[85] R. D. Fields and B. Stevens-Graham. New insights into neuron-glia communication. Science, 298(5593):556-62, 2002.

[86] W. J. Streit, R. E. Mrak, and W. S. Griffin. Microglia and neuroinflammation: a pathological perspective. J Neuroinflammation, 1(1):14, 2004.

[87] B. Becher, B. G. Durell, A. V. Miga, W. F. Hickey, and R. J. Noelle. The clinical course of experimental autoimmune encephalomyelitis and inflammation is controlled by the expression of cd40 within the central nervous system. J Exp Med, 193(8):967-74, 2001.

[88] K. G. Hausler, M. Prinz, C. Nolte, J. R. Weber, R. R. Schumann, H. Kettenmann, and U. K. Hanisch. Interferon-gamma differentially modulates the release of cytokines and chemokines in lipopolysaccharide- and pneumococcal cell wallstimulated mouse microglia and macrophages. Eur J Neurosci, 16(11):2113-22, 2002 .

[89] W. Baron and D. Hoekstra. On the biogenesis of myelin membranes: sorting, trafficking and cell polarity. FEBS Lett, 584(9):1760-70, 2010.

[90] H. B. Werner, K. Kuhlmann, S. Shen, M. Uecker, A. Schardt, K. Dimova, F. Orfaniotou, A. Dhaunchak, B. G. Brinkmann, W. Mobius, L. Guarente, P. CasacciaBonnefil, O. Jahn, and K. A. Nave. Proteolipid protein is required for transport of sirtuin 2 into cns myelin. J Neurosci, 27(29):7717-30, 2007.

[91] B. D. Trapp, L. Bernier, S. B. Andrews, and D. R. Colman. Cellular and subcellular distribution of 2',3'-cyclic nucleotide 3'-phosphodiesterase and its mrna in the rat central nervous system. J Neurochem, 51(3):859-68, 1988.

[92] M. V. Gardinier, P. Amiguet, C. Linington, and J. M. Matthieu. Myelin/oligodendrocyte glycoprotein is a unique member of the immunoglobulin superfamily. J Neurosci Res, 33(1):177-87, 1992.

[93] J. N. Larocca and A. G. Rodriguez-Gabin. Myelin biogenesis: vesicle transport in oligodendrocytes. Neurochem Res, 27(11):1313-29, 2002. 
[94] A. E. Warrington and S. E. Pfeiffer. Proliferation and differentiation of o4+ oligodendrocytes in postnatal rat cerebellum: analysis in unfixed tissue slices using antiglycolipid antibodies. J Neurosci Res, 33(2):338-53, 1992.

[95] M. C. Raff. Glial cell diversification in the rat optic nerve. Science, 243(4897):1450$5,1989$.

[96] R. Bansal, K. Stefansson, and S. E. Pfeiffer. Proligodendroblast antigen (poa), a developmental antigen expressed by a007/o4-positive oligodendrocyte progenitors prior to the appearance of sulfatide and galactocerebroside. J Neurochem, 58(6):2221-9, 1992.

[97] F. M. Goni and A. Alonso. Biophysics of sphingolipids i. membrane properties of sphingosine, ceramides and other simple sphingolipids. Biochim Biophys Acta, 1758(12):1902-21, 2006.

[98] R. B. Banati. Neuropathological imaging: in vivo detection of glial activation as a measure of disease and adaptive change in the brain. Br Med Bull, 65:121-31, 2003.

[99] J. R. Chan. Myelination: all about rac 'n' roll. J Cell Biol, 177(6):953-5, 2007.

[100] L. J. Lawson, V. H. Perry, P. Dri, and S. Gordon. Heterogeneity in the distribution and morphology of microglia in the normal adult mouse brain. Neuroscience, 39(1):151-70, 1990.

[101] M. J. Carson, J. C. Thrash, and D. Lo. Analysis of microglial gene expression: identifying targets for cns neurodegenerative and autoimmune disease. Am J Pharmacogenomics, 4(5):321-30, 2004.

[102] M. J. Carson. Microglia as liaisons between the immune and central nervous systems: functional implications for multiple sclerosis. Glia, 40(2):218-31, 2002.

[103] D. Davalos, J. Grutzendler, G. Yang, J. V. Kim, Y. Zuo, S. Jung, D. R. Littman, M. L. Dustin, and W. B. Gan. Atp mediates rapid microglial response to local brain injury in vivo. Nat Neurosci, 8(6):752-8, 2005.

[104] H. Wake, A. J. Moorhouse, S. Jinno, S. Kohsaka, and J. Nabekura. Resting microglia directly monitor the functional state of synapses in vivo and determine the fate of ischemic terminals. J Neurosci, 29(13):3974-80, 2009.

[105] W. F. Hickey and H. Kimura. Perivascular microglial cells of the cns are bone marrow-derived and present antigen in vivo. Science, 239(4837):290-2, 1988. 
[106] Y. Matsumoto and M. Fujiwara. Absence of donor-type major histocompatibility complex class i antigen-bearing microglia in the rat central nervous system of radiation bone marrow chimeras. J Neuroimmunol, 17(1):71-82, 1987.

[107] L. Vallieres and P. E. Sawchenko. Bone marrow-derived cells that populate the adult mouse brain preserve their hematopoietic identity. J Neurosci, 23(12):5197$207,2003$.

[108] U. K. Hanisch and H. Kettenmann. Microglia: active sensor and versatile effector cells in the normal and pathologic brain. Nat Neurosci, 10(11):1387-94, 2007.

[109] I. Napoli, K. Kierdorf, and H. Neumann. Microglial precursors derived from mouse embryonic stem cells. Glia, 57(15):1660-71, 2009.

[110] G. W. Kreutzberg. Microglia: a sensor for pathological events in the cns. Trends Neurosci, 19(8):312-8, 1996.

[111] A. Nimmerjahn, F. Kirchhoff, and F. Helmchen. Resting microglial cells are highly dynamic surveillants of brain parenchyma in vivo. Science, 308(5726):1314-8, 2005.

[112] F. L. Heppner, M. Greter, D. Marino, J. Falsig, G. Raivich, N. Hovelmeyer, A. Waisman, T. Rulicke, M. Prinz, J. Priller, B. Becher, and A. Aguzzi. Experimental autoimmune encephalomyelitis repressed by microglial paralysis. Nat Med, 11(2):146$52,2005$.

[113] W. F. Hickey, B. L. Hsu, and H. Kimura. T-lymphocyte entry into the central nervous system. J Neurosci Res, 28(2):254-60, 1991.

[114] Jr. Janeway, C. A. and R. Medzhitov. Innate immune recognition. Annu Rev Immunol, 20:197-216, 2002.

[115] I. Glezer, A. R. Simard, and S. Rivest. Neuroprotective role of the innate immune system by microglia. Neuroscience, 147(4):867-83, 2007.

[116] S. Akira, S. Uematsu, and O. Takeuchi. Pathogen recognition and innate immunity. Cell, 124(4):783-801, 2006.

[117] C. A. Colton. Heterogeneity of microglial activation in the innate immune response in the brain. J Neuroimmune Pharmacol, 4(4):399-418, 2009.

[118] G. Zhang and S. Ghosh. Toll-like receptor-mediated nf-kappab activation: a phylogenetically conserved paradigm in innate immunity. J Clin Invest, 107(1):13-9, 2001. 
[119] S. L. Bailey, P. A. Carpentier, E. J. McMahon, W. S. Begolka, and S. D. Miller. Innate and adaptive immune responses of the central nervous system. Crit Rev Immunol, 26(2):149-88, 2006.

[120] A. Savina and S. Amigorena. Phagocytosis and antigen presentation in dendritic cells. Immunol Rev, 219:143-56, 2007.

[121] P. Guermonprez, J. Valladeau, L. Zitvogel, C. Thery, and S. Amigorena. Antigen presentation and t cell stimulation by dendritic cells. Annu Rev Immunol, 20:621-67, 2002 .

[122] P. Pierre, S. J. Turley, E. Gatti, M. Hull, J. Meltzer, A. Mirza, K. Inaba, R. M. Steinman, and I. Mellman. Developmental regulation of mhc class ii transport in mouse dendritic cells. Nature, 388(6644):787-92, 1997.

[123] M. Raghavan, N. Del Cid, S. M. Rizvi, and L. R. Peters. Mhc class i assembly: out and about. Trends Immunol, 29(9):436-43, 2008.

[124] P. Cresswell. Antigen presentation. getting peptides into mhc class ii molecules. Curr Biol, 4(6):541-3, 1994.

[125] P. R. Wolf and H. L. Ploegh. How mhc class ii molecules acquire peptide cargo: biosynthesis and trafficking through the endocytic pathway. Annu Rev Cell Dev Biol, 11:267-306, 1995.

[126] J. J. Neefjes, V. Stollorz, P. J. Peters, H. J. Geuze, and H. L. Ploegh. The biosynthetic pathway of mhc class ii but not class i molecules intersects the endocytic route. Cell, 61(1):171-83, 1990.

[127] P. Benaroch, M. Yilla, G. Raposo, K. Ito, K. Miwa, H. J. Geuze, and H. L. Ploegh. How mhc class ii molecules reach the endocytic pathway. EMBO J, 14(1):37-49, 1995.

[128] K. Williams, X. Alvarez, and A. A. Lackner. Central nervous system perivascular cells are immunoregulatory cells that connect the cns with the peripheral immune system. Glia, 36(2):156-64, 2001.

[129] F. Aloisi. Immune function of microglia. Glia, 36(2):165-79, 2001.

[130] P. Rezaie, K. Patel, and D. K. Male. Microglia in the human fetal spinal cordpatterns of distribution, morphology and phenotype. Brain Res Dev Brain Res, 115(1):71-81, 1999. 
[131] J. K. Olson, A. M. Girvin, and S. D. Miller. Direct activation of innate and antigenpresenting functions of microglia following infection with theiler's virus. $J$ Virol, $75(20): 9780-9,2001$.

[132] L. T. Nguyen, S. Radhakrishnan, B. Ciric, K. Tamada, T. Shin, D. M. Pardoll, L. Chen, M. Rodriguez, and L. R. Pease. Cross-linking the b7 family molecule b7-dc directly activates immune functions of dendritic cells. J Exp Med, 196(10):1393-8, 2002 .

[133] R. M. Ransohoff and V. H. Perry. Microglial physiology: unique stimuli, specialized responses. Annu Rev Immunol, 27:119-45, 2009.

[134] H. Lassmann, F. Zimprich, K. Vass, and W. F. Hickey. Microglial cells are a component of the perivascular glia limitans. J Neurosci Res, 28(2):236-43, 1991.

[135] M. B. Graeber, W. J. Streit, D. Buringer, D. L. Sparks, and G. W. Kreutzberg. Ultrastructural location of major histocompatibility complex (mhc) class ii positive perivascular cells in histologically normal human brain. J Neuropathol Exp Neurol, 51(3):303-11, 1992.

[136] J. Bauer, H. Rauschka, and H. Lassmann. Inflammation in the nervous system: the human perspective. Glia, 36(2):235-43, 2001.

[137] T. R. Mosmann and S. Sad. The expanding universe of t-cell subsets: Th1, th2 and more. Immunol Today, 17(3):138-46, 1996.

[138] L. Cartier, O. Hartley, M. Dubois-Dauphin, and K. H. Krause. Chemokine receptors in the central nervous system: role in brain inflammation and neurodegenerative diseases. Brain Res Brain Res Rev, 48(1):16-42, 2005.

[139] T. D. Merson, M. D. Binder, and T. J. Kilpatrick. Role of cytokines as mediators and regulators of microglial activity in inflammatory demyelination of the cns. Neuromolecular Med, 12(2):99-132.

[140] T. Schmitz and L. J. Chew. Cytokines and myelination in the central nervous system. Scientific WorldJournal, 8:1119-47, 2008.

[141] U. V. Malipiero, K. Frei, and A. Fontana. Production of hemopoietic colonystimulating factors by astrocytes. J Immunol, 144(10):3816-21, 1990.

[142] D. Giulian, B. Johnson, J. F. Krebs, J. K. George, and M. Tapscott. Microglial mitogens are produced in the developing and injured mammalian brain. $J$ Cell Biol, 112(2):323-33, 1991. 
[143] A. Takeuchi, O. Miyaishi, K. Kiuchi, and K. Isobe. Macrophage colony-stimulating factor is expressed in neuron and microglia after focal brain injury. J Neurosci Res, 65(1):38-44, 2001.

[144] H. L. Weiner. Multiple sclerosis is an inflammatory t-cell-mediated autoimmune disease. Arch Neurol, 61(10):1613-5, 2004.

[145] D. E. McFarlin and H. F. McFarland. Multiple sclerosis (first of two parts). $N$ Engl J Med, 307(19):1183-8, 1982.

[146] M. Sospedra and R. Martin. Immunology of multiple sclerosis. Annu Rev Immunol, 23:683-747, 2005.

[147] J. Hillert and O. Olerup. Multiple sclerosis is associated with genes within or close to the hla-dr-dq subregion on a normal dr15,dq6,dw2 haplotype. Neurology, 43(1):1638, 1993.

[148] J. L. Haines, H. A. Terwedow, K. Burgess, M. A. Pericak-Vance, J. B. Rimmler, E. R. Martin, J. R. Oksenberg, R. Lincoln, D. Y. Zhang, D. R. Banatao, N. Gatto, D. E. Goodkin, and S. L. Hauser. Linkage of the mhe to familial multiple sclerosis suggests genetic heterogeneity. the multiple sclerosis genetics group. Hum Mol Genet, 7(8):1229-34, 1998.

[149] E. N. Benveniste. Cytokines: influence on glial cell gene expression and function. Chem Immunol, 69:31-75, 1997.

[150] B. Becher, B. G. Durell, and R. J. Noelle. Il-23 produced by cns-resident cells controls t cell encephalitogenicity during the effector phase of experimental autoimmune encephalomyelitis. J Clin Invest, 112(8):1186-91, 2003.

[151] I. Napoli and H. Neumann. Microglial clearance function in health and disease. Neuroscience, 158(3):1030-8, 2009.

[152] I. Napoli and H. Neumann. Protective effects of microglia in multiple sclerosis. Exp Neurol, 225(1):24-8, 2009.

[153] R. N. Aravalli, P. K. Peterson, and J. R. Lokensgard. Toll-like receptors in defense and damage of the central nervous system. J Neuroimmune Pharmacol, 2(4):297$312,2007$.

[154] G. J. Doherty and H. T. McMahon. Mechanisms of endocytosis. Annu Rev Biochem, 78:857-902, 2009. 
[155] J. Savill. Recognition and phagocytosis of cells undergoing apoptosis. Br Med Bull, 53(3):491-508, 1997.

[156] N. Platt, R. P. da Silva, and S. Gordon. Recognizing death: the phagocytosis of apoptotic cells. Trends Cell Biol, 8(9):365-72, 1998.

[157] M. E. Smith. Phagocytic properties of microglia in vitro: implications for a role in multiple sclerosis and eae. Microsc Res Tech, 54(2):81-94, 2001.

[158] J. Savill, N. Hogg, Y. Ren, and C. Haslett. Thrombospondin cooperates with cd36 and the vitronectin receptor in macrophage recognition of neutrophils undergoing apoptosis. J Clin Invest, 90(4):1513-22, 1992.

[159] A. Devitt, O. D. Moffatt, C. Raykundalia, J. D. Capra, D. L. Simmons, and C. D. Gregory. Human cd14 mediates recognition and phagocytosis of apoptotic cells. Nature, 392(6675):505-9, 1998.

[160] A. M. Pearson. Scavenger receptors in innate immunity. Curr Opin Immunol, 8(1):20-8, 1996.

[161] A. Rigotti, S. L. Acton, and M. Krieger. The class b scavenger receptors sr-bi and cd36 are receptors for anionic phospholipids. J Biol Chem, 270(27):16221-4, 1995.

[162] J. Koenigsknecht and G. Landreth. Microglial phagocytosis of fibrillar beta-amyloid through a beta1 integrin-dependent mechanism. J Neurosci, 24(44):9838-46, 2004.

[163] G. Kaplan. Differences in the mode of phagocytosis with fc and c3 receptors in macrophages. Scand J Immunol, 6(8):797-807, 1977.

[164] E. Caron and A. Hall. Identification of two distinct mechanisms of phagocytosis controlled by different rho gtpases. Science, 282(5394):1717-21, 1998.

[165] M. C. Carroll. The role of complement and complement receptors in induction and regulation of immunity. Annu Rev Immunol, 16:545-68, 1998.

[166] S. L. Newman, L. K. Mikus, and M. A. Tucci. Differential requirements for cellular cytoskeleton in human macrophage complement receptor- and fc receptor-mediated phagocytosis. J Immunol, 146(3):967-74, 1991.

[167] A. Aderem and D. M. Underhill. Mechanisms of phagocytosis in macrophages. Annu Rev Immunol, 17:593-623, 1999.

[168] N. Ninomiya, K. Hazeki, Y. Fukui, T. Seya, T. Okada, O. Hazeki, and M. Ui. Involvement of phosphatidylinositol 3-kinase in fc gamma receptor signaling. J Biol Chem, 269(36):22732-7, 1994. 
[169] N. Araki, M. T. Johnson, and J. A. Swanson. A role for phosphoinositide 3-kinase in the completion of macropinocytosis and phagocytosis by macrophages. J Cell Biol, 135(5):1249-60, 1996.

[170] D. Cox, P. Chang, Q. Zhang, P. G. Reddy, G. M. Bokoch, and S. Greenberg. Requirements for both rac1 and cdc42 in membrane ruffling and phagocytosis in leukocytes. J Exp Med, 186(9):1487-94, 1997.

[171] Q. Zhang, D. Cox, C. C. Tseng, J. G. Donaldson, and S. Greenberg. A requirement for arf6 in fcgamma receptor-mediated phagocytosis in macrophages. J Biol Chem, 273(32):19977-81, 1998.

[172] V. A. Fadok, J. S. Savill, C. Haslett, D. L. Bratton, D. E. Doherty, P. A. Campbell, and P. M. Henson. Different populations of macrophages use either the vitronectin receptor or the phosphatidylserine receptor to recognize and remove apoptotic cells. J Immunol, 149(12):4029-35, 1992.

[173] D. Pradhan, S. Krahling, P. Williamson, and R. A. Schlegel. Multiple systems for recognition of apoptotic lymphocytes by macrophages. Mol Biol Cell, 8(5):767-78, 1997.

[174] M. Kirkham and R. G. Parton. Clathrin-independent endocytosis: new insights into caveolae and non-caveolar lipid raft carriers. Biochim Biophys Acta, 1746(3):349-63, 2005 .

[175] J. Mercer and A. Helenius. Virus entry by macropinocytosis. Nat Cell Biol, 11(5):510-20, 2009.

[176] C. Watts and M. Marsh. Endocytosis: what goes in and how? J Cell Sci, 103 ( Pt 1):1-8, 1992.

[177] M. Watarai, I. Derre, J. Kirby, J. D. Growney, W. F. Dietrich, and R. R. Isberg. Legionella pneumophila is internalized by a macropinocytotic uptake pathway controlled by the dot/icm system and the mouse lgn1 locus. J Exp Med, 194(8):1081-96, 2001.

[178] C. L. Francis, T. A. Ryan, B. D. Jones, S. J. Smith, and S. Falkow. Ruffles induced by salmonella and other stimuli direct macropinocytosis of bacteria. Nature, 364(6438):639-42, 1993.

[179] A. Fabbri, L. Falzano, S. Travaglione, A. Stringaro, W. Malorni, S. Fais, and C. Fiorentini. Rho-activating escherichia coli cytotoxic necrotizing factor 1: 
macropinocytosis of apoptotic bodies in human epithelial cells. Int J Med Microbiol, 291(6-7):551-4, 2002.

[180] P. R. Hoffmann, A. M. deCathelineau, C. A. Ogden, Y. Leverrier, D. L. Bratton, D. L. Daleke, A. J. Ridley, V. A. Fadok, and P. M. Henson. Phosphatidylserine (ps) induces ps receptor-mediated macropinocytosis and promotes clearance of apoptotic cells. J Cell Biol, 155(4):649-59, 2001.

[181] M. Maniak. Conserved features of endocytosis in dictyostelium. Int Rev Cytol, 221:257-87, 2002.

[182] J. Cardelli. Phagocytosis and macropinocytosis in dictyostelium: phosphoinositidebased processes, biochemically distinct. Traffic, 2(5):311-20, 2001.

[183] E. L. Racoosin and J. A. Swanson. Macropinosome maturation and fusion with tubular lysosomes in macrophages. J Cell Biol, 121(5):1011-20, 1993.

[184] S. D. Xiang, A. Scholzen, G. Minigo, C. David, V. Apostolopoulos, P. L. Mottram, and M. Plebanski. Pathogen recognition and development of particulate vaccines: does size matter? Methods, 40(1):1-9, 2006.

[185] K. Inaba, M. Inaba, M. Naito, and R. M. Steinman. Dendritic cell progenitors phagocytose particulates, including bacillus calmette-guerin organisms, and sensitize mice to mycobacterial antigens in vivo. J Exp Med, 178(2):479-88, 1993.

[186] F. Sallusto, M. Cella, C. Danieli, and A. Lanzavecchia. Dendritic cells use macropinocytosis and the mannose receptor to concentrate macromolecules in the major histocompatibility complex class ii compartment: downregulation by cytokines and bacterial products. J Exp Med, 182(2):389-400, 1995.

[187] J. M. Austyn. New insights into the mobilization and phagocytic activity of dendritic cells. J Exp Med, 183(4):1287-92, 1996.

[188] W. S. Garrett, L. M. Chen, R. Kroschewski, M. Ebersold, S. Turley, S. Trombetta, J. E. Galan, and I. Mellman. Developmental control of endocytosis in dendritic cells by cdc42. Cell, 102(3):325-34, 2000.

[189] S. Kuhlmann-Krieg, I. Sommer, and M. Schachner. Ultrastructural features of cultured oligodendrocytes expressing stage-specific cell-surface antigens. Brain Res, 467(2):269-80, 1988.

[190] I. Sommer and M. Schachner. Monoclonal antibodies (o1 to o4) to oligodendrocyte cell surfaces: an immunocytological study in the central nervous system. Dev Biol, 83(2):311-27, 1981. 
[191] J. M. Greer, C. A. Dyer, M. Pakaski, C. Symonowicz, and M. B. Lees. Orientation of myelin proteolipid protein in the oligodendrocyte cell membrane. Neurochem Res, 21(4):431-40, 1996.

[192] C. Bourquin, A. Iglesias, T. Berger, H. Wekerle, and C. Linington. Myelin oligodendrocyte glycoprotein-dna vaccination induces antibody-mediated autoaggression in experimental autoimmune encephalomyelitis. Eur J Immunol, 30(12):3663-71, 2000 .

[193] K. Trajkovic, A. S. Dhaunchak, J. T. Goncalves, D. Wenzel, A. Schneider, G. Bunt, K. A. Nave, and M. Simons. Neuron to glia signaling triggers myelin membrane exocytosis from endosomal storage sites. J Cell Biol, 172(6):937-48, 2006.

[194] T.; Russel D.W. Sambrook, J.; Maniatis. Molecular cloning: a laboratory manual. Cold Spring Harbor Laboratory Press;, 3rd edition, 2001.

[195] M. Jung, E. Kramer, M. Grzenkowski, K. Tang, W. Blakemore, A. Aguzzi, K. Khazaie, K. Chlichlia, G. von Blankenfeld, H. Kettenmann, and et al. Lines of murine oligodendroglial precursor cells immortalized by an activated neu tyrosine kinase show distinct degrees of interaction with axons in vitro and in vivo. Eur $J$ Neurosci, $7(6): 1245-65,1995$.

[196] P. K. Smith, R. I. Krohn, G. T. Hermanson, A. K. Mallia, F. H. Gartner, M. D. Provenzano, E. K. Fujimoto, N. M. Goeke, B. J. Olson, and D. C. Klenk. Measurement of protein using bicinchoninic acid. Anal Biochem, 150(1):76-85, 1985.

[197] U. K. Laemmli. Cleavage of structural proteins during the assembly of the head of bacteriophage t4. Nature, 227(5259):680-5, 1970.

[198] H. Towbin, T. Staehelin, and J. Gordon. Electrophoretic transfer of proteins from polyacrylamide gels to nitrocellulose sheets: procedure and some applications. Proc Natl Acad Sci U S A, 76(9):4350-4, 1979.

[199] D. Fitzner, A. Schneider, A. Kippert, W. Mobius, K. I. Willig, S. W. Hell, G. Bunt, K. Gaus, and M. Simons. Myelin basic protein-dependent plasma membrane reorganization in the formation of myelin. EMBO J, 25(21):5037-48, 2006.

[200] P. L. Felgner, T. R. Gadek, M. Holm, R. Roman, H. W. Chan, M. Wenz, J. P. Northrop, G. M. Ringold, and M. Danielsen. Lipofection: a highly efficient, lipidmediated dna-transfection procedure. Proc Natl Acad Sci U S A, 84(21):7413-7, 1987. 
[201] A. E. Morelli, A. T. Larregina, W. J. Shufesky, M. L. Sullivan, D. B. Stolz, G. D. Papworth, A. F. Zahorchak, A. J. Logar, Z. Wang, S. C. Watkins, Jr. Falo, L. D., and A. W. Thomson. Endocytosis, intracellular sorting, and processing of exosomes by dendritic cells. Blood, 104(10):3257-66, 2004.

[202] P. K. Horan and S. E. Slezak. Stable cell membrane labelling. Nature, 340(6229):1678, 1989.

[203] T. Tian, Y. Wang, H. Wang, Z. Zhu, and Z. Xiao. Visualizing of the cellular uptake and intracellular trafficking of exosomes by live-cell microscopy. J Cell Biochem.

[204] B. Mui, L. Chow, and M. J. Hope. Extrusion technique to generate liposomes of defined size. Methods Enzymol, 367:3-14, 2003.

[205] W. T. Norton and S. E. Poduslo. Myelination in rat brain: method of myelin isolation. J Neurochem, 21(4):749-57, 1973.

[206] J. N. Larocca and W. T. Norton. Isolation of myelin. Curr Protoc Cell Biol, Chapter 3:Unit3 25, 2007.

[207] D. van Rossum, S. Hilbert, S. Strassenburg, U. K. Hanisch, and W. Bruck. Myelinphagocytosing macrophages in isolated sciatic and optic nerves reveal a unique reactive phenotype. Glia, 56(3):271-83, 2008.

[208] E. Bettelli, M. Pagany, H. L. Weiner, C. Linington, R. A. Sobel, and V. K. Kuchroo. Myelin oligodendrocyte glycoprotein-specific t cell receptor transgenic mice develop spontaneous autoimmune optic neuritis. J Exp Med, 197(9):1073-81, 2003.

[209] G. Krishnamoorthy, H. Lassmann, H. Wekerle, and A. Holz. Spontaneous opticospinal encephalomyelitis in a double-transgenic mouse model of autoimmune $t$ cell/b cell cooperation. J Clin Invest, 116(9):2385-92, 2006.

[210] M. Simons, E. M. Kramer, P. Macchi, S. Rathke-Hartlieb, J. Trotter, K. A. Nave, and J. B. Schulz. Overexpression of the myelin proteolipid protein leads to accumulation of cholesterol and proteolipid protein in endosomes/lysosomes: implications for pelizaeus-merzbacher disease. J Cell Biol, 157(2):327-36, 2002.

[211] W. Denk, J. H. Strickler, and W. W. Webb. Two-photon laser scanning fluorescence microscopy. Science, 248(4951):73-6, 1990.

[212] B. D. Minsky and A. M. Cohen. Minimizing the toxicity of pelvic radiation therapy in rectal cancer. Oncology (Williston Park), 2(8):21-5, 28-9, 1988.

[213] H. Hutter. Fluorescent reporter methods. Methods Mol Biol, 351:155-73, 2006. 
[214] S. Jung, J. Aliberti, P. Graemmel, M. J. Sunshine, G. W. Kreutzberg, A. Sher, and D. R. Littman. Analysis of fractalkine receptor cx $(3) \mathrm{cr} 1$ function by targeted deletion and green fluorescent protein reporter gene insertion. Mol Cell Biol, 20(11):4106-14, 2000 .

[215] E. Macia, M. Ehrlich, R. Massol, E. Boucrot, C. Brunner, and T. Kirchhausen. Dynasore, a cell-permeable inhibitor of dynamin. Dev Cell, 10(6):839-50, 2006.

[216] H. Akbar, J. Cancelas, D. A. Williams, J. Zheng, and Y. Zheng. Rational design and applications of a rac gtpase-specific small molecule inhibitor. Methods Enzymol, 406:554-65, 2006.

[217] D. W. Goddette and C. Frieden. Actin polymerization. the mechanism of action of cytochalasin d. J Biol Chem, 261(34):15974-80, 1986.

[218] M. Koivusalo, C. Welch, H. Hayashi, C. C. Scott, M. Kim, T. Alexander, N. Touret, K. M. Hahn, and S. Grinstein. Amiloride inhibits macropinocytosis by lowering submembranous ph and preventing rac1 and cdc42 signaling. J Cell Biol, 188(4):54763.

[219] S. Drose and K. Altendorf. Bafilomycins and concanamycins as inhibitors of vatpases and p-atpases. J Exp Biol, 200(Pt 1):1-8, 1997.

[220] M. Wu. Enhancement of immunotoxin activity using chemical and biological reagents. $\mathrm{Br} J$ Cancer, 75(9):1347-55, 1997.

[221] J. Mercer and A. Helenius. Vaccinia virus uses macropinocytosis and apoptotic mimicry to enter host cells. Science, 320(5875):531-5, 2008.

[222] J. Husemann, J. D. Loike, R. Anankov, M. Febbraio, and S. C. Silverstein. Scavenger receptors in neurobiology and neuropathology: their role on microglia and other cells of the nervous system. Glia, 40(2):195-205, 2002.

[223] J. Hughes, Y. Liu, J. Van Damme, and J. Savill. Human glomerular mesangial cell phagocytosis of apoptotic neutrophils: mediation by a novel cd36-independent vitronectin receptor/thrombospondin recognition mechanism that is uncoupled from chemokine secretion. J Immunol, 158(9):4389-97, 1997.

[224] K. S. Ravichandran and U. Lorenz. Engulfment of apoptotic cells: signals for a good meal. Nat Rev Immunol, 7(12):964-74, 2007. 
[225] A. F. Fomina, T. J. Deerinck, M. H. Ellisman, and M. D. Cahalan. Regulation of membrane trafficking and subcellular organization of endocytic compartments revealed with fm1-43 in resting and activated human t cells. Exp Cell Res, 291(1):15066, 2003.

[226] M. Miyanishi, K. Tada, M. Koike, Y. Uchiyama, T. Kitamura, and S. Nagata. Identification of tim4 as a phosphatidylserine receptor. Nature, 450(7168):435-9, 2007.

[227] R. Hanayama, M. Tanaka, K. Miwa, A. Shinohara, A. Iwamatsu, and S. Nagata. Identification of a factor that links apoptotic cells to phagocytes. Nature, 417(6885):182-7, 2002.

[228] F. Reichert and S. Rotshenker. Complement-receptor-3 and scavenger-receptorai/ii mediated myelin phagocytosis in microglia and macrophages. Neurobiol Dis, 12(1):65-72, 2003.

[229] T. A. Hamilton, Y. Ohmori, J. M. Tebo, and R. Kishore. Regulation of macrophage gene expression by pro- and anti-inflammatory cytokines. Pathobiology, 67(5-6):2414, 1999.

[230] C. A. Colton and D. M. Wilcock. Assessing activation states in microglia. CNS Neurol Disord Drug Targets, 9(2):174-91, 2010.

[231] B. Pollinger, G. Krishnamoorthy, K. Berer, H. Lassmann, M. R. Bosl, R. Dunn, H. S. Domingues, A. Holz, F. C. Kurschus, and H. Wekerle. Spontaneous relapsingremitting eae in the $\mathrm{sjl} / \mathrm{j}$ mouse: Mog-reactive transgenic $\mathrm{t}$ cells recruit endogenous mog-specific b cells. J Exp Med, 206(6):1303-16, 2009.

[232] C. M. Pelfrey, L. R. Tranquill, A. B. Vogt, and H. F. McFarland. T cell response to two immunodominant proteolipid protein (plp) peptides in multiple sclerosis patients and healthy controls. Mult Scler, 1(5):270-8, 1996.

[233] C. D. Schmid, L. N. Sautkulis, P. E. Danielson, J. Cooper, K. W. Hasel, B. S. Hilbush, J. G. Sutcliffe, and M. J. Carson. Heterogeneous expression of the triggering receptor expressed on myeloid cells-2 on adult murine microglia. J Neurochem, 83(6):1309-20, 2002.

[234] L. B. Moran, D. C. Duke, and M. B. Graeber. The microglial gene regulatory network activated by interferon-gamma. J Neuroimmunol, 183(1-2):1-6, 2007.

[235] S. Lund, K. V. Christensen, M. Hedtjarn, A. L. Mortensen, H. Hagberg, J. Falsig, H. Hasseldam, A. Schrattenholz, P. Porzgen, and M. Leist. The dynamics of the lps 
triggered inflammatory response of murine microglia under different culture and in vivo conditions. J Neuroimmunol, 180(1-2):71-87, 2006.

[236] J. M. Kinchen and K. S. Ravichandran. Journey to the grave: signaling events regulating removal of apoptotic cells. J Cell Sci, 120(Pt 13):2143-9, 2007.

[237] R. M. Steinman and J. Swanson. The endocytic activity of dendritic cells. J Exp Med, 182(2):283-8, 1995.

[238] A. J. Ridley, H. F. Paterson, C. L. Johnston, D. Diekmann, and A. Hall. The small gtp-binding protein rac regulates growth factor-induced membrane ruffling. Cell, 70(3):401-10, 1992.

[239] M. A. West, A. R. Prescott, E. L. Eskelinen, A. J. Ridley, and C. Watts. Rac is required for constitutive macropinocytosis by dendritic cells but does not control its downregulation. Curr Biol, 10(14):839-48, 2000.

[240] M. C. Kerr and R. D. Teasdale. Defining macropinocytosis. Traffic, 10(4):364-71, 2009 .

[241] O. I. Stendahl, J. H. Hartwig, E. A. Brotschi, and T. P. Stossel. Distribution of actinbinding protein and myosin in macrophages during spreading and phagocytosis. $J$ Cell Biol, 84(2):215-24, 1980.

[242] S. M. Sweitzer and J. E. Hinshaw. Dynamin undergoes a gtp-dependent conformational change causing vesiculation. Cell, 93(6):1021-9, 1998.

[243] D. Feng, W. L. Zhao, Y. Y. Ye, X. C. Bai, R. Q. Liu, L. F. Chang, Q. Zhou, and S. F. Sui. Cellular internalization of exosomes occurs through phagocytosis. Traffic, 11(5):675-87.

[244] J. F. Fortin, R. Cantin, and M. J. Tremblay. T cells expressing activated lfa-1 are more susceptible to infection with human immunodeficiency virus type 1 particles bearing host-encoded icam-1. J Virol, 72(3):2105-12, 1998.

[245] M. R. Tardif and M. J. Tremblay. Lfa-1 is a key determinant for preferential infection of memory cd $4+\mathrm{t}$ cells by human immunodeficiency virus type 1 . J Virol, 79(21):13714-24, 2005.

[246] S. Rieu, C. Geminard, H. Rabesandratana, J. Sainte-Marie, and M. Vidal. Exosomes released during reticulocyte maturation bind to fibronectin via integrin alpha4beta1. Eur J Biochem, 267(2):583-90, 2000. 
[247] A. Clayton, A. Turkes, S. Dewitt, R. Steadman, M. D. Mason, and M. B. Hallett. Adhesion and signaling by b cell-derived exosomes: the role of integrins. FASEB J, 18(9):977-9, 2004.

[248] C. Thery, L. Zitvogel, and S. Amigorena. Exosomes: composition, biogenesis and function. Nat Rev Immunol, 2(8):569-79, 2002.

[249] V. A. Fadok, D. L. Bratton, S. C. Frasch, M. L. Warner, and P. M. Henson. The role of phosphatidylserine in recognition of apoptotic cells by phagocytes. Cell Death Differ, 5(7):551-62, 1998.

[250] A. Witting, P. Muller, A. Herrmann, H. Kettenmann, and C. Nolte. Phagocytic clearance of apoptotic neurons by microglia/brain macrophages in vitro: involvement of lectin-, integrin-, and phosphatidylserine-mediated recognition. J Neurochem, 75(3):1060-70, 2000.

[251] P. Veron, E. Segura, G. Sugano, S. Amigorena, and C. Thery. Accumulation of mfge8/lactadherin on exosomes from immature dendritic cells. Blood Cells Mol Dis, $35(2): 81-8,2005$.

[252] D. Park, A. C. Tosello-Trampont, M. R. Elliott, M. J. Lu, L. B. Haney, Z. Ma, A. L. Klibanov, J. W. Mandell, and K. S. Ravichandran. Bai1 is an engulfment receptor for apoptotic cells upstream of the elmo/dock180/rac module. Nature, 450(7168):430-U10, 2007.

[253] S. J. Gardai, K. A. McPhillips, S. C. Frasch, W. J. Janssen, A. Starefeldt, J. E. Murphy-Ullrich, D. L. Bratton, P. A. Oldenborg, M. Michalak, and P. M. Henson. Cell-surface calreticulin initiates clearance of viable or apoptotic cells through transactivation of lrp on the phagocyte. Cell, 123(2):321-34, 2005.

[254] L. Zakharova, M. Svetlova, and A. F. Fomina. T cell exosomes induce cholesterol accumulation in human monocytes via phosphatidylserine receptor. J Cell Physiol, 212(1):174-81, 2007.

[255] K. Williams, E. Ulvestad, A. Waage, J. P. Antel, and J. McLaurin. Activation of adult human derived microglia by myelin phagocytosis in vitro. J Neurosci Res, 38(4):433-43, 1994.

[256] K. Mosley and M. L. Cuzner. Receptor-mediated phagocytosis of myelin by macrophages and microglia: effect of opsonization and receptor blocking agents. Neurochem Res, 21(4):481-7, 1996. 
[257] L. J. van der Laan, S. R. Ruuls, K. S. Weber, I. J. Lodder, E. A. Dopp, and C. D. Dijkstra. Macrophage phagocytosis of myelin in vitro determined by flow cytometry: phagocytosis is mediated by cr3 and induces production of tumor necrosis factoralpha and nitric oxide. J Neuroimmunol, 70(2):145-52, 1996.

[258] L. A. Boven, M. Van Meurs, M. Van Zwam, A. Wierenga-Wolf, R. Q. Hintzen, R. G. Boot, J. M. Aerts, S. Amor, E. E. Nieuwenhuis, and J. D. Laman. Myelin-laden macrophages are anti-inflammatory, consistent with foam cells in multiple sclerosis. Brain, 129(Pt 2):517-26, 2006.

[259] Y. Liu, W. Hao, M. Letiembre, S. Walter, M. Kulanga, H. Neumann, and K. Fassbender. Suppression of microglial inflammatory activity by myelin phagocytosis: role of p47-phox-mediated generation of reactive oxygen species. J Neurosci, 26(50):1290413, 2006.

[260] D. M. Muller, M. P. Pender, and J. M. Greer. Chemokines and chemokine receptors: potential therapeutic targets in multiple sclerosis. Curr Drug Targets Inflamm Allergy, 3(3):279-90, 2004.

[261] K. Biber, M. W. Zuurman, I. M. Dijkstra, and H. W. Boddeke. Chemokines in the brain: neuroimmunology and beyond. Curr Opin Pharmacol, 2(1):63-8, 2002.

[262] P. Rezaie and D. Male. Colonisation of the developing human brain and spinal cord by microglia: a review. Microsc Res Tech, 45(6):359-82, 1999.

[263] C. A. Dinarello, R. A. Dempsey, M. Allegretta, G. LoPreste, N. Dainiak, D. R. Parkinson, and J. W. Mier. Inhibitory effects of elevated temperature on human cytokine production and natural killer activity. Cancer Res, 46(12 Pt 1):6236-41, 1986.

[264] J. Mercer, M. Schelhaas, and A. Helenius. Virus entry by endocytosis. Annu Rev Biochem, 79:803-33.

[265] R. De Simone, A. Giampaolo, B. Giometto, P. Gallo, G. Levi, C. Peschle, and F. Aloisi. The costimulatory molecule b7 is expressed on human microglia in culture and in multiple sclerosis acute lesions. J Neuropathol Exp Neurol, 54(2):175-87, 1995 .

[266] T. Dutta, A. Spence, and L. A. Lampson. Robust ability of ifn-gamma to upregulate class ii mhc antigen expression in tumor bearing rat brains. $J$ Neurooncol, 64(1-2):3144, 2003. 
[267] A. S. Freedman, G. J. Freeman, K. Rhynhart, and L. M. Nadler. Selective induction of b7/bb-1 on interferon-gamma stimulated monocytes: a potential mechanism for amplification of $\mathrm{t}$ cell activation through the cd28 pathway. Cell Immunol, 137(2):429-37, 1991.

[268] K. S. Hathcock, G. Laszlo, C. Pucillo, P. Linsley, and R. J. Hodes. Comparative analysis of b7-1 and b7-2 costimulatory ligands: expression and function. $J$ Exp Med, 180(2):631-40, 1994.

[269] G. J. Freeman, V. A. Boussiotis, A. Anumanthan, G. M. Bernstein, X. Y. Ke, P. D. Rennert, G. S. Gray, J. G. Gribben, and L. M. Nadler. B7-1 and b7-2 do not deliver identical costimulatory signals, since b7-2 but not b7-1 preferentially costimulates the initial production of il-4. Immunity, 2(5):523-32, 1995.

[270] V. K. Kuchroo, M. P. Das, J. A. Brown, A. M. Ranger, S. S. Zamvil, R. A. Sobel, H. L. Weiner, N. Nabavi, and L. H. Glimcher. B7-1 and b7-2 costimulatory molecules activate differentially the th1/th2 developmental pathways: application to autoimmune disease therapy. Cell, 80(5):707-18, 1995.

[271] B. Perussia. Lymphokine-activated killer cells, natural killer cells and cytokines. Curr Opin Immunol, 3(1):49-55, 1991.

[272] T. R. Mosmann and R. L. Coffman. Th1 and th2 cells: different patterns of lymphokine secretion lead to different functional properties. Annu Rev Immunol, 7:14573, 1989 .

[273] S. Sad, R. Marcotte, and T. R. Mosmann. Cytokine-induced differentiation of precursor mouse cd8 $+\mathrm{t}$ cells into cytotoxic cd8 $+\mathrm{t}$ cells secreting th1 or th2 cytokines. Immunity, 2(3):271-9, 1995.

[274] B. G. Xiao, X. F. Bai, G. X. Zhang, B. Hojeberg, and H. Link. Shift from anti- to proinflammatory cytokine profiles in microglia through lps- or ifn-gamma-mediated pathways. Neuroreport, 7(12):1893-8, 1996.

[275] Y. Matsumoto, K. Ohmori, and M. Fujiwara. Immune regulation by brain cells in the central nervous system: microglia but not astrocytes present myelin basic protein to encephalitogenic t cells under in vivo-mimicking conditions. Immunology, 76(2):209-16, 1992.

[276] D. K. Dalton, S. Pitts-Meek, S. Keshav, I. S. Figari, A. Bradley, and T. A. Stewart. Multiple defects of immune cell function in mice with disrupted interferon-gamma genes. Science, 259(5102):1739-42, 1993. 
[277] S. Huang, W. Hendriks, A. Althage, S. Hemmi, H. Bluethmann, R. Kamijo, J. Vilcek, R. M. Zinkernagel, and M. Aguet. Immune response in mice that lack the interferon-gamma receptor. Science, 259(5102):1742-5, 1993.

[278] M. A. Meraz, J. M. White, K. C. Sheehan, E. A. Bach, S. J. Rodig, A. S. Dighe, D. H. Kaplan, J. K. Riley, A. C. Greenlund, D. Campbell, K. Carver-Moore, R. N. DuBois, R. Clark, M. Aguet, and R. D. Schreiber. Targeted disruption of the stat1 gene in mice reveals unexpected physiologic specificity in the jak-stat signaling pathway. Cell, 84(3):431-42, 1996.

[279] J. E. Durbin, R. Hackenmiller, M. C. Simon, and D. E. Levy. Targeted disruption of the mouse stat1 gene results in compromised innate immunity to viral disease. Cell, 84(3):443-50, 1996.

[280] C. V. Harding and H. J. Geuze. Class ii mhc molecules are present in macrophage lysosomes and phagolysosomes that function in the phagocytic processing of listeria monocytogenes for presentation to t cells. J Cell Biol, 119(3):531-42, 1992.

[281] H. Geuze. Ejcb-lecture. a novel lysosomal compartment engaged in antigen presentation. Eur J Cell Biol, 64(1):3-6, 1994.

[282] M. P. Peppelenbosch, M. DeSmedt, G. Pynaert, S. J. van Deventer, and J. Grooten. Macrophages present pinocytosed exogenous antigen via mhc class i whereas antigen ingested by receptor-mediated endocytosis is presented via mhe class ii. J Immunol, 165(4):1984-91, 2000.

[283] C. Beauvillain, S. Donnou, U. Jarry, M. Scotet, H. Gascan, Y. Delneste, P. Guermonprez, P. Jeannin, and D. Couez. Neonatal and adult microglia cross-present exogenous antigens. Glia, 56(1):69-77, 2008.

[284] L. Delamarre, M. Pack, H. Chang, I. Mellman, and E. S. Trombetta. Differential lysosomal proteolysis in antigen-presenting cells determines antigen fate. Science, 307(5715):1630-4, 2005.

[285] S. Burgdorf and C. Kurts. Endocytosis mechanisms and the cell biology of antigen presentation. Curr Opin Immunol, 20(1):89-95, 2008.

[286] S. A. Porcelli and R. L. Modlin. The cd1 system: antigen-presenting molecules for t cell recognition of lipids and glycolipids. Annu Rev Immunol, 17:297-329, 1999.

[287] D. C. Barral and M. B. Brenner. Cd1 antigen presentation: how it works. Nat Rev Immunol, 7(12):929-41, 2007. 
[288] P. A. Sieling, D. Chatterjee, S. A. Porcelli, T. I. Prigozy, R. J. Mazzaccaro, T. Soriano, B. R. Bloom, M. B. Brenner, M. Kronenberg, P. J. Brennan, and et al. Cd1restricted t cell recognition of microbial lipoglycan antigens. Science, 269(5221):22730, 1995.

[289] T. Kawano, J. Cui, Y. Koezuka, I. Toura, Y. Kaneko, K. Motoki, H. Ueno, R. Nakagawa, H. Sato, E. Kondo, H. Koseki, and M. Taniguchi. Cd1d-restricted and tcr-mediated activation of valpha14 nkt cells by glycosylceramides. Science, 278(5343):1626-9, 1997.

[290] M. Koch, V. S. Stronge, D. Shepherd, S. D. Gadola, B. Mathew, G. Ritter, A. R. Fersht, G. S. Besra, R. R. Schmidt, E. Y. Jones, and V. Cerundolo. The crystal structure of human cd1d with and without alpha-galactosylceramide. Nat Immunol, 6(8):819-26, 2005.

[291] S. Joyce, A. S. Woods, J. W. Yewdell, J. R. Bennink, A. D. De Silva, A. Boesteanu, S. P. Balk, R. J. Cotter, and R. R. Brutkiewicz. Natural ligand of mouse cd1d1: cellular glycosylphosphatidylinositol. Science, 279(5356):1541-4, 1998.

[292] A. Shamshiev, H. J. Gober, A. Donda, Z. Mazorra, L. Mori, and G. De Libero. Presentation of the same glycolipid by different cd1 molecules. J Exp Med, 195(8):101321, 2002.

[293] G. De Libero and L. Mori. How the immune system detects lipid antigens. Prog Lipid Res, 49(2):120-7.

[294] A. Shamshiev, A. Donda, T. I. Prigozy, L. Mori, V. Chigorno, C. A. Benedict, L. Kappos, S. Sonnino, M. Kronenberg, and G. De Libero. The alphabeta t cell response to self-glycolipids shows a novel mechanism of cd1b loading and a requirement for complex oligosaccharides. Immunity, 13(2):255-64, 2000.

[295] A. Shamshiev, A. Donda, I. Carena, L. Mori, L. Kappos, and G. De Libero. Self glycolipids as t-cell autoantigens. Eur J Immunol, 29(5):1667-75, 1999.

[296] M. Amyere, M. Mettlen, P. Van Der Smissen, A. Platek, B. Payrastre, A. Veithen, and P. J. Courtoy. Origin, originality, functions, subversions and molecular signalling of macropinocytosis. Int J Med Microbiol, 291(6-7):487-94, 2002.

[297] P. Dowrick, P. Kenworthy, B. McCann, and R. Warn. Circular ruffle formation and closure lead to macropinocytosis in hepatocyte growth factor/scatter factor-treated cells. Eur J Cell Biol, 61(1):44-53, 1993. 
[298] L. J. Hewlett, A. R. Prescott, and C. Watts. The coated pit and macropinocytic pathways serve distinct endosome populations. J Cell Biol, 124(5):689-703, 1994.

[299] M. Cella, A. Engering, V. Pinet, J. Pieters, and A. Lanzavecchia. Inflammatory stimuli induce accumulation of mhc class ii complexes on dendritic cells. Nature, 388(6644):782-7, 1997.

[300] I. Mellman, S. J. Turley, and R. M. Steinman. Antigen processing for amateurs and professionals. Trends Cell Biol, 8(6):231-7, 1998.

[301] R. M. Yates, A. Hermetter, G. A. Taylor, and D. G. Russell. Macrophage activation downregulates the degradative capacity of the phagosome. Traffic, 8(3):241-50, 2007.

[302] I. Jutras, M. Houde, N. Currier, J. Boulais, S. Duclos, S. LaBoissiere, E. Bonneil, P. Kearney, P. Thibault, E. Paramithiotis, P. Hugo, and M. Desjardins. Modulation of the phagosome proteome by interferon-gamma. Mol Cell Proteomics, 7(4):697$715,2008$.

[303] E. B. Pedersen, J. A. McNulty, A. J. Castro, L. M. Fox, J. Zimmer, and B. Finsen. Enriched immune-environment of blood-brain barrier deficient areas of normal adult rats. J Neuroimmunol, 76(1-2):117-31, 1997.

[304] L. P. McCluskey and L. A. Lampson. Local immune regulation in the central nervous system by substance p vs. glutamate. J Neuroimmunol, 116(2):136-46, 2001.

[305] N. A. Flaris, T. L. Densmore, M. C. Molleston, and W. F. Hickey. Characterization of microglia and macrophages in the central nervous system of rats: definition of the differential expression of molecules using standard and novel monoclonal antibodies in normal cns and in four models of parenchymal reaction. Glia, 7(1):34-40, 1993.

[306] A. C. Anderson, D. E. Anderson, L. Bregoli, W. D. Hastings, N. Kassam, C. Lei, R. Chandwaskar, J. Karman, E. W. Su, M. Hirashima, J. N. Bruce, L. P. Kane, V. K. Kuchroo, and D. A. Hafler. Promotion of tissue inflammation by the immune receptor tim-3 expressed on innate immune cells. Science, 318(5853):1141-3, 2007.

[307] S. Elkabes, E. M. DiCicco-Bloom, and I. B. Black. Brain microglia/macrophages express neurotrophins that selectively regulate microglial proliferation and function. J Neurosci, 16(8):2508-21, 1996.

[308] L. B. Moran, D. C. Duke, F. E. Turkheimer, R. B. Banati, and M. B. Graeber. Towards a transcriptome definition of microglial cells. Neurogenetics, 5(2):95-108, 2004 . 
[309] C. D. Schmid, B. Melchior, K. Masek, S. S. Puntambekar, P. E. Danielson, D. D. Lo, J. G. Sutcliffe, and M. J. Carson. Differential gene expression in lps/ifngamma activated microglia and macrophages: in vitro versus in vivo. $J$ Neurochem, 109 Suppl 1:117-25, 2009.

[310] K. Takahashi, M. Prinz, M. Stagi, O. Chechneva, and H. Neumann. Trem2transduced myeloid precursors mediate nervous tissue debris clearance and facilitate recovery in an animal model of multiple sclerosis. PLoS Med, 4(4):e124, 2007.

[311] I. R. Turnbull, S. Gilfillan, M. Cella, T. Aoshi, M. Miller, L. Piccio, M. Hernandez, and M. Colonna. Cutting edge: Trem-2 attenuates macrophage activation. $J$ Immunol, 177(6):3520-4, 2006.

[312] J. Paloneva, J. Mandelin, A. Kiialainen, T. Bohling, J. Prudlo, P. Hakola, M. Haltia, Y. T. Konttinen, and L. Peltonen. Dap12/trem2 deficiency results in impaired osteoclast differentiation and osteoporotic features. J Exp Med, 198(4):669-75, 2003.

[313] A. Bouchon, C. Hernandez-Munain, M. Cella, and M. Colonna. A dap12-mediated pathway regulates expression of cc chemokine receptor 7 and maturation of human dendritic cells. J Exp Med, 194(8):1111-22, 2001.

[314] G. J. Wright, M. J. Puklavec, A. C. Willis, R. M. Hoek, J. D. Sedgwick, M. H. Brown, and A. N. Barclay. Lymphoid/neuronal cell surface ox2 glycoprotein recognizes a novel receptor on macrophages implicated in the control of their function. Immunity, $13(2): 233-42,2000$.

[315] R. M. Hoek, S. R. Ruuls, C. A. Murphy, G. J. Wright, R. Goddard, S. M. Zurawski, B. Blom, M. E. Homola, W. J. Streit, M. H. Brown, A. N. Barclay, and J. D. Sedgwick. Down-regulation of the macrophage lineage through interaction with ox2 (cd200). Science, 290(5497):1768-71, 2000.

[316] H. Neumann. Control of glial immune function by neurons. Glia, 36(2):191-9, 2001.

[317] H. Neumann, T. Misgeld, K. Matsumuro, and H. Wekerle. Neurotrophins inhibit major histocompatibility class ii inducibility of microglia: involvement of the p75 neurotrophin receptor. Proc Natl Acad Sci U S A, 95(10):5779-84, 1998.

[318] D. B. Constam, J. Philipp, U. V. Malipiero, P. ten Dijke, M. Schachner, and A. Fontana. Differential expression of transforming growth factor-beta 1, -beta 2, and -beta 3 by glioblastoma cells, astrocytes, and microglia. J Immunol, 148(5):1404-10, 1992. 
[319] R. Wei and G. M. Jonakait. Neurotrophins and the anti-inflammatory agents interleukin-4 (il-4), il-10, il-11 and transforming growth factor-beta1 (tgf-beta1) down-regulate $\mathrm{t}$ cell costimulatory molecules b7 and cd40 on cultured rat microglia. J Neuroimmunol, 95(1-2):8-18, 1999.

[320] G. M. O'Keefe, V. T. Nguyen, and E. N. Benveniste. Class ii transactivator and class ii mhc gene expression in microglia: modulation by the cytokines tgf-beta, il-4, il-13 and il-10. Eur J Immunol, 29(4):1275-85, 1999.

[321] A. Rubartelli and M. T. Lotze. Inside, outside, upside down: damage-associated molecular-pattern molecules (damps) and redox. Trends Immunol, 28(10):429-36, 2007.

[322] D. M. Mosser. The many faces of macrophage activation. J Leukoc Biol, 73(2):209$12,2003$.

[323] S. Gordon. Alternative activation of macrophages. Nat Rev Immunol, 3(1):23-35, 2003.

[324] F. O. Martinez, A. Sica, A. Mantovani, and M. Locati. Macrophage activation and polarization. Front Biosci, 13:453-61, 2008.

[325] S. Gordon and P. R. Taylor. Monocyte and macrophage heterogeneity. Nat Rev Immunol, 5(12):953-64, 2005.

[326] R. M. Johnstone, M. Adam, J. R. Hammond, L. Orr, and C. Turbide. Vesicle formation during reticulocyte maturation. association of plasma membrane activities with released vesicles (exosomes). J Biol Chem, 262(19):9412-20, 1987.

[327] A. M. Booth, Y. Fang, J. K. Fallon, J. M. Yang, J. E. Hildreth, and S. J. Gould. Exosomes and hiv gag bud from endosome-like domains of the t cell plasma membrane. J Cell Biol, 172(6):923-35, 2006.

[328] V. Gieselmann, S. Franken, D. Klein, J. E. Mansson, R. Sandhoff, R. Lullmann Rauch, D. Hartmann, V. P. Saravanan, P. P. De Deyn, R. D'Hooge, A. M. Van Der Linden, and N. Schaeren-Wiemers. Metachromatic leukodystrophy: consequences of sulphatide accumulation. Acta Paediatr Suppl, 92(443):74-9; discussion 45, 2003.

[329] D. Dumont, J. P. Noben, M. Moreels, J. Vanderlocht, N. Hellings, F. Vandenabeele, I. Lambrichts, P. Stinissen, and J. Robben. Characterization of mature rat oligodendrocytes: a proteomic approach. J Neurochem, 102(2):562-76, 2007. 
[330] M. Bakhti, C. Winter, and M. Simons. Inhibition of myelin membrane sheath formation by oligodendrocyte-derived exosome-like vesicles. J Biol Chem.

[331] A. R. Taylor, M. B. Robinson, D. J. Gifondorwa, M. Tytell, and C. E. Milligan. Regulation of heat shock protein 70 release in astrocytes: role of signaling kinases. Dev Neurobiol, 67(13):1815-29, 2007.

[332] R. S. Fujinami, M. G. von Herrath, U. Christen, and J. L. Whitton. Molecular mimicry, bystander activation, or viral persistence: infections and autoimmune disease. Clin Microbiol Rev, 19(1):80-94, 2006.

[333] R. Hanayama, M. Tanaka, K. Miyasaka, K. Aozasa, M. Koike, Y. Uchiyama, and S. Nagata. Autoimmune disease and impaired uptake of apoptotic cells in mfg-e8deficient mice. Science, 304(5674):1147-50, 2004.

[334] A. Devitt, K. G. Parker, C. A. Ogden, C. Oldreive, M. F. Clay, L. A. Melville, C. O. Bellamy, A. Lacy-Hulbert, S. C. Gangloff, S. M. Goyert, and C. D. Gregory. Persistence of apoptotic cells without autoimmune disease or inflammation in cd14/- mice. J Cell Biol, 167(6):1161-70, 2004.

[335] M. Lucas, L. M. Stuart, A. Zhang, K. Hodivala-Dilke, M. Febbraio, R. Silverstein, J. Savill, and A. Lacy-Hulbert. Requirements for apoptotic cell contact in regulation of macrophage responses. J Immunol, 177(6):4047-54, 2006.

[336] R. S. Fujinami and M. B. Oldstone. Amino acid homology between the encephalitogenic site of myelin basic protein and virus: mechanism for autoimmunity. Science, 230(4729):1043-5, 1985.

[337] S. P. Deshpande, S. Lee, M. Zheng, B. Song, D. Knipe, J. A. Kapp, and B. T. Rouse. Herpes simplex virus-induced keratitis: evaluation of the role of molecular mimicry in lesion pathogenesis. J Virol, 75(7):3077-88, 2001.

[338] W. L. Beatty, H. J. Ullrich, and D. G. Russell. Mycobacterial surface moieties are released from infected macrophages by a constitutive exocytic event. Eur J Cell Biol, 80(1):31-40, 2001.

[339] J. D. Walker, C. L. Maier, and J. S. Pober. Cytomegalovirus-infected human endothelial cells can stimulate allogeneic cd4+ memory t cells by releasing antigenic exosomes. J Immunol, 182(3):1548-59, 2009.

[340] S. J. Gould, A. M. Booth, and J. E. Hildreth. The trojan exosome hypothesis. Proc Natl Acad Sci U S A, 100(19):10592-7, 2003. 
[341] D. G. Nguyen, A. Booth, S. J. Gould, and J. E. Hildreth. Evidence that hiv budding in primary macrophages occurs through the exosome release pathway. J Biol Chem, 278(52):52347-54, 2003.

[342] M. Jouve, N. Sol-Foulon, S. Watson, O. Schwartz, and P. Benaroch. Hiv-1 buds and accumulates in "nonacidic" endosomes of macrophages. Cell Host Microbe, $2(2): 85-95,2007$.

[343] S. Schmidt. Candidate autoantigens in multiple sclerosis. Mult Scler, 5(3):147-60, 1999. 


\section{Curriculum vitae}

Mareike Schnaars

born $20^{\text {th }}$ June 1981 in Bremen, Germany

Schildweg 38, 37085 Göttingen

E-Mail schnaars@em.mpg.de

Education and Research Experience

2008 - present Max Planck Institute of Experimental Medicine, Göttingen, Cellular Neuroscience, $\mathrm{PhD}$ thesis: Selective transfer of exosomes from oligodendrocytes to microglia by macropinocytosis

2008 - present Georg-August-University, Göttingen, International PhD Molecular Medicine program (Direct admission to $\mathrm{PhD}$ )

2006 - 2007 Max Planck Institute for Molecular Genetics, Berlin, Diploma Thesis

2004 - 2005 Faculte des Sciences, Marseille, France "Biologie des Eukaryotes"

2001 - 2007 University Bremen, Diploma studies in Biology

$2000 \quad$ Altes Gymnasium, Bremen, Germany: Abitur (A-Level)

Stipends \& Prizes

2010 Poster Prize at the PhD symposium "Protein Trafficking in Health and Disease"

$2004-2005 \quad$ Stipend for European Students (Erasmus program) 